\title{
Residual Overturning Circulation and Its Connection to Southern Ocean Dynamics
}

\author{
by \\ Madeleine Kendall Youngs \\ B.S., California Institute of Technology (2015) \\ Submitted to the Department of Earth, Atmospheric, and Planetary Sciences \\ in partial fulfillment of the requirements for the degree of \\ Doctor of Philosophy \\ at the \\ MASSACHUSETTS INSTITUTE OF TECHNOLOGY \\ and the \\ WOODS HOLE OCEANOGRAPHIC INSTITUTION
}

September 2020

(C)2020 Madeleine K. Youngs.

All rights reserved.

The author hereby grants to MIT and WHOI permission to reproduce and to distribute publicly paper and electronic copies of this thesis document in whole or in part in any medium now known or hereafter created.

Author

Department of Earth, Atmospheric and Planetary Sciences Massachusetts Institute of Technology \& Woods Hole Oceanographic Institution

August 19, 2020

Certified by . . .

Glenn R. Flierl

Professor of Oceanography Massachusetts Institute of Technology

Thesis Supervisor

Accepted by . .

Glenn Flierl

Chairman, Joint Committee for Physical Oceanography Massachusetts Institute of Technology Woods Hole Oceanographic Institution 


\title{
Residual Overturning Circulation and Its Connection to Southern Ocean Dynamics
}

\author{
by \\ Madeleine Kendall Youngs \\ Submitted to the Department of Earth, Atmospheric and Planetary Sciences
Massachusetts Institute of Technology
\& Woods Hole Oceanographic Institution
on August 19, 2020, in partial fulfillment of the
requirements for the degree of
Doctor of Philosophy
}

\begin{abstract}
Over the last 20 years, our understanding of the meridional overturning circulation has improved, but primarily in a two-dimensional, zonally-averaged framework. In this thesis, I have pushed beyond this simplification and shown that the additional complexity of meanders, storm tracks, and other zonal asymmetries is necessary to reproduce the lowest-order behavior of the overturning circulation. First I examined the role of basin width for determining whether the Atlantic or Pacific oceans experience deep convection. I used a two layered model and a rectangular single-basin model to show that the basin width, in combination with scalings for the overturning circulation make the overturning relatively weaker in the wider basin, priming it for a convection shut down.

In addition to this large-scale work, I have examined Southern Ocean-like meanders using a hierarchy of idealized models to understand the role of bottom topography in determining how the large-scale circulation responds to climate change scenarios. These are useful because they preserve the lowest-order behavior, while remaining simple enough to understand. I tested the response of the stratification and transport in the Southern Ocean to changes in wind using a highly-idealized two-layer quasi-geostrophic model. In addition to showing that meanders are necessary to reproduce the behavior of the Southern Ocean, I found that strong winds concentrate the baroclinic and barotropic instabilities downstream of the bottom topography and weaken the instabilities elsewhere due to a form-drag process. With weak winds, however, the system is essentially symmetric in longitude, like a flat-bottomed ocean. This result is consistent with observations of elevated turbulence downstream of major topography in the Southern Ocean. My next study investigated a more realistic Southern Ocean-like channel, with and without bottom topography, and examined the three-dimensional circulation in order to understand where vertical transport occurs and develop a picture of the pathways taken by each individual water parcel. I found that the vertical transport happens in very isolated locations, just downstream of topography. Finally, I added a biogeochemical model to my simulations and found that carbon fluxes are enhanced near topography, again highlighting the role of zonal asymmetries.
\end{abstract}

Thesis Supervisor: Glenn R. Flierl

Title: Professor of Oceanography

Massachusetts Institute of Technology 


\section{Acknowledgments}

I would like to acknowledge my funding sources and my committee and all the people I have had useful conversations with. I have been funded by the American Meteorological Society's Graduate Fellowship, as well as the National Defense Science and Engineering Graduate Fellowship. I have also been supported by NSF OCE-1536515 and NCAR Large Scale Computing Award UMIT0025.

I am grateful for my thesis committee, David Marshall, Nikki Lovenduski, and Mike Spall and my defense chair Irina Rypina. I am also grateful for useful discussions with Andrew Stewart, Mara Freilich, Jean-Michel Campin, Jon Lauderdale and many more. None of this would have been possible without my advisor, Glenn Flierl. I am very grateful for the advice

of Andrew Thompson, Greg Johnson, and Joe Pedlosky while carrying out other research projects. 


\section{Contents}

\begin{tabular}{lll}
\hline & Introduction & 23
\end{tabular}

1.1 Northern Deep Convection . . . . . . . . . . . . . . . . . . . . . 25

1.2 Southern Ocean Overturning $\ldots \ldots \ldots \ldots$

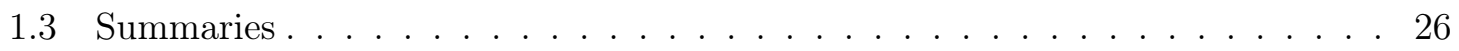

1.3.1 Basin-Width Dependence of Northern Deep Convection . . . . . . . . 26

1.3.2 Role of Residual Overturning for the Sensitivity of Southern Ocean Isopycnal Slopes to Changes in Wind Forcing . . . . . . . . . . . . . . 27

1.3.3 Causes of Localized Upwelling in a Southern Ocean-like Channel Near Topography . . . . . . . . . . . . . . . . . . . . . . . . 28

1.3.4 Localized Air-sea Carbon Fluxes due to Topography in a Southern Ocean-like Channel . . . . . . . . . . . . . . . . . . . . . . . . . . 28

1.3 .5 Conclusion $\ldots \ldots \ldots \ldots \ldots \ldots \ldots$

2 Basin-Width Dependence of Northern Deep Convection 31

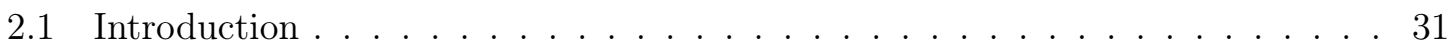

2.2 Two-layer Analytical Model $\ldots \ldots \ldots$. . . . . . . . . . . . . . . . . 32

$2.2 .1 \quad$ Scalings for Overturning . . . . . . . . . . . . . . . . . 34

$2.2 .2 \quad$ Salt Budget $\ldots \ldots \ldots \ldots \ldots$

$2.2 .3 \quad$ Overturning Shut-off $\ldots \ldots \ldots$. . . . . . . . . . . . . . 36

2.2 .4 Adiabatic limit . . . . . . . . . . . . . . . . . . . . . 37

2.3 Primitive Equations Model $\ldots \ldots \ldots \ldots$

$2.3 .1 \quad$ Configuration $\ldots \ldots \ldots \ldots \ldots$

2.3 .2 Results $\ldots \ldots \ldots \ldots$. . . . . . . . . . . . . . . . . . . . . . . . . . 40

2.4 Discussion and Conclusions $\ldots \ldots \ldots \ldots$. . . . . . . . . . . . 42 
3 Role of Residual Overturning for the Sensitivity of Southern Ocean Isopycnal Slopes to Changes in Wind Forcing $\quad 45$

3.1 Introduction . . . . . . . . . . . . . . . . . . . . . . . . . . . . . . . . . 45

3.2 A 2-Layer Quasi-Geostrophic Analogue of the Southern Ocean . . . . . . . . . 49

$3.2 .1 \quad$ Model Configuration $\ldots \ldots \ldots$. . . . . . . . . . . . . . . . . . 49

$3.2 .2 \quad$ Connection between overturning and slope . . . . . . . . . . . . . . 54

$3.2 .3 \quad$ Budgets . . . . . . . . . . . . . . . . . . . . . . . . . 55

3.3 Results . . . . . . . . . . . . . . . . . . . . . . . . . . . 57

$3.3 .1 \quad$ Strong wind forcing $\ldots \ldots \ldots \ldots$. . . . . . . . . . . . . 58

3.3 .2 Realistic Winds . . . . . . . . . . . . . . . . . . . . . . . . . 59

3.3 .3 No-wind Limit $\ldots \ldots \ldots$. . . . . . . . . . . . . . . . . . . . . . . . . . 62

3.3 .4 Connecting the Limits . . . . . . . . . . . . . . . . . 63

3.4 Discussion and Conclusions $\ldots \ldots \ldots \ldots$. . . . . . . . . . . . 64

4 Causes of Localized Upwelling in a Southern Ocean-like Channel Near $\begin{array}{ll}\text { Topography } & 67\end{array}$

4.1 Introduction . . . . . . . . . . . . . . . . . . . . . . . . 67

4.2 Numerical Model $\ldots \ldots \ldots$. . . . . . . . . . . . . . . . . . . . . . . . . . . . . . . . . . 68

4.2 .1 Boundary Conditions . . . . . . . . . . . . . . . . 70

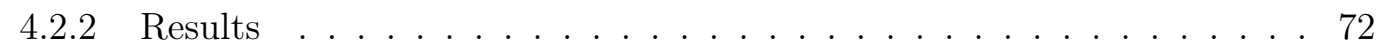

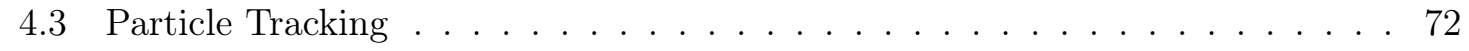

4.3 .1 Alternate Particle Tracking Method . . . . . . . . . . . . . . . 74

4.3 .2 Validation . . . . . . . . . . . . . . . . . . . . . . . . . . . . 74

4.4 Results $\ldots \ldots \ldots \ldots \ldots$

$4.4 .1 \quad$ Vertical Particle Motion $\ldots \ldots \ldots$. . . . . . . . . . . . . . 80

4.4 .2 Mean Flow . . . . . . . . . . . . . . . . . . . . . . . . . . . . . . . . 81

4.4 .3 Comparison to Flat-bottomed Ocean . . . . . . . . . . . . . . . . . . . 83

4.4 .4 Changes with Wind $\ldots \ldots \ldots \ldots$. . . . . . . . . . . . 84

4.5 Discussion and Conclusions $\ldots \ldots \ldots \ldots$. . . . . . . . . . . . . . . . 84

5 Localized Air-sea Carbon Fluxes due to Topography in a Southern Ocean$\begin{array}{ll}\text { like Channel } & 91\end{array}$

5.1 Introduction $\ldots \ldots \ldots \ldots \ldots \ldots \ldots$ 
5.2 Methods . . . . . . . . . . . . . . . . . . . . . . . 92

5.3 Analysis of Mean State . . . . . . . . . . . . . . . . . . . . . 93

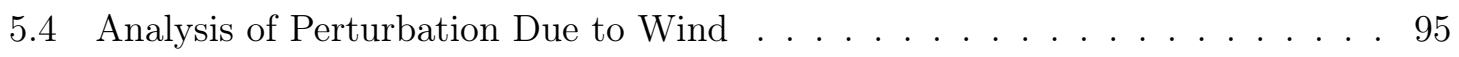

5.5 Discussion and Conclusions $\ldots \ldots \ldots \ldots \ldots \ldots$

$\begin{array}{lll}6 \text { Conclusions } & 99\end{array}$

6.1 Place of this thesis in the literature . . . . . . . . . . . . . . . . . . . . 99

$6.1 .1 \quad$ Basin-Width Dependence of Northern Deep Convection . . . . . . . . 99

6.1.2 Role of Residual Overturning for the Sensitivity of Southern Ocean Isopycnal Slopes to Changes in Wind Forcing . . . . . . . . . . . 100

6.1 .3 Causes of Localized Upwelling in a Southern Ocean-like Channel Near Topography . . . . . . . . . . . . . . . . . . . . . . . 100

6.1.4 Localized Air-sea Carbon Fluxes due to Topography in a Southern Ocean-like Channel . . . . . . . . . . . . . . . . . . . . . . . . . . . 101

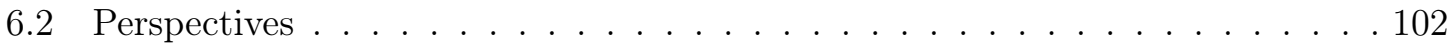

6.2 .1 Important Questions . . . . . . . . . . . . . . . . . . . . . . 102

6.2 .2 Challenges Facing the Community . . . . . . . . . . . . . 103

6.2 .3 Research Tools to Develop . . . . . . . . . . . . . . . . . . . . 104

\begin{tabular}{|ll}
\hline A Model Configuration for Chapter 2 & 107
\end{tabular}

B ACC Meanders, Energy Transfer and Mixed Barotropic-Baroclinic Insta$\begin{array}{ll}\text { bility } & 109\end{array}$

B.1 Introduction . . . . . . . . . . . . . . . . . . . . . . . . . . . . . . 109

B.2 Methods . . . . . . . . . . . . . . . . . . . . . . . . . . . . . . . . . . . . 112

B.2.1 Energy Conversion . . . . . . . . . . . . . . . . . . . . . . 112

B.2.2 Eddy Geometry . . . . . . . . . . . . . . . . . . . . . . . . 115

B.2.3 Model . . . . . . . . . . . . . . . . . . . . . . . . . . . . . 117

B.3 Energy Conversion . . . . . . . . . . . . . . . . . . . . . . 118

B.4 Geometric Analysis . . . . . . . . . . . . . . . . . . . . . 120

B.5 Discussion . . . . . . . . . . . . . . . . . . . . . . . 122

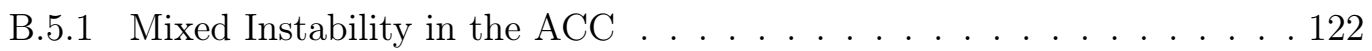

B.5.2 Meander Life Cycle. . . . . . . . . . . . . . . . . . . . . . 123 
B.6 Conclusions . . . . . . . . . . . . . . . . . . . . . . . . . 125 


\section{List of Figures}

1-1 This figure shows the conveyor belt circulation from Marshall and Speer 2012.

The colors represent different temperatures of water. The primary locations of sinking water are the North Atlantic and the Weddell and Ross Seas. The primary location of rising water is in the Southern Ocean. These are not actual trajectories of water because variability is significant in the actual movement of water parcels. . . . . . . . . . . . . . . . . . . . 24

1-2 This figure schematically shows our understanding of the overturning circulation. The wind blows over the Southern Ocean-like channel inducing a clockwise mean circulation, tilting up isopycnals. The eddy driven counterclockwise circulation acts to flatten isopycnals, leading to the residual circulation resulting from the partial cancellation of the components. Figure from Marshall and Speer $\mid[2012 \mid . \ldots \ldots \ldots$. . . . . . . . . . . . . 27

2-1 (a) Schematic of the conceptual model configuration. The ocean is divided in two layers of uniform temperature and salinity: a thermocline layer and a deep layer. A freshwater flux of $\mathcal{P} \mathrm{m}^{3} \mathrm{~s}^{-1}$ reduces salinity in the deep layer and an equal and opposite freshwater flux increases salinity in the thermocline layer, representing the dominance of precipitation at high latitudes and of evaporation at low latitudes. There are transports between the two layers: $\psi_{n}$ represents convection which converts light to dense water, $\psi_{u}$ represents the reverse conversion through deep ocean mixing and $\psi_{s}$ through meridional surface flows in a "Southern Ocean". (b) $\psi_{n}$ as a function of freshwater forcing, with the solid lines as the stable solutions, and dashed lines as the unstable solutions. (c) The difference in salinity between the deep and the thermocline layers as a function of freshwater forcing. . . . . . . . . . . . . . . . 33 
2-2 Surface forcing and geometry for the primitive equation model. All forcing functions vary only in latitude and not in longitude. (a) The prescribed surface air temperature used to compute air-sea heat fluxes. (b) The reference freshwater flux (E-P, evaporation - precipitation) for $E_{0}=1$ in equation (2.8). The magnitude of the freshwater flux is varied across simulations by changing the value of $E_{0}$. (c) A wind stress over the southern re-entrant channel is computed from the zonal wind. (d) The model geometry. We run experiments with two widths $\left(L_{x}\right)$, a narrow Atlantic-like basin, and a wide Pacific-like basin. We included a Scotia-Arc like topographic feature in the channel. . . . . . . . . . . . . . . . . . . . . . . . . . . 39

2-3 The wider basin shows a stronger salinity gradient in the MITgcm simulations. Panel (a) shows the difference in salinity between the northern boundary and 27 degrees north depth-averaged salinity for the narrow and wide basin simulations. The lines stop for the $E_{0 \text { crit }}$ at which convection shuts off. Panels (b) and (c) show the the surface salinity in the narrow (b) and wide (c) basins for a simulation run with $E_{0}=0.5$. . . . . . . . . . . . . . . . 41

2-4 MITgcm simulations show that convection shuts off for a lower freshwater forcing $E_{0 \text { crit }}$ in the wider basin. Panel (a) shows overturning versus freshwater forcing for the narrow and wide basin. Panels (b,c) show overturning in the narrow basin before (b) and after convection shut off (c). . . . . . . . 42

$2-5$ With a wider basin, the overturning is weaker per unit width, but the freshwater fluxes are the same per unit width, leading to a larger salinity gradient and a fresher northern part of the basin. This leads to a less dense convection region, and convection shut-off with weaker freshwater forcing. . . . . . . . . 43

3-1 A schematic describing eddy saturation in the Antarctic Circumpolar Current. When a current is saturated, a change in wind leads to no change in the isopycnal slopes or thermal wind transport. . . . . . . . . . . . . . . . . 46 
3-2 A three-dimensional figure showing the model domain. The color on the surface is a snapshot of the upper-layer potential vorticity for the case with a wind stress maximum $\tau_{0}=0.2 \mathrm{~N} \mathrm{~m}^{-2}$ and residual overturning $\mathcal{H}_{0}=-2$ Sv. The blue surface represents the instantaneous interface height. The wind forcing is shown above the surface. . . . . . . . . . . . . . . . . 50

3-3 A figure showing the streamfunctions for the cases where residual overturning $\mathcal{H}_{0}=-2 \mathrm{~Sv}$ for $(\mathrm{a}, \mathrm{b})$ weak winds of $\tau_{0}=0.0125 \mathrm{~N} \mathrm{~m}^{-2}$ and $(\mathrm{c}, \mathrm{d})$ relatively strong winds of $\tau_{0}=0.2 \mathrm{~N} \mathrm{~m}^{-2}$. Panels (a,c) show the time averaged upperlayer transport, $\overline{\psi_{1}}$, panels $(\mathrm{b}, \mathrm{d})$ show the time averaged lower-layer transport, $\overline{\psi_{2}}$. The black lines indicate where the topography exceeds $100 \mathrm{~m}$.

3-4 Thermal wind transport versus wind stress for four different overturnings with (solid) and without (dashed) a $1000 \mathrm{~m}$ tall ridge. At strong wind stresses there is saturation, but as the wind forcing weakens to moderate, present day, levels, the slopes change with the wind, in different directions depending on the sign of the overturning in order to approach the transport for a flat bottom. When the wind stress is very small and the overturning positive, the flow is westward and will not be discussed further. . . . . . . . . . . . . . . . 58

3-5 Panel (a) shows eddy heat fluxes normalized by the total heat fluxes along the channel for $\mathcal{H}_{0}=-2$ Sv. Most of the heat fluxes occur in or just downstream of the meander. Panel (b) shows the cumulative normalized eddy heat flux along $\mathrm{x}$, indicating that when the wind is strong, most of the heat fluxes occur in the meander, but with weaker winds, the heat fluxes throughout the whole domain are just as important. The black dashed line shows the heat fluxes for a flat-bottomed experiment. . . . . . . . . . . . . . . . . . . . . 59

3-6 Form drag along the channel for $\mathcal{H}_{0}=-2$ Sv. As the wind forcing decreases,

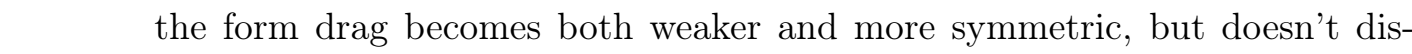
appear altogether, leading to smaller net heat fluxes in the meander (Fig.

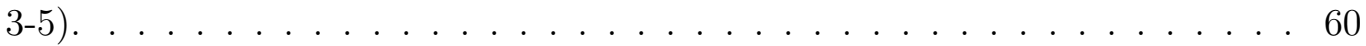


3-7 $\quad$ Thermal wind transport versus residual overturning forced along interface for entire $\mathcal{H}_{0}<0$ range. The linear scaling developed is plotted against the simulated results that works well until the flow becomes eddying. The dashed line shows where the flow reaches its critical shear for instability following the Phillips problem. The thermal wind transport scales directly with the residual overturning and provides the no-wind limit to the thermal wind transport. . 61

3-8 A figure showing the thermal wind transport versus the wind stress and total eddy overturning. The black lines correspond to a constant residual overturning. We see that for a given wind forcing, a stronger eddy overturning corresponds to a stronger thermal wind transport and for a given eddy overturning, a stronger wind forcing corresponds to a weaker thermal wind transport. However, as we increase the wind forcing along a line of constant overturning, the decreasing effect of the wind forcing overpowers the increasing effect of the eddy overturning, leading to smaller thermal wind transports as we increase the wind forcing for a negative residual overturning. . . . . . . . 62

3-9 A concluding schematic. When there is a weak wind forcing, the strength of the thermal wind transport is dependent on the sign of the overturning, the meander is symmetric about the ridge, and the eddy activity is spread throughout the domain. When the wind forcing is strong the eddy activity is localized to the meander region, the meander is asymmetric, and the thermal wind transport no longer depends on the overturning. . . . . . . . . . . . . . 65

4-1 Although the Southern Ocean is often thought about in a zonally symmetric manner, the Southern Ocean indeed has locations where properties, such as surface velocity as shown here, deviate highly from the zonal average. Figure from Chapman et al. || $2015 \mid . \ldots$. . . . . . . . . . . . . . . . . 69

4-2 Geometry of the idealized channel. Ridge at $800 \mathrm{~km}$, wind has a sine profile with a maximum $\tau_{0}$, the color here is snapshot temperature with $\tau_{0}=0.15 \mathrm{~N}$ $\mathrm{m}^{-2}$ 
4-3 Figure showing the temperature boundary conditions. (a) Northern boundary relaxation temperature. The temperature is relaxed linearly from the maximum at the northern boundary, linearly scaling back to no forcing at 1930 $\mathrm{km}$. (b) Heat flux profile used with the average surface temperature (c) to generate surface relaxation temperature. (d) shows the full heat flux pattern

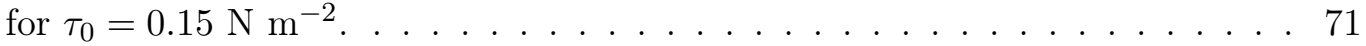

4-4 Residual overturning in temperature space (a,c). The same overturning projected into depth coordinates $(\mathrm{b}, \mathrm{d})$. The overturning is shown for flat-bottomed simulations $(\mathrm{a}, \mathrm{b})$ and ridged simulation $(\mathrm{c}, \mathrm{d}) . \ldots \ldots \ldots$. . . . . . . . 73

4-5 Overturning strength versus wind stress maximum for the ridge simulation. The upper cell is the maximum overturning, the lower cell is the minimum at the northern boundary and meander cell is the minimum throughout the domain. . . . . . . . . . . . . . . . . . . 74

4-6 The vertical displacement of particles as they exit their initial boxes. We split the vertical displacement into mean and eddy and then further to the barotropic and baroclinic eddies. The blue line separates the upper and lower cells, the green line separates the upwelling and downwelling in the lower cell. The time required for particles to leave each box varies widely depending on the horizontal velocities with order 10 days on average. . . . . . . . . . . 75

4-7 $\quad$ Vertical displacement along particle trajectories from the alternate particle tracking method showing how the the displacement behaves over a long time

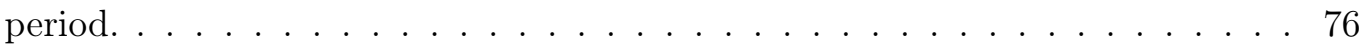

4-8 A figure showing the mean vertical velocities calculated from particles and from MITgcm output over a 5 day period. They agree to lowest order, however they are not identical as $\mathrm{w}$ calculated on an isopycnal not identical to the Eulerian $\mathrm{w}$ calculated in the model. . . . . . . . . . . . . . . . . . . . . 77

4-9 Particle tracking of particles released at the channel entrance in both 5 day averaged and 1 day averaged velocities. The difference shows more upwelling for the 1 day average, indicating that it is necessary to appropriately represent upwelling. . . . . . . . . . . . . . . . . . . . . . . . 77 
4-10 A figure showing the difference between 1 year of particle releases and 2 years of particle releases with particles released every 30 days, testing the length of the averaging period. The difference here is primarily noise, indicating that one year is sufficient for our purposes. . . . . . . . . . . . . . . . . . 78

4-11 Displacement versus X split among different components for different temperature bins that represent the different overturning cells. This displacement is the amount particles upwell in the box, a kind of weighted velocity that weights how long a particle spends in each cell. We generally see upwelling before the ridge at $800 \mathrm{~km}$ and downwelling after. . . . . . . . . . . . 87

4-12 Zonally-averaged displacement of particles in a flat bottomed channel where $\tau_{0}=0.15 \mathrm{~N} \mathrm{~m}^{-2}$ in $\mathrm{m}$. (a) shows the total vertical displacement (b) shows the mean, and (c) shows the eddies. . . . . . . . . . . . . . . . 88

4-13 Just like figure 4 -11 $\mid$ except for $\tau_{0}=0.2 \mathrm{~N} \mathrm{~m}^{-2}$. Notice that baroclinic eddies are significantly weaker downstream for the stronger wind. . . . . . . . . . . 89

4-14 A figure schematically comparing our old understanding of the overturning to our new understanding. Instead of just wind and eddies, there is now a topographic component and the eddies are localized. The topographic component, also called standing eddy component, is working in concert with the wind in the downstream region of topography. Note here that in our simulations the upper cell downwelling occurs entirely at the northern boundary, so we don’t represent these processes in the schematic. . . . . . . . . . . . . . . 90

5-1 A figure showing a DIC section for $\tau_{0}=0.15$. Note the difference between \begin{tabular}{|c|}
\hline the northern boundary and the interior. Black contours show temperature \\
\hline
\end{tabular} contoured every degree. At the northern boundary we relax to GLODAPv2 preindustrial DIC . . . . . . . . . . . . . . . . . . 94

5-2 Air-sea carbon fluxes with contours showing the barotropic streamlines. The boxes indicate the regions where we compute the difference in $\mathrm{pCO}_{2}$, used in a previous analysis. . . . . . . . . . . . . . . . . . . . . . 95

5-3 Figure showing the air-sea carbon fluxes averaged over the whole domain versus the wind stress maximum. . . . . . . . . . . . . . . 96 
5-4 Figure showing the contribution of different terms to air-sea carbon flux differences (weak wind $\left(\tau_{0}=0.05 \mathrm{~N} \mathrm{~m}^{-2}\right)$ - moderate wind $\left.\left(\tau_{0}=0.15 \mathrm{~N} \mathrm{~m}^{-2}\right)\right)$ in $\mu$ atm. (a) shows the total difference in $\mathrm{pCO}_{2}$ fluxes, (b) shows the contribution from DIC, (c) shows the contribution from Alk, and (d) shows the contribution from temperature. . . . . . . . . . . . . . . . . . 97

B-1 Schematic relating eddy orientation and stability (both barotropic and baroclinic). The black arrows to the left show the shear related to the mean flow $u$, the dashed lines are the eddy stream function contours $\psi^{\prime}$. Purely barotropic eddies tilt in the horizontal (top) and $\psi^{\prime}$ is a function of $u^{\prime}$ and $v^{\prime}$; purely baroclinic eddies tilt in the vertical (middle) and $\psi^{\prime}$ is a function of $v^{\prime}$ and $b^{\prime}$. When the eddy major axis tilts with the shear, the eddies are stable and decay, giving energy to the mean flow, which increases the shear. When the eddy major axis tilts against the shear, the eddies are unstable and grow, taking energy from the mean flow, which reduces the shear. . . . . . . . . . . 126

B-2 Eddy kinetic energy at $177 \mathrm{~m}$ depth for experiments with wind stress forcing (a) $\tau_{0}=0.1 \mathrm{~N} \mathrm{~m}^{-2}$ and (b) $\tau=0.2 \mathrm{~N} \mathrm{~m}^{-2}$. Thick white contours indicate the region containing EKE that is at least $80 \%$ of the peak EKE. Thin lines show potential temperature contours and are separated by $1^{\circ} \mathrm{C}$. Thick lines show where the Gaussian ridge, centered at $800 \mathrm{~km}$, rises above $3800 \mathrm{~m}$ depth. 127

B-3 Depth-integrated energy conversion terms $\left(\mathrm{W} \mathrm{m}^{-2}\right)$ for the experiment with $\tau_{0}=0.1 \mathrm{~N} \mathrm{~m}^{-2}$. Contour lines show temperature every degree Celsius at 177 m depth. $D_{P_{M}}$ is the rate of change of MAPE due to horizontal eddy fluxes (B.5), $D_{P_{E}}$ is the rate of change of eddy energy due to horizontal eddy density fluxes (B.6), $D_{K_{E}}$ is the rate of change in EKE from EAPE (B.7), $M_{K_{M}}$ is the rate of change in MKE due to eddy momentum fluxes (B.8) and $M_{K_{E}}$ is the rate of change in EKE due to eddy momentum fluxes (B.9). . . . . . . . 128

B-4 Depth-integrated energy conversion terms $\left(\mathrm{W} \mathrm{m}^{-2}\right)$ with $\tau_{0}=0.2 \mathrm{~N} \mathrm{~m}^{-2}$.

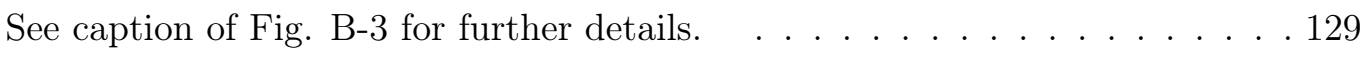


B-5 Lorenz diagram from two different regions, indicated by the boxes in Figs. B3 and $\mid \mathrm{B}-4$. The meander region is located just downstream of the ridge and the downstream domain refers to a region with quasi-zonal jets. The values provided in the diagram are depth-integrated, domain-averaged quantities; the units are $\mathrm{W} \mathrm{m}^{-2}$. The diagrams follow those shown in Chen et al. $\mid$ [2014|. 130

B-6 $\quad$ Depth-averaged eddy anisotropy in color (a) and the tilt (b) for $\tau_{0}=0.1 \mathrm{~N}$ $\mathrm{m}^{-2}$; both diagnostics are dimensionless. The color shows the magnitude of the velocity at $177 \mathrm{~m}$ depth. The temperature is contoured every degree at $177 \mathrm{~m}$ depth and the bold line is a streamline computed from the velocities and is used in Fig. $|\mathrm{B}-8| \ldots \ldots \ldots 131$

B-7 Depth-averaged eddy anisotropy in color (a) and the tilt (b) for $\tau_{0}=0.2 \mathrm{~N}$ $\mathrm{m}^{-2}$; both diagnostics are dimensionless. The color shows the magnitude of the velocity at $177 \mathrm{~m}$ depth. The temperature is contoured every degree at $177 \mathrm{~m}$ depth and the bold line is a streamline computed from the velocities and is used in Fig. $|\mathrm{B}-9|\rceil \ldots$. . . . . . . . . . . . . . . . . . 132

B-8 (a) Vertical eddy anisotropy and (b) the tilt into the vertical with respect to the mean flow (Eq. 16) for $\tau_{0}=0.1 \mathrm{~N} \mathrm{~m}^{-2}$. The calculations are complete along the streamline shown in Fig. $\mid \mathrm{B}-6.6$. The solid black lines show the location of the •'s in (c) and the dashed line shows the location of the $*$ in (c). A positive tilt (red) represents down-gradient buoyancy fluxes, and a negative (blue) tilt represents up-gradient buoyancy fluxes. (c) A measure of the horizontal shear $|\nabla u|^{2}+|\nabla v|^{2}$ with the streamline followed in (a) and (b). 133

B-9 (a) Vertical eddy anisotropy and (b) the tilt into the vertical with respect to the mean flow (Eq. 16) for $\tau_{0}=0.2 \mathrm{~N} \mathrm{~m}^{-2}$ along the streamline shown in Fig. $\mid$ B-7| Details follow Fig. $\mid$ B-8, . . . . . . . . . . . . . . . . . . . . . 133 
B-10 Schematic summarizing the various regimes in the ACC-like channel; critically there are multiple regimes within the meander region itself. Colors indicate the direction of the eddy buoyancy and momentum fluxes. Upstream of the meander as the flow encounters the topography, the convergence of streamlines both in the horizontal and vertical leads to an increase in eddy anisotropy. Immediately downstream of the ridge crest, the strong rotation of flow direction leads to up-gradient buoyancy fluxes and up- and down-gradient momentum fluxes. The flow becomes barotropically and baroclinically unstable resulting in rapid generation of eddy kinetic energy (EKE). Downstream, zonal jets develop and baroclinic instability dominates with up-gradient momentum fluxes. . . . . . . . . . . . . . . . . . . . . . . . . . 134 


\section{List of Tables}

3.1 Table showing various parameters used for two-layer quasi-geostrophic model. 49

$5.1 \quad$ Biological parameters used in the MITgcm simulation and their values. . . . . 93 


\section{Chapter 1}

\section{Introduction}

The ocean circulates water in all three dimensions; however, it prefers to move nearly horizontally, along surfaces of constant density, changing longitude and latitude but not across density surfaces. Because of this, the vertical transport of water and the underlying vertical velocities tend to be smaller than the horizontal ones. Despite this, the vertical transport of water is vital for understanding the connection between the ocean and climate. The vertical transport of water occurs in specific locations creating a global connected circulation loop. This circulation is traditionally called the conveyer belt circulation Broecker et al., 1991], although that calls to mind a somewhat misleading picture, since the transport is turbulent and occurs over a broad area. Water densifies and sinks to depth in two main places (Figure 1-1). The first location is the North Atlantic where heat loss leads to densification and water sinking to the bottom in a process called deep convection. Interestingly, there is not an equivalent sinking zone in the North Pacific. Chapter 2 of this thesis addresses an underlying physical mechanism behind why there is deep convection in the North Atlantic but not the North Pacific.

The other main location for water densification and sinking is the Southern Ocean. Studies of the circulation of the Southern Ocean comprise the remaining chapters of this thesis. In the Southern Ocean (the ocean around the continent of Antarctica), primarily in the Weddell and Ross Seas, brine rejection from sea ice formation densifies water where it sinks to the bottom of the global ocean, creating the densest water in the global ocean Marshall and Speer, 2012. The Southern Ocean is also key for the upward transport of old, deep water. The deep waters have been below the surface, after being created in the 
North Atlantic, or the Southern Ocean, for hundreds and thousands of years, respectively, where over time little organisms die, sink, and decompose, increasing carbon content in the old, deep water. When these waters are exposed to the atmosphere in the Southern Ocean they both release the carbon dioxide gas that had been building up for centuries and take up new human-sourced carbon dioxide. Thus, the vertical transport of water is relevant for understanding air-sea carbon fluxes. Similarly, these areas are important for heat uptake by the Southern Ocean. Because of its relevance, the Southern Ocean circulation that leads to upwelling and downwelling is the topic of the remaining 3 chapters.

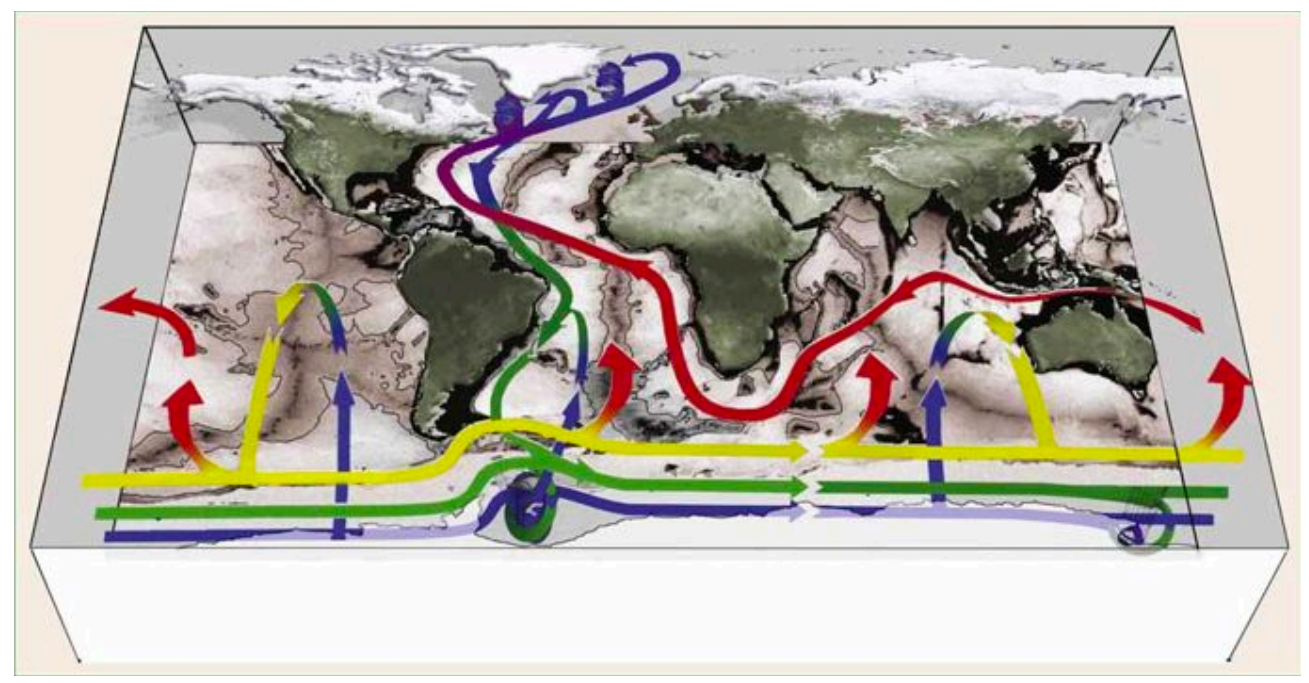

Figure 1-1: This figure shows the conveyor belt circulation from Marshall and Speer 2012. The colors represent different temperatures of water. The primary locations of sinking water are the North Atlantic and the Weddell and Ross Seas. The primary location of rising water is in the Southern Ocean. These are not actual trajectories of water because variability is significant in the actual movement of water parcels.

Over the course of this thesis we approach the problem of vertical ocean transport using a combination of theory and idealized ocean modeling. We can conceptualize the large-scale circulation as a two-dimensional circulation (in depth versus latitude) called the meridional overturning circulation. In order to reduce the three-dimensional circulation to two dimensions, we take averages in longitude, called zonal averages. Often, the community studies the meridional overturning circulation using numerical models, both realistic and idealized. We add to this approach by also using conceptual models, highly-idealized approximations of the system containing only the necessary physics. The combination of these techniques provides us powerful leverage to understand the role of basin width in setting the overturning. 
Specifically, we look at the role of basin width for it is the lowest order difference between the North Atlantic and the North Pacific. In the Southern Ocean, we are interested in the role that a zonal asymmetry in topography plays in modifying the overturning circulation, similar in spirit to the basin width experiments used to understand the overturning.

In this thesis we developed theory for meridional overturning circulation using several techniques, including a hierarchy of models of increasing complexity to understand how individual factors contribute to the full system behavior Maher et al. 2019. In a hierarchy, the most basic models are governed by simple systems of equations with a limited number of layers in the vertical and idealized geometry and forcings Youngs et al., 2019]. A next step in the hierarchy might be a model with the same simplified geometry and forcings but with a continuous framework in the vertical Youngs et al., 2017. Next, a carbon model was added to this model (Chapter 5). In this way, additional levels of complexity are added one by one, carrying an understanding of the key dynamical processes through each level of the hierarchy until we can show by comparison to observations which specific processes are driving the real world behavior. We use this framework of a hierarchy of models to answer several different questions about the zonal asymmetry of the overturning circulation.

\subsection{Northern Deep Convection}

The deep convection of the North Atlantic and the lack thereof in the North Pacific has puzzled many over the last 50 years [e.g. Warren, 1983; Nilsson et al., 2013, Weijer et al., 1999, Jones and Cessi, 2016; Ferreira et al., 2018]. The basic controlling factors for deep convection and its response to salinity changes are highly relevant for past and future climate, in part because the climate system and its relevant forcings for the meridional overturning could have been or will be very different. Many different proposed theories suggest that the difference in circulation between the North Atlantic and the North Pacific is due to asymmetries in air-sea fluxes, fluxes of salt between basins, or gyre circulation Warren, 1983 , Ferreira et al., 2010, Nilsson et al., 2013, Weijer et al., 1999, Jones and Cessi, 2017, 2016.

Previous studies have addressed this problem by testing the effectiveness of different mechanisms using modeling. 


\subsection{Southern Ocean Overturning}

The Southern Ocean is responsible for about 30\% to $40 \%$ of oceanic anthropogenic carbon uptake, an oversized contribution Gruber et al., 2009; Frölicher et al., 2015. The main process for vertical transport of tracers in the Southern Ocean is the residual overturning circulation, so the overturning is likely a leading process in regulating air-sea carbon fluxes in the Southern Ocean Marshall and Speer, 2012]. Currently there is little understanding of how the residual overturning in the Southern Ocean responds dynamically to changes in wind stress and buoyancy forcings, with most studies considering the steady state overturning and constant forcings Hallberg and Gnanadesikan, 2006, Abernathey et al., 2011; Meredith et al., 2012. Since the overturning is dominated by local mesoscale instabilities generated by topography [Youngs and Flierl, in prep.] (Chapter 4), how these processes respond to climate change over a variety of time scales is relevant for global carbon budgets. I have investigated energy budgets of the system as forced by varying winds, which gives insight into the types of turbulence arising from the Southern Ocean Youngs et al. 2017] (Appendix B). One type of turbulence, called barotropic instability, less important with a flat bottom, but becomes leading order with topography, indicating that the topography is fundamental for the dynamics. The barotropic instability is not parameterized in leading global climate models, so its dominance in my simulations points to a shortfall of those models.

\subsection{Summaries}

\subsubsection{Basin-Width Dependence of Northern Deep Convection}

This study provides a simple explanation for why there is a preference for deep convection in the North Atlantic but not the North Pacific. We combine fundamental scaling laws for the overturning with a salt budget in a simple box model to show that a wider basin has a stronger north-south salinity gradient due to relatively-weaker overturning and that convection shuts off at a weaker freshwater forcing. Single-basin MITgcm simulations corroborate the lowest-order behavior of the box model. Our results highlight the utility of simple box models for understanding complex nonlinear behavior. 


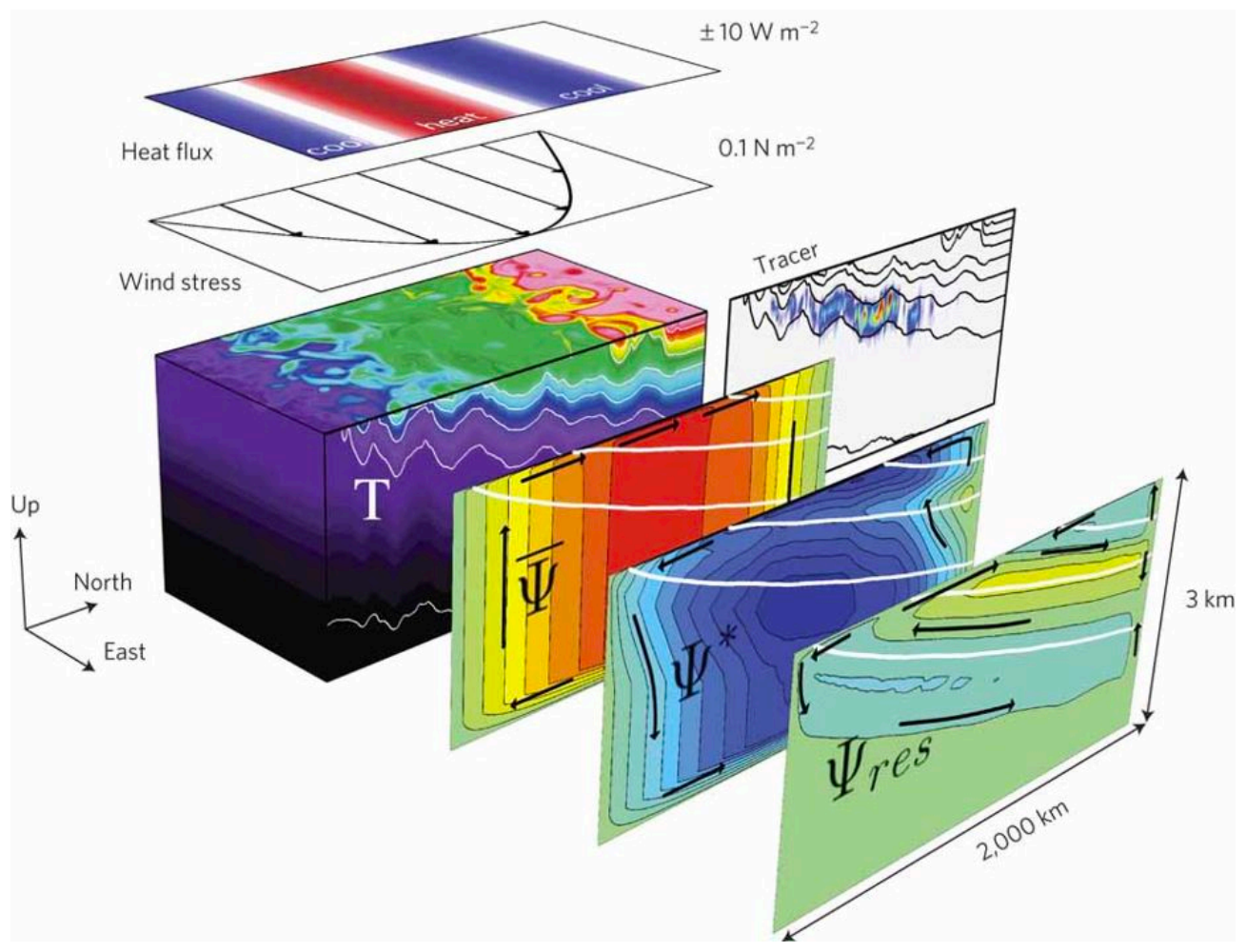

Figure 1-2: This figure schematically shows our understanding of the overturning circulation. The wind blows over the Southern Ocean-like channel inducing a clockwise mean circulation, tilting up isopycnals. The eddy driven counter-clockwise circulation acts to flatten isopycnals, leading to the residual circulation resulting from the partial cancellation of the components. Figure from Marshall and Speer 2012.

\subsubsection{Role of Residual Overturning for the Sensitivity of Southern Ocean Isopycnal Slopes to Changes in Wind Forcing}

For the next chapter, we pivot from the global overturning circulation to Southern Ocean dynamics, as the Southern Ocean plays a significant role in the global circulation. We tested the response of the stratification and transport in the Southern Ocean to changes in wind using a highly-idealized two-layer quasi-geostrophic model Youngs et al. 2019 (Chapter 3). In addition to showing that meanders are necessary to reproduce the behavior of the Southern Ocean, we found that strong winds concentrate the baroclinic and barotropic instabilities downstream of the bottom topography and weaken the instabilities elsewhere due to a form-drag process. With weak winds, however, the system is essentially symmetric in longitude, like a flat-bottomed ocean. This result is consistent with observations of elevated turbulence downstream of major topography in the Southern Ocean. One advancement 
made by this study is that we introduced a way to simply model density changes and the resulting overturning circulation, which was previously utilized in the atmospheric literature but had been rarely applied to ocean modeling [e.g Schneider, 2006].

\subsubsection{Causes of Localized Upwelling in a Southern Ocean-like Channel Near Topography}

For the next thesis chapter, we apply the two-layered results to a more realistic Southern Ocean-like channel, with and without bottom topography, and showed how the large-scale circulation varies with wind (Chapter 4). In addition, we examined the three-dimensional circulation in order to understand where vertical transport occurs and developed a picture of the pathways taken by each individual water parcel (Chapter 4). We found that the vertical transport happens in very isolated locations, just downstream of topography and in addition the time-mean flow leads to specific upwelling patterns. This suggests that further investigations close to topography are necessary to understand the overturning.

\subsubsection{Localized Air-sea Carbon Fluxes due to Topography in a Southern Ocean-like Channel}

As a final step we add another layer of complexity in order to connect this theoretical work to real-world applications (Chapter 5). We added a biogeochemical model to my MITgcm channel simulations in order to investigate the role of zonal asymmetries on the air-sea carbon fluxes. We find that carbon fluxes are enhanced near topography, again highlighting the role of zonal asymmetries. The modeling technique that we use is a simple biogeochemical model for the MITgcm Lauderdale et al. 2016]. We approach the biogeochemical modeling from a highly idealized perspective, which is a bit unique among biogeochemical studies which tend to use more realistic models and geometry. This chapter reports on our initial studies.

\subsubsection{Conclusion}

Finally, I conclude my thesis by providing an additional perspective on how my thesis has added to the Physical Oceanography literature. In addition, I discuss my views on the state of the field including what are the most relevant scientific questions, what challenges we as a field face to answering those questions, and what research tools can be used to address 
these challenges. This chapter succeeds in putting this work into the broader context of the field. 


\section{Chapter 2}

\section{Basin-Width Dependence of Northern Deep Convection}

\section{$2.1 \quad$ Introduction}

The paleo record suggests that deep convection in the North Atlantic turned on and off many times over the last few million years resulting in major climate shifts Boyle 1990; Thornalley et al. 2011. For example, the shut down of deep convection is believed to have triggered the cold Heinrich events, while the turning on of deep convection would have promoted the rapidly warming Dansgaard-Oeschger events Walin 1985;; Timmermann et al. 2003]; Loving and Vallis 2005. There is also speculation that convection may shut off again in a future warmer climate Rahmstorf et al., 2015, Sgubin et al., 2017. Accordingly, understanding what controls high latitude convection is key to a complete theory of past, present, and future climate.

Explanations for why convection occurs in the North Atlantic but not in the North Pacific have focused on the different roles of heat and salt on the overturning Ferreira et al. [2018]. Ferreira et al. [2010] pointed to the larger freshwater flux received by the North Pacific Ocean because of its larger width compared to the North Atlantic Ocean. Jones and Cessi 2017] highlighted the importance of Northern Hemisphere wind-driven gyres (and their width dependence) on the salt budget and overturning state of each basin. A few studies Nilsson et al., 2013, Weijer et al., 1999, Jones and Cessi, 2016 contended that the inter-basin transport of salt between the Atlantic and Indo-Pacific basins accounts for 
the difference in overturning states. Here we wish to demonstrate that in addition, and independently of these mechanisms, the different longitudinal extent of the North Atlantic and North Pacific basins also favors convection in the narrow basin through its impact on the overturning. Our goal is not to identify which among the proposed mechanisms is most important in any specific climate. Rather we derive a scaling law to estimate the impact of freshwater fluxes on high latitude convection building on recent advances in the theory of the global overturning circulation and stratification Gnanadesikan, 1999 Shakespeare and Hogg, 2012; de Boer and Hogg, 2014; Nikurashin and Vallis, 2011, 2012, Gnanadesikan et al. [2018]. Our hope is that this scaling can then be used together with those proposed for inter-basin and gyre freshwater transports to quantify the relative importance of each process in more realistic simulations of the global ocean circulation.

We approach this problem by extending the two-layer model of Gnanadesikan [1999 to account for the different boundary conditions for temperature and salinity (restoring for the former and flux for the latter), which are believed to be crucial in selecting whether deep convection develops in the North Atlantic Stommel, 1961; Marotzke et al., 1988; Johnson et al. 2007. We then examine the dependence of overturning and convection shut-off on the width of the basin in both the two-layer analytical model in section 2 and in a full primitive equation model with idealized geometry in section 3 . We discuss and conclude in section 4 .

\subsection{Two-layer Analytical Model}

We set up a conceptual model of the ocean overturning circulation to understand salt's role in deep convection by extending the approach of Gnanadesikan 1999. We consider a model consisting of two layers, a thermocline layer (layer t) of depth $h$ and a deep layer (layer d), a setup similar to that used by Johnson et al. 2007] and sketched in Figure 2-1. Each layer has a uniform temperature and salinity given by $T_{t}, S_{t}$ and $T_{d}, S_{d}$, respectively. There is a positive area-integrated freshwater flux $\mathcal{P}$ in $\mathrm{m}^{3} \mathrm{~s}^{-1}$ into the deep layer, acting to freshen it, and a surface freshwater flux of $-\mathcal{P}$ out of the thermocline layer, acting to make it saltier. The temperatures $T_{t}, T_{d}$ are fixed to constant values and $T_{t}>T_{d}$. These choices are made to capture the asymmetry in surface forcing of salt and heat: a freshwater flux for salinity and a restoring to the prescribed atmospheric value for temperature [Haney, 1971].

The transport of waters from the thermocline to the deep layer by deep convection is 


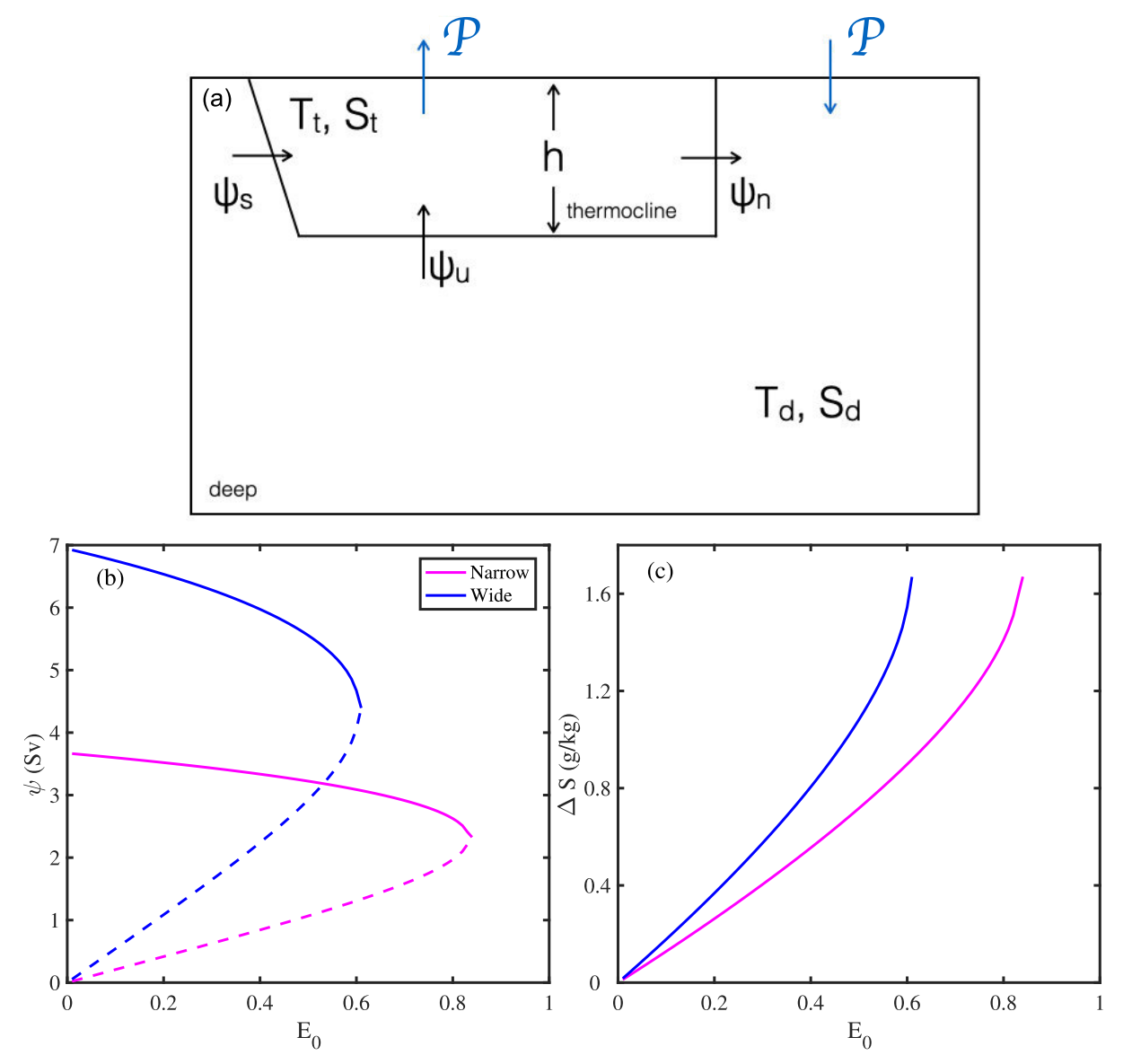

Figure 2-1: (a) Schematic of the conceptual model configuration. The ocean is divided in two layers of uniform temperature and salinity: a thermocline layer and a deep layer. A freshwater flux of $\mathcal{P} \mathrm{m}^{3} \mathrm{~s}^{-1}$ reduces salinity in the deep layer and an equal and opposite freshwater flux increases salinity in the thermocline layer, representing the dominance of precipitation at high latitudes and of evaporation at low latitudes. There are transports between the two layers: $\psi_{n}$ represents convection which converts light to dense water, $\psi_{u}$ represents the reverse conversion through deep ocean mixing and $\psi_{s}$ through meridional surface flows in a "Southern Ocean". (b) $\psi_{n}$ as a function of freshwater forcing, with the solid lines as the stable solutions, and dashed lines as the unstable solutions. (c) The difference in salinity between the deep and the thermocline layers as a function of freshwater forcing.

represented by $\psi_{n}$, the diffusive diabatic upwelling from the deep layer to the thermocline is represented by $\psi_{u}$, and the upwelling from the deep layer to the thermocline layer though a southern re-entrant channel is given by $\psi_{s}$, following a scaling meant to capture the dynamics of the Southern Ocean. At equilibrium the mass budget for the model requires that $\psi_{n}=\psi_{s}+\psi_{u}$. 


\subsubsection{Scalings for Overturning}

The sinking of waters associated with deep convection in the northern hemisphere is assumed to follow the scaling first proposed by Gnanadesikan [1999],

$$
\psi_{n}=\frac{\Delta b h^{2}}{f}
$$

where $\Delta b$ is the difference in buoyancy between the thermocline and the deep layer, $h$ is the depth of the thermocline layer, and $f$ is the high latitude Coriolis parameter. In this analytical model, $\Delta b=g\left(\alpha\left(T_{t}-T_{d}\right)-\beta\left(S_{t}-S_{d}\right)\right)=g(\alpha \Delta T-\beta \Delta S)$ where $\alpha$ is the thermal expansion coefficient and $\beta$ is the saline contraction coefficient, $\Delta T=T_{t}-T_{d}$ and $\Delta S=S_{t}-S_{d}$ (both positive values).

The diabatic, diffusive upwelling is expressed as,

$$
\psi_{u}=\frac{\kappa_{v}}{h} A
$$

where $\kappa_{v}$ is the vertical diffusivity, and $A$ is the total area of the basin where upwelling occurs Munk, 1966, Nikurashin and Vallis, 2012. This scaling represents a vertical advectivediffusive balance for buoyancy in the ocean interior. We keep the area of diffusive upwelling general in order to connect our scalings to the present day ocean where upwelling happens primarily in the Indo-Pacific and not in the convecting basin Ferrari et al., 2017, Newsom and Thompson, 2018; Forget and Ferreira, 2019; Holmes et al., 2019.

The adiabatic upwelling in a southern channel is given by the residual between Ekman transport acting to steepen isopycnals and eddies acting to relax them Marshall, 1997; Gnanadesikan, 1999, Marshall and Radko, 2003,

$$
\psi_{s}=\left(\frac{\tau}{\rho_{0} f}-K_{e} \frac{h}{\ell}\right) L_{x}
$$

where $\tau$ is the wind stress blowing over the channel, $\rho_{0}$ is a reference density, $K_{e}$ is the Gent-McWilliams eddy diffusivity, and $h / \ell$ is the slope of the density surface outcropping in the southern channel ( $\ell$ is the meridional extent of the slumping isopycnal in Fig. 2-1).

Two limits are potentially relevant for the ocean circulation: a diabatic limit where the northern convection deep waters are transformed back into thermocline waters through diabatic upwelling in the basin i.e. $\psi_{n} \simeq \psi_{u}$, and an adiabatic limit where the northern 
convection deep waters upwell adiabatically to the surface in the southern channel where they are transformed back into thermocline waters through surface heat fluxes i.e. $\psi_{n} \simeq \psi_{s}$. We will focus our analysis to the diabatic limit which is mathematically simpler. However, as we point out below, the adiabatic limit results in a qualitatively similar dependence of the overturning circulation on basin width, which is the key ingredient to explain why convection is favored in a narrower basin like the Atlantic. It is reassuring that the overturning displays the same qualitative dependence on basin width in both limits, because the numerical simulations described below and likely the real ocean are in a regime in between the two limits.

In the diabatic limit, $\psi_{n}=\psi_{u}=\psi$, and we can equate Eq. 2.1) and Eq. 2.2.,

$$
\frac{\Delta b h^{2}}{f}=\frac{\kappa_{v}}{h} A
$$

and solve for $h$,

$$
h=\left(\frac{f \kappa_{v} A}{\Delta b}\right)^{1 / 3} .
$$

Substituting $h$ back into Eq. 2.1 or 2.2 gives a scaling for the overturning,

$$
\psi=\frac{\Delta b^{1 / 3}\left(\kappa_{v} A\right)^{2 / 3}}{f^{1 / 3}}
$$

This relationship cannot be solved for $\psi$, because the buoyancy difference $\Delta b$ depends on the salinity difference which in turns depends on the strength of the overturning circulation as we show next.

\subsubsection{Salt Budget}

The temperature difference $\Delta T$ between the two layers is fixed, because ocean temperatures are quickly restored to the overlying atmospheric value Haney [1971]. The salinity difference is instead the result of a balance between the air-sea freshwater flux and the salt exchange between the two layers,

$$
\begin{aligned}
\frac{\partial S_{t} V_{t}}{\partial t} & =\mathcal{P} S_{0}+\psi\left(S_{d}-S_{t}\right) \\
\frac{\partial S_{d} V_{d}}{\partial t} & =-\mathcal{P} S_{0}+\psi\left(S_{t}-S_{d}\right)
\end{aligned}
$$


where $S_{0}$ is a reference salinity, $\mathcal{P}$ is a positive area-integrated freshwater flux in $\mathrm{m}^{3} \mathrm{~s}^{-1}$. $S_{0} \mathcal{P}$ is the virtual salt flux and it is equal and opposite in the two layers. $V_{t}, V_{d}$ are the volumes of the thermocline and deep layers. Steady state solutions are obtained subtracting the two expressions and remembering that $\Delta S=S_{t}-S_{d}$ to find

$$
\Delta S=\frac{\mathcal{P}}{\psi} S_{0}
$$

The salinity difference $\Delta S$ is positive $\left(\mathcal{P}, S_{0}\right.$ and $\psi$ are all defined positive) because evaporation over the thermocline keeps its salinity higher than that of the deep waters. This tendency is offset by the overturning $\psi$ which acts to reduce $\Delta S$. The freshwater flux $\mathcal{P}$ and the overturning $\psi$ do not scale in the same way with basin size, as we discuss next. Thus the salinity difference $\Delta S$ (and thus the buoyancy difference $\Delta b$ ) between thermocline and deep waters will depend on basin size. Since $\Delta b$ sets the strength of the sinking at high latitudes, this further implies that the strength of convection depends on basin size. This is the fundamental insight of our study.

The freshwater flux per unit area is comparable in the North Atlantic and North Pacific Forget et al. 2015. Given that we focus on the impact of the basin width-the major geometrical difference between the North Pacific and North Atlantic-we keep the basin length fixed and write the net freshwater flux as $\mathcal{P}=E_{0} L_{x} p$, where $p$ is the freshwater flux per unit zonal length in $\mathrm{m}^{2} \mathrm{~s}^{-1}, L_{x}$ is the basin width and $E_{0}$ is a dimensionless variable scaling factor that we use to vary the overall magnitude of the freshwater forcing. $E_{0}=1$ represents present day levels of freshwater fluxes.

\subsubsection{Overturning Shut-off}

Consistent with previous analytical and climate model studies Rahmstorf, 1996, Dijkstra. 2007; Johnson et al. 2007; Gnanadesikan et al., 2018, our model predicts that convection shuts down when the freshwater flux exceeds a critical threshold value. This is demonstrated by substituting the expression for $\Delta S$, equation (2.5), in the expression for $\psi$, equation 2.1. We obtain an expression for $\psi$ that contains only independent parameters.

$$
\psi=\frac{g^{1 / 3}\left(\alpha \Delta T-\beta E_{0} L_{x} p S_{0} / \psi\right)^{1 / 3}\left(\kappa_{v} A\right)^{2 / 3}}{f^{1 / 3}} .
$$


This expression can be converted into a quartic equation and the roots found. A plot $\psi$ as a function of $E_{0}$ for two different values of basin width is shown in Fig $1 \mathrm{~b}$ for $L_{x}=3.7 \times 10^{6}$ $\mathrm{m}$ ( narrow basin) and $L_{x}=9.6 \times 10^{6} \mathrm{~m}$ (wide basin, 2.6 times wider than the narrow basin). The other parameters are set to $g=9.8 \mathrm{~m} \mathrm{~s}^{-2}, \alpha \Delta T=1 \times 10^{-3}, \beta=4.5 \times 10^{-4} \mathrm{~kg}$ $\mathrm{g}^{-1}, \kappa_{v}=1.2 \times 10^{-5} \mathrm{~m}^{2} \mathrm{~s}^{-1}, S_{0}=35 \mathrm{~g} \mathrm{~kg}^{-1}, f=1 \times 10^{-4} \mathrm{~s}^{-1}$, and $p=0.0357$ $\mathrm{m}^{2} \mathrm{~s}^{-1}$. For simplicity we now consider a single basin geometry and thus set $A=L_{x} L_{y}$ with $L_{y}=1.6 \times 10^{7} \mathrm{~m}$. A solution with $\psi>0$, and thus convective sinking, exists only for $E_{0}$ smaller than a critical value $E_{0 \text { crit }}$ as shown in Fig. 2-1 b. Furthermore the critical freshwater forcing $E_{0 c r i t}$ is larger in the narrow basin $\left(E_{0 c r i t}=0.84\right)$ than in the wide basin $\left(E_{0 \text { crit }}=0.61\right)$.

We can calculate an analytical expression for $E_{0 c r i t}$ by noticing that at $E_{0 c r i t}, d E_{0} / d \psi=$ 0. Some algebra gives,

$$
E_{0 \text { crit }}=\frac{3}{4^{4 / 3} p} \frac{(\alpha \Delta T)^{4 / 3} g^{1 / 3}\left(\kappa_{v} A\right)^{2 / 3}}{\beta f^{1 / 3} L_{x} S_{0}}
$$

The key result here is that $E_{0 c r i t}$ scales inversely with basin width. In the one basin model solved numerically $A=L_{x} L_{y}$ and thus $E_{0 c r i t} \propto L_{x}^{-1 / 3}$, while in a more general situation where the upwelling happens in a different basin than the convecting one then $A$ is fixed

and $E_{0 c r i t} \propto L_{x}^{-1}$. In either case convection shuts off for a weaker freshwater forcing in a wider basin.

In our model convection shuts off through a "saddle node bifurcation": for $E_{0}<E_{0 \text { crit }}$, there are two real roots (and a complex pair). For $E_{0}>E_{0 c r i t}$, there are no purely real roots (two complex pairs instead). Time-evolving solutions diverge from the dashed line; above $E_{0 c r i t}$, the move downward. The downward moving solutions generate unstable stratification and the model ceases to be realistic.

\subsubsection{Adiabatic limit}

It is straightforward to repeat the above analysis for the adiabatic limit, i.e. when $\psi_{n}=\psi_{s}$. Nikurashin and Vallis 2012 derive the expression for $\psi$ in this limit. Following the same steps as above, one can compute the bifurcation diagram for $\psi$ as a function of $E_{0}$. Much like in the diabatic limit, the wider basin has a smaller $E_{0 c r i t}$ than the narrow basin, but the dependence on $L_{x}$ is weaker than in the diabatic limit. Since a wider basin has a smaller 
critical freshwater forcing in both the diabatic and adiabatic limits, we expect this result to hold in between the two limits as well.

\subsection{Primitive Equations Model}

\subsubsection{Configuration}

To lend credence to the analytical model, we run simulations with a full primitive equation model. We do not expect the primitive equation solutions to behave exactly like the analytical model, but do anticipate the same qualitative behavior. We use the MITgcm in a $2^{\circ}$ horizontal resolution configuration with a northern basin and a re-entrant channel in the south shown in Fig. 2-2 [Marshall et al., 1997]. We use 40 vertical layers ranging from $37 \mathrm{~m}$ thick at the surface to $159 \mathrm{~m}$ thick at the bottom. We perform single-basin experiments to test the impact of basin width on the overturning, with spherical sectors of width $L_{x}=56^{\circ}$ for the narrow basin, giving a basin area close to that of the Atlantic Ocean, and $L_{x}=148^{\circ}$ for the wider basin, giving a basin area close to that of the Indo-Pacific Ocean, 2.6 times wider than the narrow basin. The latitudinal extent of the model is $70^{\circ} \mathrm{S}$ to $70^{\circ}$ N. A Scotia Arc-like ridge is placed in the southern channel and shown in Figure 2-2 to provide a topographic drag and reduce the flow in the channel to realistic values Nadeau and Straub, 2009. The closures for subgrid-scale turbulence are standard and described in the Supplementary Information in Appendix A.

We use a linear equation of state with the same $\alpha$ and $\beta$ values as the analytical model. The boundary conditions are free-slip at the bottom and no-slip along the side walls. A sea ice package is used to produce the required buoyancy flux over the channel Jansen and Nadeau [2016]. The surface heat flux is computed from bulk formulas, as described in Jansen 2017] and in the Supporting Information, which are essentially a restoring boundary condition in sea-ice free regions. We prescribe the evaporation minus precipitation (E-P) as a freshwater flux given by:

$$
E-P=E_{0} f(y)
$$

where $f(y)$ is a function of latitude with units of $\mathrm{m} \mathrm{s}^{-1}$ and a magnitude corresponding to present day freshwater fluxes as shown in Figure 2-2 b. The function $f(y)$ stays the same in all simulations while we vary the magnitude of the freshwater flux through $E_{0} . E_{0}=1$ 

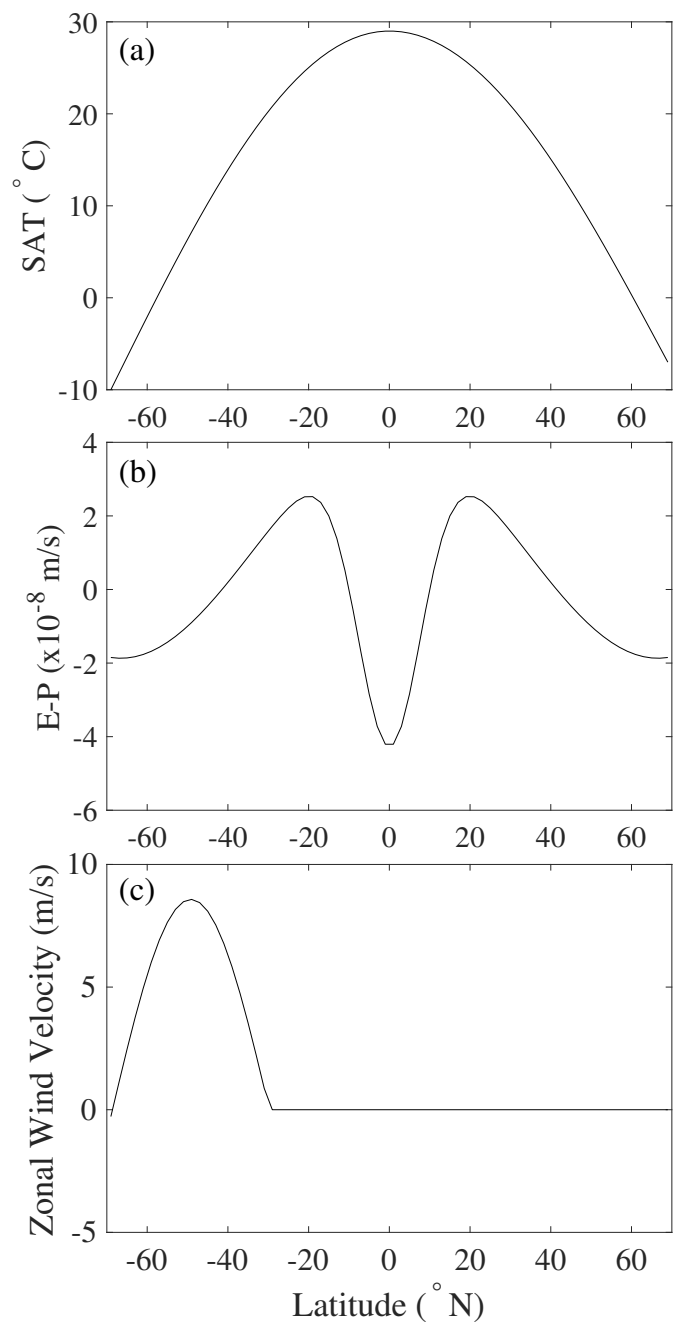

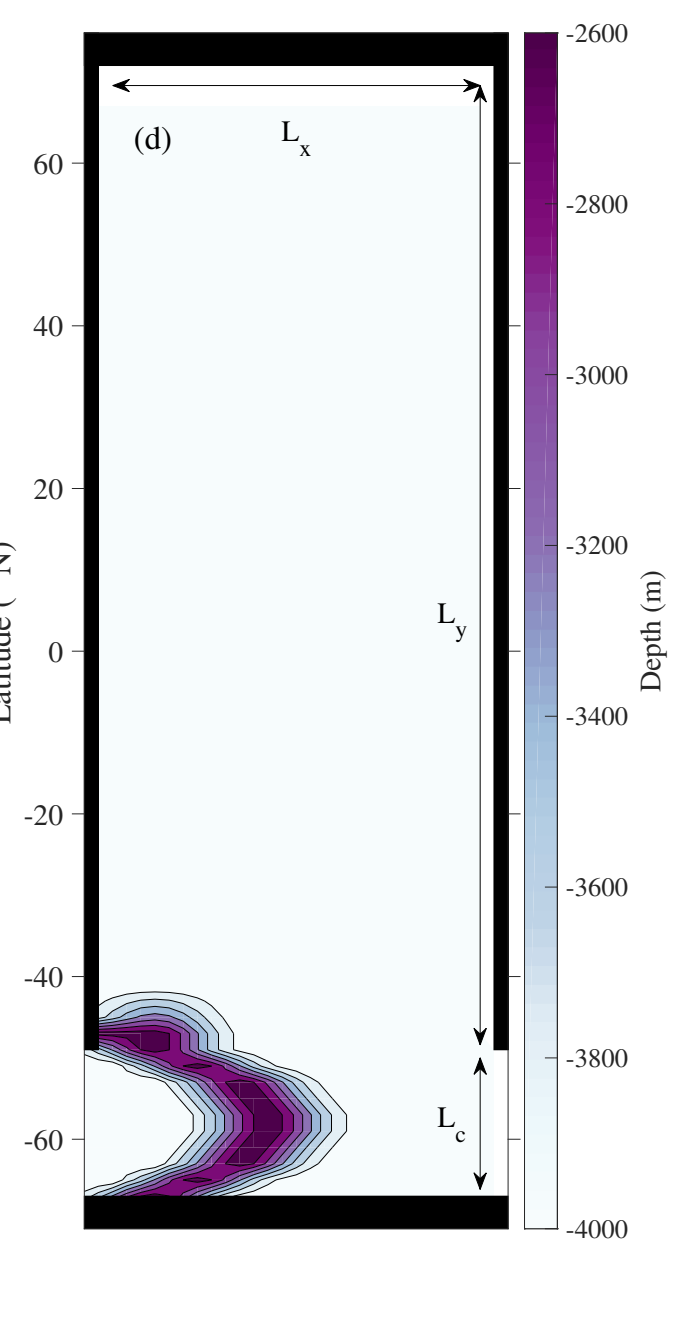

Figure 2-2: Surface forcing and geometry for the primitive equation model. All forcing functions vary only in latitude and not in longitude. (a) The prescribed surface air temperature used to compute air-sea heat fluxes. (b) The reference freshwater flux (E-P, evaporation precipitation) for $E_{0}=1$ in equation (2.8). The magnitude of the freshwater flux is varied across simulations by changing the value of $E_{0}$. (c) A wind stress over the southern re-entrant channel is computed from the zonal wind. (d) The model geometry. We run experiments with two widths $\left(L_{x}\right)$, a narrow Atlantic-like basin, and a wide Pacific-like basin. We included a Scotia-Arc like topographic feature in the channel.

corresponds approximately to the present-day zonally-averaged E-P values as diagnosed from the ECCO product Forget et al. 2015]. We apply a zonal wind stress only over the latitudes of the southern channel, which is imposed as an atmospheric zonal wind from which the EXF package Large and Pond, 2002 computes the surface stress (Figure 2-2.).

We report results from experiments with the two different basin widths. All experiments start with $E_{0}=0$ and are run to equilibrium for 17,000 years, then $E_{0}$ is increased smoothly 
at a rate of $0.05 E_{0}$ every 4,000 years. Since the freshwater flux is changed so slowly, the simulations are in quasi-steady state for almost all times. We kept increasing $E_{0}$ until convection shut off in the northern hemisphere. All diagnostics are computed from averages over the last 1,000 simulation years.

\subsubsection{Results}

Based on the two-layer model analysis, we anticipate a stronger salinity gradient in the wider basin than in the narrow basin. Fig. 2-3b,c shows that while the surface salinity patterns are similar in the narrow and wide basins, the depth-averaged salinity difference between low and high latitudes is much larger in the wider basin for the same freshwater forcing (Fig. 2-3 a). The increase in salinity gradient is not strictly monotonic for the narrow basin due to self-sustained oscillations that develop in the overturning strength at values of $E_{0}$ just above 1. A similar finding is discussed in Wolfe and Cessi 2015.

Fig. 2-4a shows the strength of the overturning circulation quantified as the zonallyaveraged maximum stream function at the northern wall, for simulations using different $E_{0}$ values. The overturning shuts off in the wide basin at a lower $E_{0 \text { crit }} \simeq 0.65$ than in the narrow basin where $E_{0 c r i t} \simeq 2$. Only the narrow basin convects at the "present day" freshwater forcing. The overturning is approximately twice as strong in the wide basin compared to the narrow basin, even though the wider basin is 2.6 times wider. This is consistent with the analytical model prediction that the difference in $E_{0 \text { crit }}$ stems from the fact that $\psi_{n}$ and the freshwater flux integrated over the whole basin scale differently with basin width.

Somewhat surprisingly the overturning strength does not decrease monotonously for increasing $E_{0}$ as predicted by the analytical model. In the narrow basin simulations the overturning slightly increases with $E_{0}$, while in the wide basin ones changes are small and non monotonic. The behavior is driven by increases in the adiabatic upwelling in the southern channel $\psi_{s}$ through physics we ignored in the analytical model. As can be seen in Fig. 2-3b,c, the salinity gradient increases more rapidly in the southern than in the northern hemisphere for increasing $E_{0}$, especially in the narrow basin. That's because buoyancy-driven gyres develop in the northern hemisphere (but not in the channel which has no lateral walls) which contribute additional salt transport to high latitudes. This asymmetry reduces the buoyancy gradient across the southern channel compared to the northern hemisphere. As a 


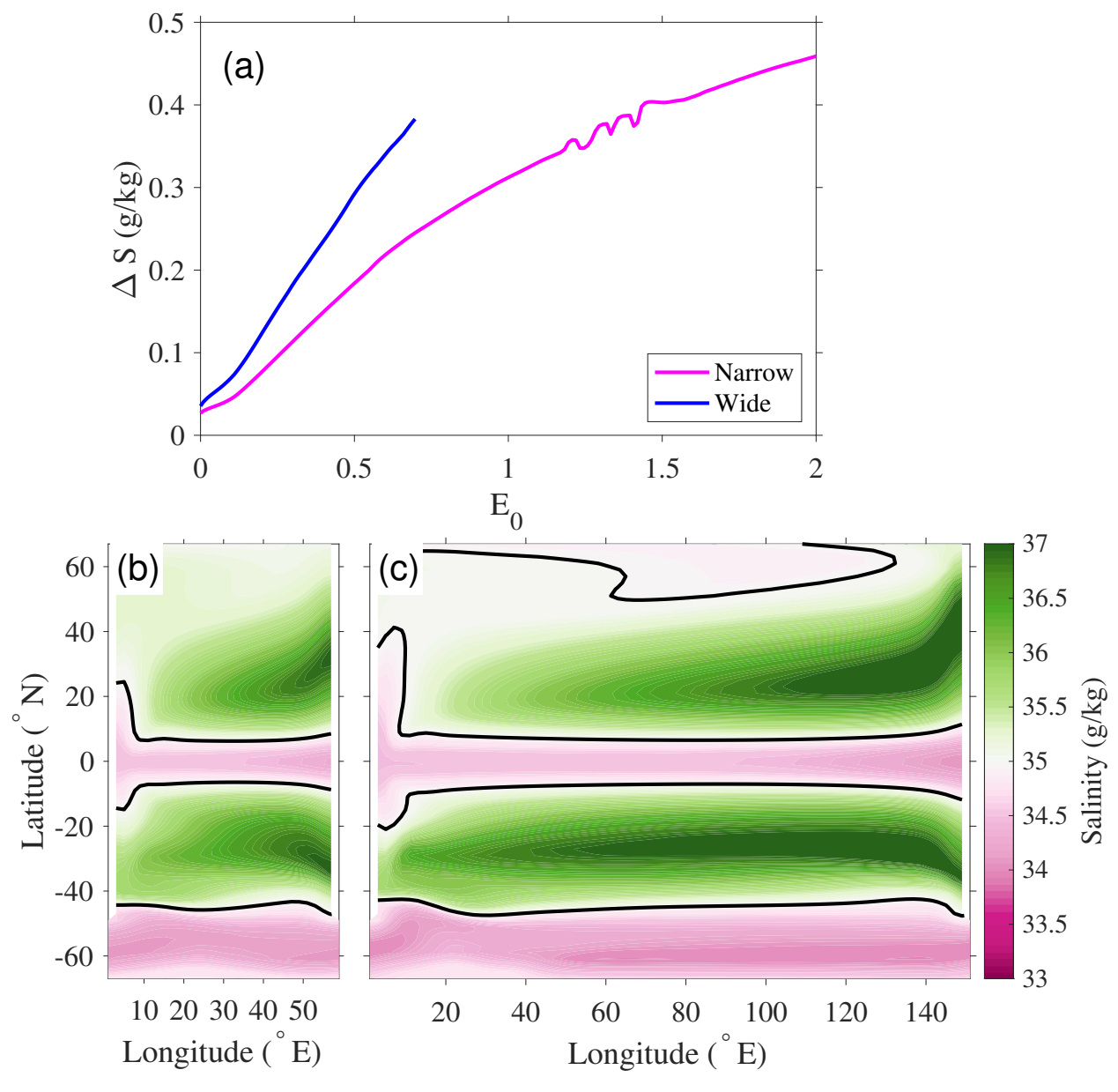

Figure 2-3: The wider basin shows a stronger salinity gradient in the MITgcm simulations. Panel (a) shows the difference in salinity between the northern boundary and 27 degrees north depth-averaged salinity for the narrow and wide basin simulations. The lines stop for the $E_{0 \text { crit }}$ at which convection shuts off. Panels (b) and (c) show the the surface salinity in the narrow (b) and wide (c) basins for a simulation run with $E_{0}=0.5$.

result the dense waters of the deep layer outcrop further south in the channel and thus the isopycnal slope decreases. A reduction in isopycnal slope $h / \ell$ is associated with an increase in $\psi_{s}$ as predicted by the analytical scaling in Eq. (2.3). While the analytical model could be extended to include these effects by explicitly adding an equation for $\ell$, such embellishments would not affect the key result that $\psi_{u}$ and $\psi_{s}$ have a different dependence on basin size than $\psi_{n}$. It is this difference that results in the dependence of $E_{0 c r i t}$ on basin size.

The numerical model has a somewhat different bifurcation: it is more like a supercritical bifurcation (Fig. 2-4 a). The full model has more flexibility in adjusting the stratification and the southern channel; nevertheless, it shows the same rapid shutdown and the non-existence 

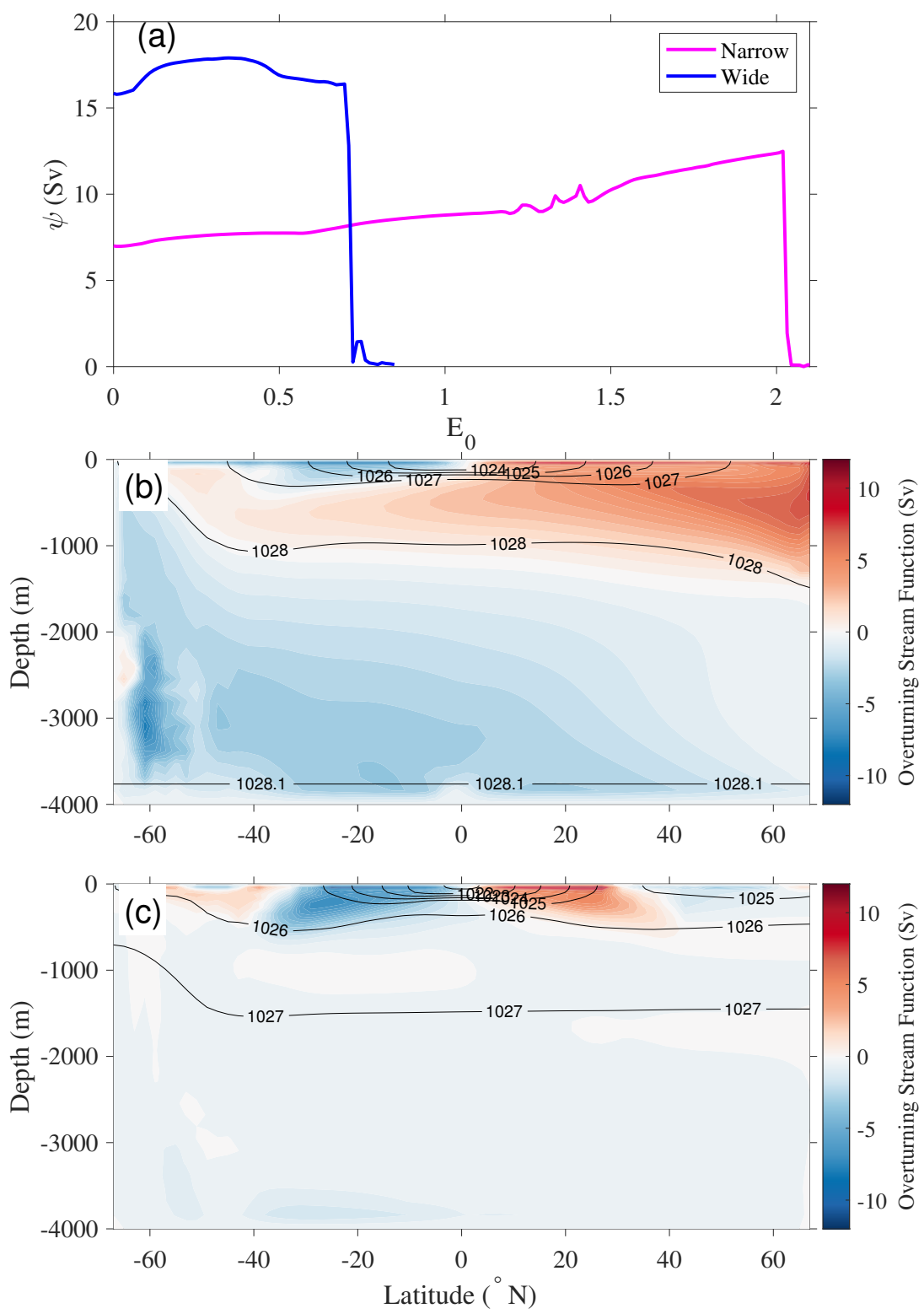

Figure 2-4: MITgcm simulations show that convection shuts off for a lower freshwater forcing $E_{0 \text { crit }}$ in the wider basin. Panel (a) shows overturning versus freshwater forcing for the narrow and wide basin. Panels (b,c) show overturning in the narrow basin before (b) and after convection shut off (c).

of a strong $\psi$ above $E_{0 c r i t}$.

\subsection{Discussion and Conclusions}

An extension of the two-layer analytical model developed by Gnanadesikan 1999 was used to compute the critical freshwater forcing at which high latitude convection shuts-off in a 
single-basin ocean connected to a re-entrant channel. We found that the wider the basin, the smaller the value of the critical freshwater forcing (Fig. 2-5). The dependence on basin size arises because the salinity difference between low and high latitudes, which counteracts the temperature difference's effect on density and thus suppresses high latitude convection, is higher in a wider basin. The salinity difference is set by the ratio of the freshwater flux into the ocean, which observations suggest is approximately constant per unit area Forget et al., 2015], and inversely proportional to the overturning streamfunction, which established scalings show that it is larger per unit area in a narrower basin. Idealized singlebasin simulations agree with the analytical model predictions that convection shuts off for weaker freshwater forcing in a wider basin. For present day freshwater forcing, our theory correctly predicts that convection is suppressed in a basin as wide as the North Pacific, but not in a basin as wide as the North Atlantic.
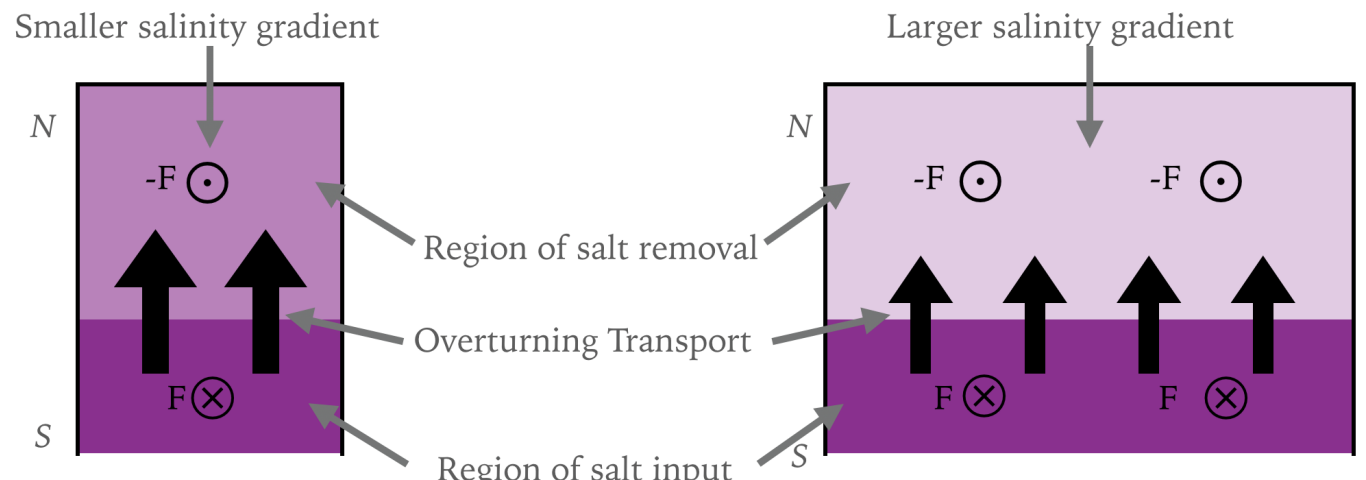

Figure 2-5: With a wider basin, the overturning is weaker per unit width, but the freshwater fluxes are the same per unit width, leading to a larger salinity gradient and a fresher northern part of the basin. This leads to a less dense convection region, and convection shut-off with weaker freshwater forcing.

Many plausible arguments have been advanced for why convection is observed only in the North Atlantic Ferreira et al. [2018]. Our work finds that scalings for the meridional overturning circulation favors convection in a smaller basin. In the ocean all these mechanisms are likely to be at play and their relative importance may differ in varied climates and continental configurations. By providing scaling laws for each contribution, we are in a better position to quantify each one and determine which is most important in any specific climate.

Future work will consider more realistic simulations of the ocean circulation and quan- 
tify the relative importance of the various mechanisms that favors convection in the North Atlantic. It is also possible that the mechanisms are not independent of each other and the interactions could play an important role in past climate transitions and future climate changes. Our work should not be taken as a theory for why deep convection is observed in the North Atlantic but not in the North Pacific. Rather we contribute new insight to this fundamental climate question. It seems that two fluid layers, a salt budget, and well established overturning scalings are all that is required to demonstrate the importance of basin width on high-latitude convection. 


\section{Chapter 3}

\section{Role of Residual Overturning for the}

Sensitivity of Southern Ocean

\section{Isopycnal Slopes to Changes in Wind Forcing}

(C)American Meteorological Society. Use with permission. 1

\subsection{Introduction}

Southern Ocean dynamics play a central role in the global ventilation of carbon and heat uptake and therefore in setting the global climate. The Southern Ocean has two counterrotating residual overturning cells of about $\sim 10 \mathrm{~Sv}$ each Lumpkin and Speer, 2007 forced by surface buoyancy fluxes that transfer tracers from the deep to the surface along isopycnals. In particular, the isopycnal slopes, or equivalently the thermal wind transport, are important for setting the outcropping location of the water masses (with different tracer concentrations) in the Southern Ocean The isopycnal slopes are also key for determining the depth of the global pycnocline Gnanadesikan, 1999, Nikurashin and Vallis, 2011. The Southern Ocean, forced by a strong wind, possesses a strong circumpolar current that is influenced by topography, notably from blocking ridges such as the Scotia Ridge and the

${ }^{1}$ Youngs et al. 2019 


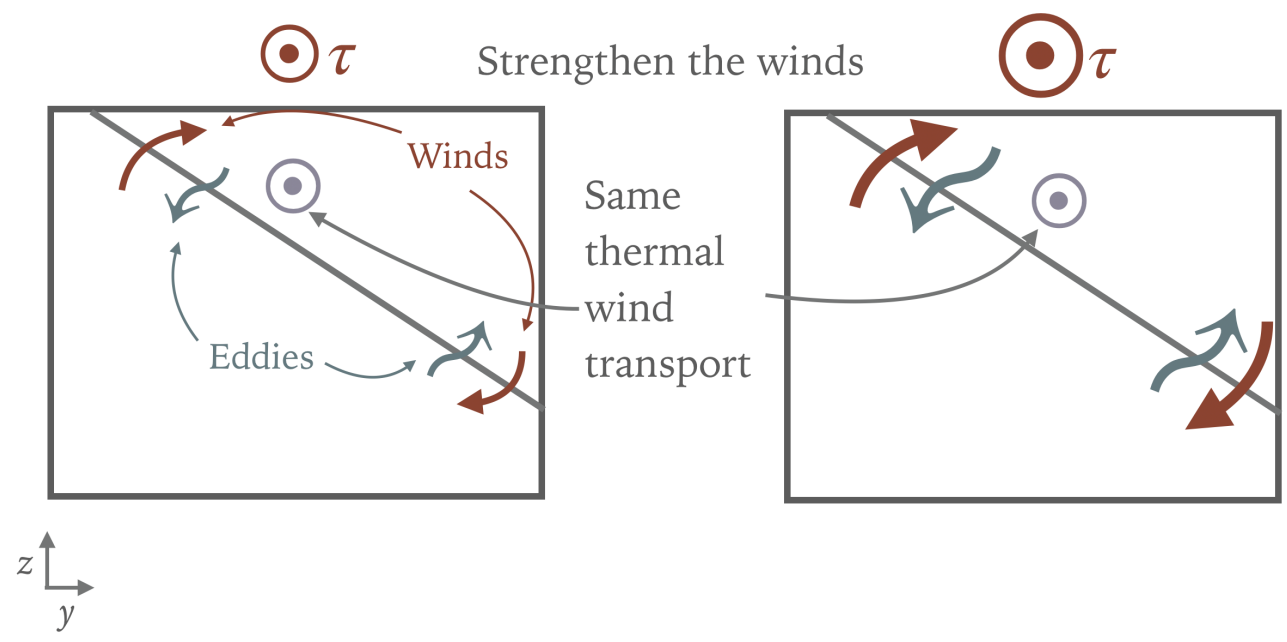

Figure 3-1: A schematic describing eddy saturation in the Antarctic Circumpolar Current. When a current is saturated, a change in wind leads to no change in the isopycnal slopes or thermal wind transport.

Kerguelen Plateau. An analysis of wind data from NCEP and ERA-Interim shows that the maximum zonally averaged wind stress over 1979-2016 is $0.14 \mathrm{~N} \mathrm{~m}^{-2}$ with a strengthening trend Lin et al., 2018; Thompson and Solomon, 2002, Marshall, 2003, Farneti et al., 2015]. It is an open question how the Southern Ocean circulation varies with changing wind, and in particular how sensitive the isopycnal slopes are to changing winds with a residual overturning.

In the Southern Ocean, isopycnal slopes are thought to arise from a balance of wind acting to steepen isopycnals and baroclinic eddies acting to flatten them Johnson and Bryden, 1989; Marshall and Radko, 2003. Theory suggests a connection between the isopycnal slopes and the baroclinic eddies, such that, as the wind forcing increases, the eddy fluxes will in kind increase due to increased eddy activity from baroclinic eddies that might be associated with a larger available potential energy reservoir Danabasoglu et al., 1994; Marshall, 1997]. This idea has been borne out qualitatively in some observations on short time scales [e.g. Meredith and Hogg, 2006, Hogg et al., 2015b], in equilibrium in idealized models [e.g. Munday et al., 2013, and on short time scales in a global model Bishop et al., 2016. This increase in eddy fluxes has led to the hypothesis of "eddy saturation": the isopycnal slopes in the Southern Ocean do not change with increasing wind forcing because eddies become strong enough to counter any increase in wind stress [e.g. Straub, 1993; Hallberg and Gnanadesikan, 2001] (Fig. 3-1). Several modeling studies have been found to be consistent with eddy saturation 
[e.g. Hallberg and Gnanadesikan, 2001, Munday et al., 2013, Bishop et al., 2016, but more work is still needed to fully understand under what conditions eddy saturation is realized.

There are several recent hypotheses to explain eddy saturation. One hypothesis suggests that the Southern Ocean is in an equilibrated state where there is an energy balance between eddy energy generation (which depends on isopycnal slopes and eddy energy through an Eady growth rate-like relationship) and the energy decay (which depends on the eddy energy), leading a change in wind stress to increase both the energy generation and decay in equal amounts when certain key parameters, such as drag coefficients, are fixed. The result is a constant transport Marshall et al., 2017; Mak et al., 2017]. Several other theories involve topography. Topography is likely a key aspect of eddy saturation because eddy fluxes tend to peak downstream of major topographic features [e.g. Thompson and Naveira Garabato, 2014; Youngs et al. 2017, Barthel et al., 2017. One hypothesis is that the topography increases the eddy efficiency, so as the wind stress changes the eddy activity changes without the slope needing to change very much to balance the winds Abernathey and Cessi, 2014. MacCready and Rhines, 2001]. Another theory focuses on the development of gyre circulations downstream of topography; for stronger winds, the barotropic gyres spin up, leaving the thermal wind transport unchanged Nadeau and Ferrari, 2015. Relatedly, Thompson and Naveira Garabato [2014] suggest that it is the flexing of standing meanders downstream of topography that accounts for saturation. Constantinou and Young 2017 and Constantinou [2018] suggest that baroclinic eddies are not even necessary for saturation, invoking a barotropic-topographic instability, albeit in an extreme parameter regime. All of these theories make progress, but do not take into account there is a residual overturning.

Few studies have connected the Southern Ocean isopycnal slopes and the idea of eddy saturation with the residual overturning present in the system 2 . There have been a couple of papers that examine a Southern Ocean-like system with wind forcing, topography, and a residual overturning in idealized frameworks, highlighting the importance of buoyancy forcing on the isopycnal slopes [e.g. Gnanadesikan and Hallberg, 2000, Hallberg and Gnanadesikan, 2001; Hogg, 2010; Morrison et al., 2011; Shakespeare and Hogg, 2012; Howard et al., 2015. Hallberg and Gnanadesikan 2001 use a two-layer primitive equation system with a positive residual overturning forced by an interface relaxation, wind stress, and bot-

\footnotetext{
${ }^{2}$ Here we are highlighting that there have only been a few studies that connect the idea of eddy saturation with the residual overturning, in part because of the increased complexity of a system with a residual overturning.
} 
tom topography to develop a scaling for the thermal wind transport when the system is dominated by the diabatic forcing, but do not examine the full parameter space, including a lower cell with a negative overturning. Hogg 2010 builds upon Hallberg and Gnanadesikan 2001 to examine a Southern Ocean-like system with a primitive equation model with both buoyancy and wind forcing and find that the system is wind-saturated. Howard et al. 2015] examine how the spin-up process of a channel differs with wind and buoyancy forcing. Many of these studies have examined only a limited wind- and buoyancy-forcing parameter space, so the validity of eddy saturation over a wide parameter space and the role of buoyancy forcing in modifying how the system responds to winds have not been fully explored.

Here we consider a broad range of forcings to try to gain a more complete picture of the physics of eddy saturation, building upon the work of Hogg [2010] and Howard et al. [2015] to test the theory of saturation with a larger forcing parameter space. We will address how the presence of a residual overturning modifies the response of isopycnal slopes to changing wind forcing.

We use a two-layer quasi-geostrophic re-entrant channel model with a flat bottom or with a ridge that blocks $f / h$ contours (Fig. 3-2 . The use of simplified models has a long history in being utilized to enhance our understanding of the Southern Ocean [e.g. McWilliams et al. 1978; Hallberg and Gnanadesikan, 2001]. This particular configuration is a simplified step on the way to understanding the full complexity of the Southern Ocean. At moderate wind stresses, the isopycnal slopes either steepen or flatten with an increasing wind stress, depending on the sign of the residual overturning, until the isopycnal slopes all approach a common value at strong wind stresses. The presence of the residual overturning increases the sensitivity of the isopycnal slopes to changes in wind forcing in the examined range of wind stresses. In section 2, we describe the model configuration and the budgets used for analysis. The results are described in section 3, and we provide discussions and conclusions in section 4 . 


\subsection{A 2-Layer Quasi-Geostrophic Analogue of the Southern Ocean}

\subsubsection{Model Configuration}

The two-layer quasi-geostrophic (QG) channel model we use as our analogue to the Southern Ocean (Fig. 3-2) is that described in Flierl and Pedlosky 2007): it solves for the mean zonal flow, the residual meridional overturning (combining the ageostrophic mean and the eddy contribution as defined below), and the eddy fields. These elements appear in many previous papers [e.g. Shepherd, 1983], however, we present a derivation from the full shallow water, two-layer system in the Appendix, showing that the appropriate definition of the residual vector velocity results in very similar equations.

Table 3.1: Table showing various parameters used for two-layer quasi-geostrophic model.

\begin{tabular}{c|l|l}
\hline Parameter & Description & value \\
\hline$\mu$ & Bottom drag & $3.47 \times 10^{-7} \mathrm{~s}^{-1}$ \\
$r_{d}$ & Deformation radius & $15 \mathrm{~km}$ \\
$H_{1}$ & Upper layer thickness & $2 / 3 \mathrm{~km}$ \\
$H_{2}$ & Lower layer thickness & $31 / 3 \mathrm{~km}$ \\
$\kappa$ & Potential vorticity diffusivity & $15 \mathrm{~m}^{2} \mathrm{~s}-1$ \\
$f_{0}$ & Coriolis frequency & $-1 \times 10^{-4} \mathrm{~s}^{-1}$ \\
$\beta$ & Meridional derivative of Coriolis frequency & $1 \times 10^{-11} \mathrm{~m}^{-1} \mathrm{~s}^{-1}$ \\
$\mathrm{dx}$ & Grid size & $15.625 \mathrm{~km}^{-3}$ \\
$\rho_{0}$ & Reference density & $1000 \mathrm{~kg} \mathrm{~m}^{-3}$ \\
\hline
\end{tabular}

Model Parameters Our channel has free-slip walls at $y= \pm L / 2$ with $L=2000 \mathrm{~km}$ and periodic conditions at $x=0$ and $x=4000 \mathrm{~km}$ (Table 1). The upper layer has thickness $h_{1}=H_{1}+h$ with the mean value $H_{1}=2 / 3 \mathrm{~km}$; the lower layer has $h_{2}=H_{2}-h-h_{b}(x)$ and $H_{2}=31 / 3 \mathrm{~km}$. We picked the relative layer thicknesses based on the stratification as observed in the Southern Ocean Karsten and Marshall, 2002. The reduced gravity $\left(g^{\prime}\right)$ is chosen such that the deformation radius is $15 \mathrm{~km} 3$, based on an estimation of the value for much of the Southern Ocean Chelton et al., 1998 and a reference density $\rho_{0}=1000 \mathrm{~kg}$ $\mathrm{m}^{-3}$, used later for scaling. The grid size is around $15 \mathrm{~km}$, and, even though it is around the

\footnotetext{
${ }^{3}$ Here the actual value of $g^{\prime}$ is not important because it is never an independent parameter but only appears as part of the deformation radius.
} 


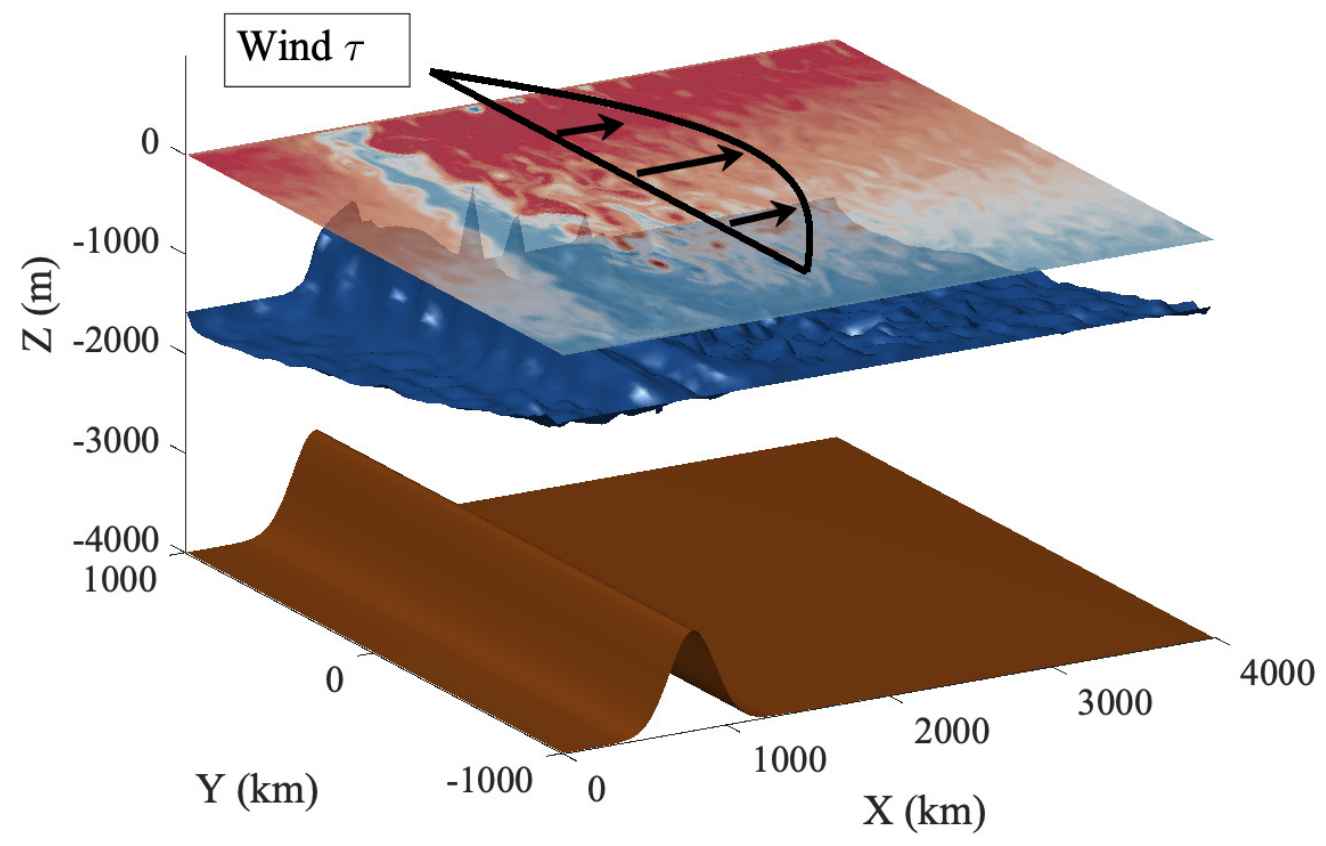

Figure 3-2: A three-dimensional figure showing the model domain. The color on the surface is a snapshot of the upper-layer potential vorticity for the case with a wind stress maximum $\tau_{0}=0.2 \mathrm{~N} \mathrm{~m}^{-2}$ and residual overturning $\mathcal{H}_{0}=-2 \mathrm{~Sv}$. The blue surface represents the instantaneous interface height. The wind forcing is shown above the surface.

same size as the deformation radius $r_{d}$, we expect the dynamics will be adequately resolved since the wavelength of the fastest growing baroclinic instability wave is order $10 r_{d}$ and the nonlinear motions tend to be several times larger than $r_{d}$ Pedlosky, 1987. Additionally, we have run several key simulations at twice the resolution which lead to the exact same conclusions concerning the response of the thermal wind transport to the winds and less than a $10 \%$ difference in the thermal wind transport. Non-conservative terms are wind stress, $\tau$, a linear bottom drag with coefficient $\mu$, and buoyancy forcing, $\mathcal{H}$, that represents warming which transforms lower layer water to upper layer water or cooling which does the opposite. We also include a small down-gradient potential vorticity flux with diffusivity $\kappa$ to remove small-scale enstrophy. See Table 1 for parameter values.

Topography The elevation of the topographic ridge, $h_{b}(x)$, varies only zonally. We have performed simulations with two different topographies, a flat bottom $h_{b}=0 \mathrm{~m}$ and a simple 
cross channel Gaussian ridge centered at $x=x_{0}$ :

$$
h_{b}(x)=h_{0} e^{-\left(x-x_{0}\right)^{2} / \sigma^{2}}
$$

where $h_{0}=1000 \mathrm{~m}, \sigma=200 \mathrm{~km}, x_{0}=800 \mathrm{~km}$. The total depth away from the topography is $4000 \mathrm{~m}$, and the ridge is $30 \%$ of the lower layer thickness. We also ran simulations with $h_{0}=2000 \mathrm{~m}$ for verification and find that the thermal wind transport saturates to the same value but reaches the saturation at a smaller wind stress. However, we focus on $h_{0}=1000$ $\mathrm{m}$ because the smaller ridge is more consistent with the quasi-geostrophic assumptions.

Residual Formulation To recast the mass equation in our residual flow formulation, we split the mass transport within a layer into a rotational and divergent part Kushner and Held, 2002]:

$$
\mathbf{u}_{i}^{*}=\mathbf{u}_{i} \frac{h_{i}}{H_{i}}-\hat{\mathbf{z}} \times \nabla \psi_{i} \quad \Rightarrow \quad \nabla \cdot\left(\mathbf{u}_{i} h_{i}\right)=H_{i} \nabla \cdot \mathbf{u}_{i}^{*}
$$

where $\psi_{i}$ is the rotational component of the horizontal velocities $\mathbf{u}, \nabla$ represents a horizontal gradient and ()$^{*}$ represents the residual component. The zonally averaged mass equation becomes linear:

$$
\frac{\partial}{\partial t}\left\langle h_{1}\right\rangle+\frac{\partial}{\partial y}\left\langle h_{1} v_{1}\right\rangle=\frac{\partial}{\partial t}\left\langle h_{1}\right\rangle+H_{1} \frac{\partial}{\partial y}\left\langle v_{1}^{*}\right\rangle=\langle\mathcal{H}\rangle \quad \text { or } \quad \frac{\partial}{\partial t}\left\langle h_{1}\right\rangle+\left\langle w^{*}\right\rangle=\langle\mathcal{H}\rangle,
$$

where the angle brackets denote a zonal average. The residual vertical velocity, $w^{*}=$ $w^{a}+\mathbf{u}_{1} \cdot \nabla h_{1}$ relates the cross-interface flow to the two components of the up- or down-slope velocity, with $w^{a}$ being the actual vertical velocity including that associated with along-slope motions (with the superscript emphasizing that the velocity is ageostrophic). Equation 3.1 shows not only the link between the residual overturning $H_{1}\left\langle v^{*}\right\rangle=\left\langle h_{1} v_{1}\right\rangle$ and the buoyancy forcing but also the way in which the residual flow encompasses both the ageostrophic and eddy fluxes, with the two on average cancelling when $\mathcal{H}=0$ and, in general, partially offsetting each other. Note that the displacement of the top surface can be neglected in the mass equation so that $\partial h_{2} / \partial t=-\partial h_{1} / \partial t$; this implies that $H_{1}\left\langle v_{1}^{*}\right\rangle+H_{2}\left\langle v_{2}^{*}\right\rangle=0$, and we only need to find the residual circulation in the upper layer.

Equations of Motion As detailed in the Appendix, using the definitions above for $\mathbf{u}_{i}^{*}$ and $w^{*}$, supplemented with $p_{i}^{*}=p_{i}-f_{0} \psi_{i}+\left|\mathbf{u}_{i}\right|^{2} / 2$, and then making the usual small Rossby 
number expansion leads to the quasi-geostrophic equations in the two layers Pedlosky, 1987):

$$
\frac{\partial}{\partial t} q_{i}+\mathbf{u}_{i} \cdot \nabla q_{i}= \pm f_{0} \frac{\mathcal{H}}{H_{i}}-\delta_{i 1} \frac{\tau_{y}}{\rho_{0} H_{1}}+\kappa \nabla^{2} q_{i}-\delta_{i 2} \mu \nabla^{2} \psi_{i}
$$

The potential vorticity is $q_{i}=f_{0}+\beta y+\delta_{i 2} f_{0} h_{b} / H_{2}+\nabla^{2} \psi_{i} \mp F_{i}\left(\psi_{1}-\psi_{2}\right)$, and $F_{i}=f_{0}^{2} / g^{\prime} H_{i}$ are the stratification parameters, which are related to the deformation radius $r_{d}$ by $F_{1}+F_{2}=$ $1 / r_{d}^{2}$. We have included a small diffusivity $\kappa$ which is generally negligible unless there are no eddies to transport energy vertically downwards where it can be dissipated by bottom drag.

It is useful both computationally and for diagnostics to split out the zonal mean flow and advance $\left\langle u_{i}\right\rangle$ with the zonally-averaged momentum equation

$$
\frac{\partial}{\partial t}\left\langle u_{i}\right\rangle-f_{0}\left\langle v_{i}^{*}\right\rangle-\left\langle v_{i}^{\prime} q_{i}^{\prime}\right\rangle=\delta_{i 1} \frac{\tau}{\rho_{0} H_{1}}-\delta_{i 2} \mu\left\langle u_{2}\right\rangle
$$

where primes represent a deviation from the zonal average. We can use the thermal wind equation,

$$
f_{0}\left(\left\langle u_{1}\right\rangle-\left\langle u_{2}\right\rangle\right)=g^{\prime} \partial h_{1} / \partial y
$$

to find a diagnostic equation for $\left\langle v_{1}^{*}\right\rangle$

$$
\left[\frac{\partial^{2}}{\partial y^{2}}-F_{1}-F_{2}\right]\left\langle v_{1}^{*}\right\rangle=\frac{f_{0}}{g^{\prime} H_{1}}\left[\left\langle v_{1}^{\prime} q_{1}^{\prime}\right\rangle-\left\langle v_{2}^{\prime} q_{2}^{\prime}\right\rangle+\frac{\tau}{\rho_{0} H_{1}}+\mu\left\langle u_{2}\right\rangle\right]+\frac{1}{H_{1}} \frac{\partial}{\partial y}\langle\mathcal{H}\rangle .
$$

The zonally varying QG equations

$$
\frac{\partial}{\partial t} q_{i}^{\prime}+\left\langle u_{i}\right\rangle \frac{\partial}{\partial x} q_{i}^{\prime}+\nabla \cdot\left(\mathbf{u}_{i}^{\prime} q_{i}^{\prime}\right)-\frac{\partial}{\partial y}\left\langle v_{i}^{\prime} q_{i}^{\prime}\right\rangle=\kappa \nabla^{2} q_{i}-\delta_{i 2} \mu \nabla^{2} \psi_{i}
$$

together with Equations 3.3 and 3.5 comprise our model equations.

Model Forcing The buoyancy forcing - cross-isopycnal mass transfer - is taken to be $\mathcal{H}=-\mathcal{H}_{0} \sin (\pi y / L)$. To put $\mathcal{H}_{0}$ in perspective, consider the upper layer mass equation

$$
\frac{\partial}{\partial t} h_{1}+\nabla \cdot\left(\mathbf{u}_{1} h_{1}\right)=\mathcal{H}
$$


which takes the form

$$
\frac{\partial}{\partial t}\left\langle h_{1}\right\rangle+\frac{\partial}{\partial y}\left\langle v_{1} h_{1}\right\rangle=\mathcal{H}
$$

after a zonal mean (indicated by angle brackets). Thus, the time-mean northward mass transport — the meridional overturning — is just

$$
\left\langle v_{1} h_{1}\right\rangle \equiv\left\langle v_{1}^{a}\right\rangle\left\langle h_{1}\right\rangle+\left\langle v_{1}^{\prime} h_{1}^{\prime}\right\rangle=\langle\mathcal{H}\rangle=H_{1}\left\langle v_{1}^{*}\right\rangle
$$

with $v^{a}$ the ageostrophic velocity (since the geostrophic northward velocity has zero zonal average). We choose $\mathcal{H}$ to drive a northward transport - the residual overturning - of 2 or $4 \mathrm{~Sv}$ because our channel is about $1 / 4$ the length of the Antarctic Circumpolar Current. Our cases would represent 8 and $16 \mathrm{~Sv}$ of overturning with a channel the length of the Antarctic Circumpolar Current (ACC) compared to the estimate $10 \mathrm{~Sv}$ in the Southern Ocean $4^{4}$ When we discuss positive overturning we mean that $\mathcal{H}_{0}>0$ and time-averaged $\left\langle h_{1} v_{1}\right\rangle>0$, so that there is sinking in the north and upwelling in the south; negative overturning has the opposite sense with upwelling in the north and sinking in the south.

We want to explore a range of wind and buoyancy forcings but identify those we consider to be "realistic" to assess the sensitivity of the isopycnal slopes to changes in forcing within a range that might conceivably apply to Southern Ocean. Given the idealizations of the model, we cannot expect precise matching. We assume wind stresses of the form $\tau=\tau_{0} \cos (\pi y / L)$ and vary $\tau_{0}$ between 0 and $0.8 \mathrm{~N} \mathrm{~m}^{-2}$. Observations Lin et al., 2018, Farneti et al., 2015 suggest $\tau_{0}$ is about $0.14 \mathrm{~N} \mathrm{~m}^{-2}$, so we will consider $\tau_{0}>0.2 \mathrm{~N} \mathrm{~m}^{-2}$ to be "strong" and less than $0.1 \mathrm{~N} \mathrm{~m}^{-2}$ "weak". To put these in a similar context to the buoyancy forcing, we look at the mean zonal momentum equations (Equation 3.3). If we multiply the upper layer equation by $H_{1} / f_{0}$, we can then compare the residual overturning transport to the eddy transport and wind-driven overturning. The scaled wind-stress term is just the Ekman transport $\tau / \rho_{0} f_{0} ; \tau_{0}=0.1 \mathrm{~N} \mathrm{~m}^{-2}$ would generate a $4 \mathrm{~Sv}$ transport, smaller values than this indicate weak wind transports. Ekman transports larger than $8 \mathrm{~Sv}$ would indicate large wind transports. Of course, in the absence of buoyancy forcing, this transport would be on average balanced by an opposite eddy mass flux. We note that wind stress curl is an important part of subduction and therefore meridional overturning in a fully stratified ocean

\footnotetext{
${ }^{4}$ Since the ACC traverses multiple topographic features, modeling a segment of it rather than a single ridge in a longer channel seems appropriate.
} 
Marshall and Speer, 2012; in our quasi-geostrophic setup, the "subduction" is fixed even though the wind stress curl is proportional to $\tau_{0}$.

Thermal Wind Transport We wish to diagnose the mean isopycnal slopes, so we utilize the relationship between the isopycnal slopes and the transport through the thermal wind relationship created by combining Equations 3.1 and 3.3 . We calculate the thermal wind transport

$$
T_{w}=H_{1} \int\left(\left\langle u_{1}\right\rangle-\left\langle u_{2}\right\rangle\right) d y
$$

i.e., the two-layer analogue of the transport calculated geostrophically, assuming a level of motion at the bottom, to highlight the connection between the transport and the isopycnal slopes. "Baroclinic transport" is often used for this quantity, but we prefer not to use this term since it is confusing when thinking in terms of vertical modes.

Important Limits All the simulations we discuss have an active eddy field; the simulations with a ridge also exhibit a large standing eddy or gyre downstream of the ridge, coincident with the most strongly eddying flow (Fig. 3-3). For a flat-bottomed situation, the lower layer potential vorticity gradient changes sign when the thermal wind transport reaches $18 \mathrm{~Sv}$, rendering the system susceptible to baroclinic instability. For the linear problem, we can calculate the forcing that first generates eddies. If there is just a residual overturning, that value is about $\mathcal{H}_{0}=-0.03 \mathrm{~Sv}$. If there is only a wind stress, a forcing

of about $\tau_{0}=10^{-3} \mathrm{~N} \mathrm{~m}^{-2}$ is sufficient to reverse the potential vorticity gradients. These particular values depend on potential vorticity diffusivity, $\kappa$. For almost the entire range of wind stresses and residual overturnings that we test, the system is vigorously eddying. We do not consider positive residual overturning and wind forcing combinations that have westward transport: the gradient of the Coriolis parameter $\beta$ imposes an asymmetry between eastward and westward flow and hence a westward flow behaves very differently and puts itself outside the parameter space that is relevant to the Southern Ocean.

\subsubsection{Connection between overturning and slope}

The common conceptual ideas of saturation usually involve steepening of the thermocline slope by increased wind being offset by baroclinic instability fluxing heat and acting to flatten the thermocline slope, making the thermal wind transport relatively insensitive to 
the strength of the wind forcing. However, it turns out that topography plays a crucial role in saturation, for example, by modifying the baroclinic activity Abernathey and Cessi, 2014]. Given the significant changes in the flow structure associated with topography or heat flux, it seems important to examine how the transport varies with an interface heat flux as well. The addition of a heat flux across the interface certainly modifies the isopycnal slope.

We will examine how in the following thought experiment. Consider the following: start with a system with no buoyancy forcing in equilibrium and consider the time-averaged circulations. The residual velocity is zero (Equation 3.1) and the upper layer momentum equation (Equation 3.3) implies that the wind stress is balanced by southward eddy potential vorticity fluxes. With eastward upper-layer flow $\left\langle u_{1}\right\rangle$, we expect from the thermal wind equation that the interface will be shallow at the south and deep at the north i.e. $g^{\prime} \partial\langle h\rangle / \partial y \sim-f_{0}\left\langle u_{1}\right\rangle>0$. When we add a buoyancy flux $\mathcal{H}_{0}<0$ such that there is cooling in the south and warming in the north, the interface will begin to tilt further. This is partially impeded by a southward residual flux $\left(\partial\left\langle v_{1}^{*}\right\rangle / \partial y\right.$, which is negative in the south and positive in the north). Equation 3.3 then shows that the eddy potential vorticity flux must become more negative to halt the steepening of the interface. This is consistent with enhanced baroclinic instability. But we are left with a net increase in the interface slope and the thermal-wind transport when the overturning is negative. Likewise, with a positive residual overturning $\left(\mathcal{H}_{0}>0\right)$, the interface slope will decrease compared to when there is no overturning (Fig. 3-4).

\subsubsection{Budgets}

Splitting out the zonal average provides as a useful framework for physical interpretation. The time-average (indicated by an overbar) of the upper layer momentum equation (3.3), taking advantage of $\tau$ depending only on $y$ results in

$$
\left\langle\overline{v_{1}^{\prime} q_{1}^{\prime}}\right\rangle+f_{0}\left\langle\overline{v_{1}^{*}}\right\rangle+\frac{\tau}{\rho_{0} H_{1}}=0
$$

and expresses the trade-off between wind stress, residual overturning, and eddy fluxes in increasing (if positive) or decreasing (if negative) the upper layer zonal flow. Residual 
overturning from the time-mean mass equation (Equation 3.1)

$$
H_{1} \frac{\partial}{\partial y}\left\langle\overline{v_{1}^{*}}\right\rangle=\mathcal{H}
$$

can be integrated in $y$ and yields our momentum balance diagnostic:

$$
\left.\overline{\left\langle v_{1}^{\prime} q_{1}^{\prime}\right.}\right\rangle+\frac{f_{0}}{H_{1}} \int_{0}^{y} \mathcal{H}\left(y^{\prime}\right) d y^{\prime}+\frac{\tau}{\rho_{0} H_{1}}=0
$$

or, for the specific forms of $\tau$ and $\mathcal{H}$ used,

$$
\left\langle\overline{v_{1}^{\prime} q_{1}^{\prime}}\right\rangle=-\frac{f_{0}}{H_{1}}\left[\frac{L}{\pi} \mathcal{H}_{0}+\frac{\tau_{0}}{\rho_{0} f_{0}}\right] \cos (\pi y / L)
$$

For $\tau_{0}>0$ and $f_{0}<0$, the eddy potential vorticity flux must be stronger (weaker) for negative (positive) overturning.

The upper-layer potential vorticity flux has a relative vorticity contribution $\left\langle\overline{\left\langle v_{1}^{\prime} \nabla^{2} \psi_{1}^{\prime}\right.}\right\rangle$ and a thickness flux $-f_{0} / H_{1}\left\langle\overline{v_{1}^{\prime} h^{\prime}}\right\rangle$. If we neglect the former, since it has no contribution in the domain integral and is generally small in our experiments, then the eddy thickness (or heat) flux is

$$
\left.\overline{\left\langle v_{1}^{\prime} h^{\prime}\right.}\right\rangle=\left[\mathcal{H}_{0}+\frac{\tau_{0}}{\rho_{0} f_{0}}\right] \cos (\pi y / L)
$$

Since the derivation of Equation (3.10) uses also the thickness and heat equations, we could instead think about this equation as a heat or thickness budget Plumb and Ferrari, 2005. In this heat budget, the wind stress term represents the mean advection of heat by the ageostrophic flow, and the eddy terms are the eddy advection of heat, and the interface forcing is the input of heat. Similarly, this balance could also be thought of as the different components of the residual overturning, where the total residual overturning given by the interface forcing is just the balance between the wind-driven overturning and eddy-driven overturning.

Summing the upper and lower layer momentum equations, weighted by the layer thicknesses equations gives the traditional momentum balance:

$$
\frac{\tau}{\rho_{0}}=f_{0}\left\langle h_{b} \bar{\psi}_{2 x}^{\prime}\right\rangle+\mu H_{2}\left\langle u_{2}\right\rangle
$$

where ${ }_{x}$ indicates a derivative with respect to $x$. For the simulations with a ridge, the form 

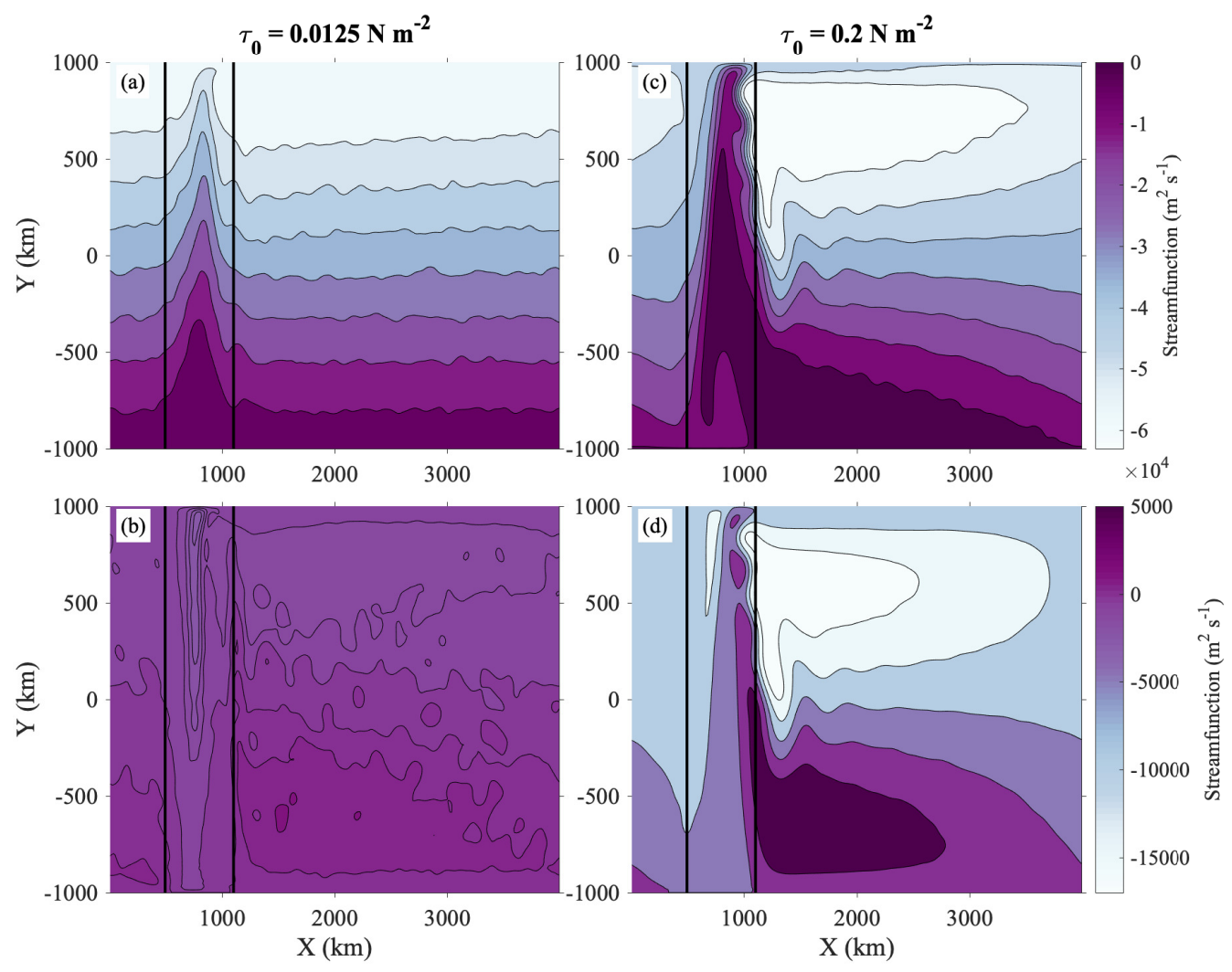

Figure 3-3: A figure showing the streamfunctions for the cases where residual overturning $\mathcal{H}_{0}=-2 \mathrm{~Sv}$ for $(\mathrm{a}, \mathrm{b})$ weak winds of $\tau_{0}=0.0125 \mathrm{~N} \mathrm{~m}^{-2}$ and $(\mathrm{c}, \mathrm{d})$ relatively strong winds of $\tau_{0}=0.2 \mathrm{~N} \mathrm{~m}^{-2}$. Panels (a,c) show the time averaged upper-layer transport, $\overline{\psi_{1}}$, panels $(\mathrm{b}, \mathrm{d})$ show the time averaged lower-layer transport, $\overline{\psi_{2}}$. The black lines indicate where the topography exceeds $100 \mathrm{~m}$.

drag $\left(f_{0}\left\langle h_{b} \bar{\psi}_{2 x}^{\prime}\right\rangle\right)$, from the difference in geostrophic pressure on either side of the topography, balances the momentum input, whereas for the flat bottom case, the momentum input by the wind is removed by bottom drag Munk and Palmén, 1951.

\subsection{Results}

We investigate two main questions: Where in the parameter space is the flow saturated, and what role does the residual overturning play in setting the thermal wind transport? The simulations performed have several different residual overturnings over a range of wind stresses (Fig. 3-2). We analyze the resulting thermal wind transport, heat fluxes, and form drag in order to understand how and why the isopycnal slopes vary over the wide range of forcings examined. 


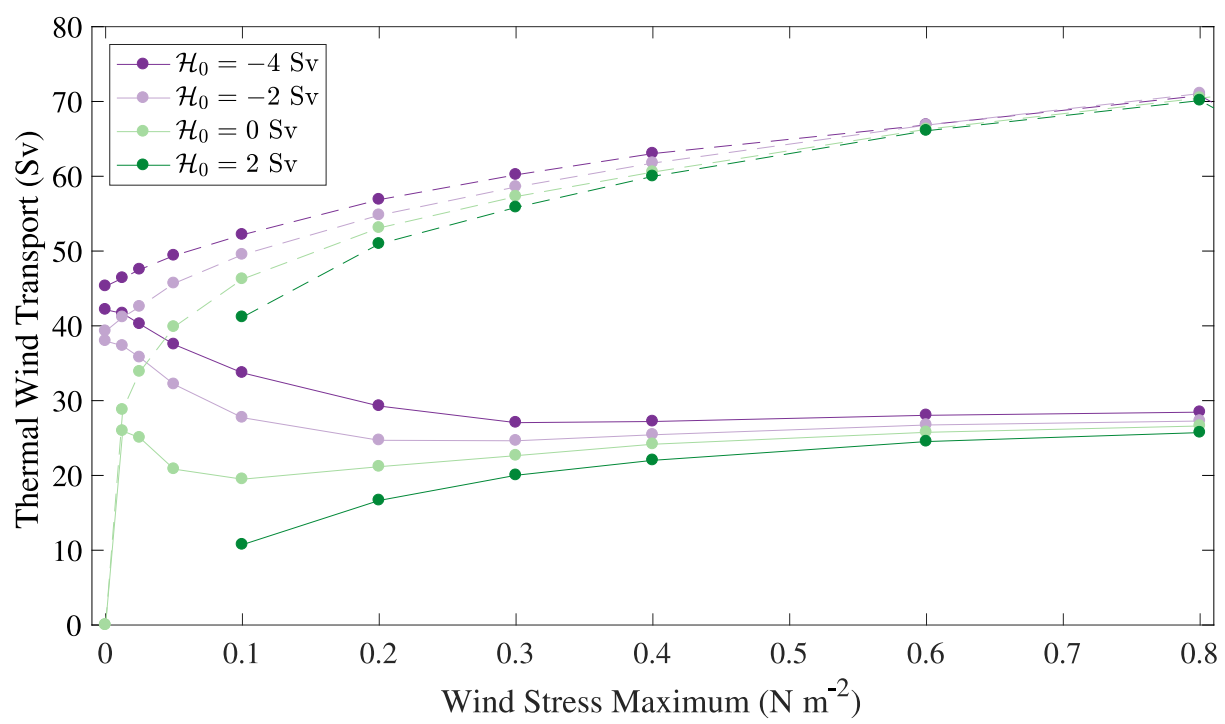

Figure 3-4: Thermal wind transport versus wind stress for four different overturnings with (solid) and without (dashed) a $1000 \mathrm{~m}$ tall ridge. At strong wind stresses there is saturation, but as the wind forcing weakens to moderate, present day, levels, the slopes change with the wind, in different directions depending on the sign of the overturning in order to approach the transport for a flat bottom. When the wind stress is very small and the overturning positive, the flow is westward and will not be discussed further.

\subsubsection{Strong wind forcing}

There is a history of studies that find saturation in a channel with a blocking ridge and no saturation with a flat-bottom [e.g. Munday et al., 2015; Nadeau and Ferrari, 2015]. Indeed, we see in Figure 3-4 that the thermal wind transport still changes with wind stress in the flat-bottomed simulations across the imposed wind stresses. By comparison, we see eddy saturation in the simulations with a ridge, but only at strong wind forcing, $\tau_{0}>0.2 \mathrm{~N} \mathrm{~m}^{-2}$. On the other hand, the vertically-integrated transport is essentially constant for wind stress $\tau_{0}<0.2 \mathrm{~N} \mathrm{~m}^{-2}$, but increases linearly with the wind stress when $\tau_{0}>0.2 \mathrm{~N} \mathrm{~m}^{-2}$ (not shown).

Since we see that the flow is saturated at strong wind forcing, we question what allows eddy saturation. We calculate the eddy heat fluxes from Equation 3.10 and normalize them by dividing by the total heat transport. We see that the normalized heat fluxes are concentrated into the meander regions when the wind forcing is strong and that in the downstream region the heat fluxes are irrelevant (Fig. 3-5 5. This result has also been seen in MacCready and Rhines 2001 and Abernathey and Cessi 2014. The meander

\footnotetext{
${ }^{5}$ Note here that the eddy fluxes downstream are in fact upgradient due to a decay of the eddy energy. We can see this also in Youngs et al. 2017.
} 

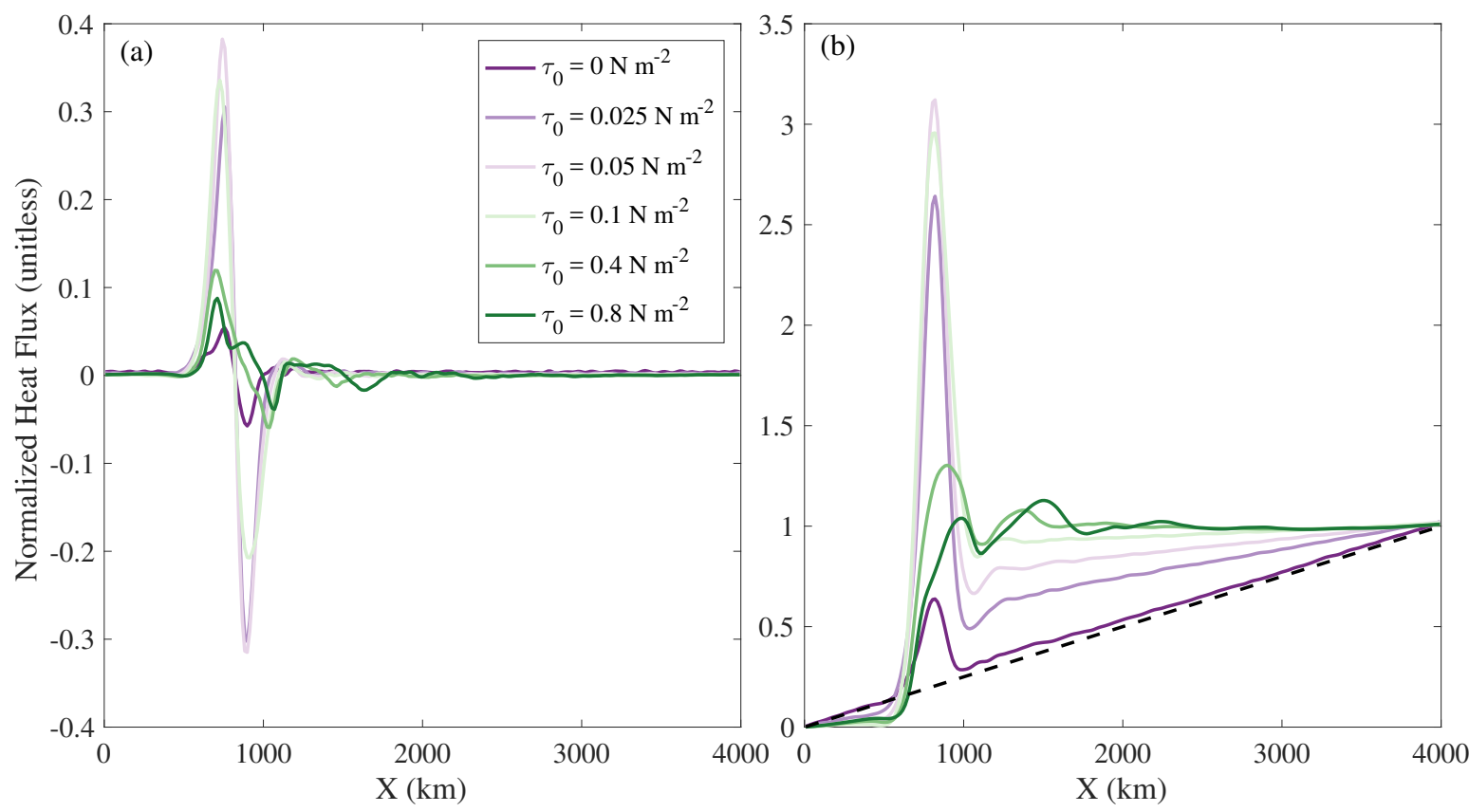

Figure 3-5: Panel (a) shows eddy heat fluxes normalized by the total heat fluxes along the channel for $\mathcal{H}_{0}=-2 \mathrm{~Sv}$. Most of the heat fluxes occur in or just downstream of the meander. Panel (b) shows the cumulative normalized eddy heat flux along $\mathrm{x}$, indicating that when the wind is strong, most of the heat fluxes occur in the meander, but with weaker winds, the heat fluxes throughout the whole domain are just as important. The black dashed line shows the heat fluxes for a flat-bottomed experiment.

strengthens the temperature gradients locally, leading to larger heat fluxes across mean streamlines. However, the gradients tend to be zonal (and opposite signed on either side of the ridge) instead of meridional, leading to very little change in the zonally-averaged isopycnal slopes. Overall, the total heat fluxes are increasing linearly with the wind, but are localized near the ridge, indicating that it is primarily the meander that is contributing to all of the changes in eddy fluxes. Thus, understanding the meander system is incredibly important for understanding the saturation process.

\subsubsection{Realistic Winds}

Outside of the strong wind forcing limit, i.e., for $\tau_{0}<0.2 \mathrm{~N} \mathrm{~m}^{-2}$, the thermal wind transport is more sensitive to changes in wind forcing (Fig. 3-4). In the Southern Ocean, a realistic mean wind stress range is about 0.1 to $0.2 \mathrm{~N} \mathrm{~m}^{-2}$ Lin et al. 2018. In this range, thermal wind transport either increases or decreases with the wind stress, depending on the sign of the overturning, so we see that saturation breaks down in our idealized model at 'realistic' 


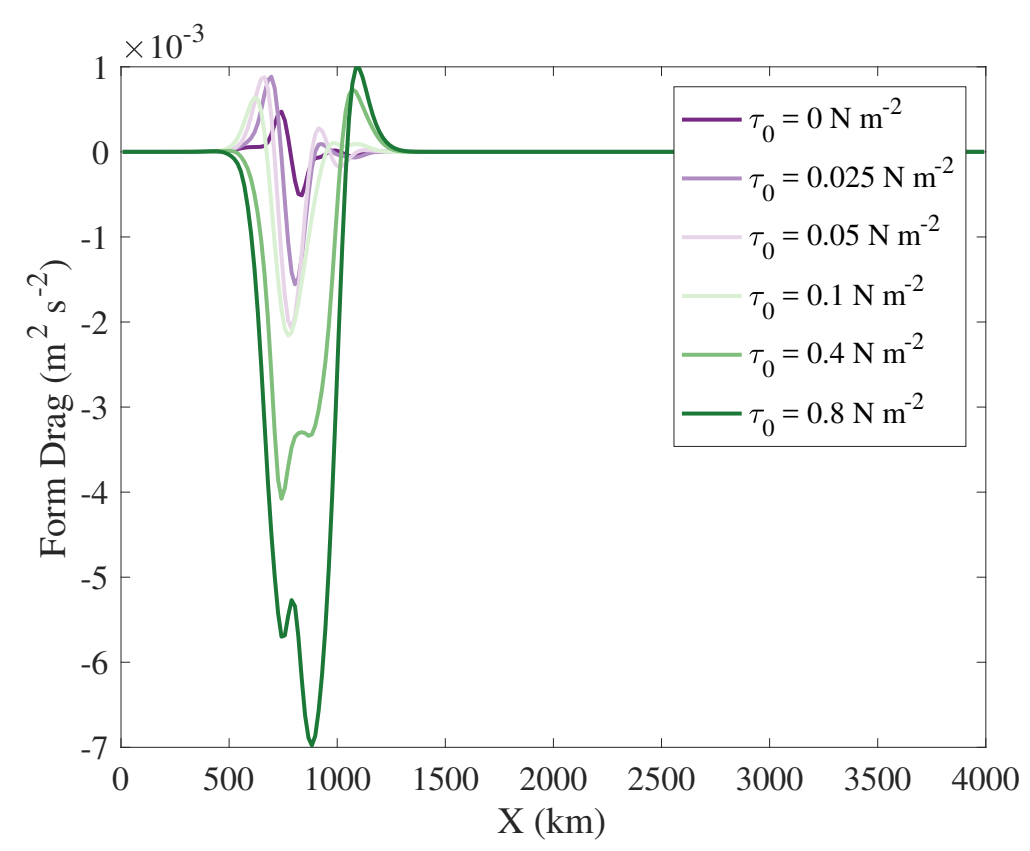

Figure 3-6: Form drag along the channel for $\mathcal{H}_{0}=-2 \mathrm{~Sv}$. As the wind forcing decreases, the form drag becomes both weaker and more symmetric, but doesn't disappear altogether, leading to smaller net heat fluxes in the meander (Fig. 3-5).

wind stresses.

So what is happening as the wind forcing gets weaker? The dominant momentum balance is given by Equation 3.11 , or, to lowest order,

$$
\frac{\tau}{\rho_{0}}=f_{0}\left\langle h_{b} \bar{\psi}_{2 x}^{\prime}\right\rangle
$$

while $\tau_{0}>0.0125 \mathrm{~N} \mathrm{~m}^{-2}$. As the wind forcing weakens, the form stress also weakens, which can happen either by reducing the magnitude of $\bar{\psi}_{2 x}^{\prime}$, the lower layer time averaged stream function, or by reducing its correlation with $h_{b}$. In these simulations both of these mechanisms occur. At zero wind, the form stress $h_{b} \bar{\psi}_{2 x}^{\prime}$ is not zero everywhere, but it is symmetric about the ridge (Fig. 3-6). Because of the symmetry, the form stress on one side of the ridge cancels out the form stress on the other side of the ridge, leading to no net form stress.

In order to relate the changes in form stress to the isopycnal slopes we must consider the connection between the form stress and the total eddy heat fluxes. The total eddy heat fluxes are given by $F_{1}\left\langle\overline{\psi_{1}^{\prime} \psi_{2 x}^{\prime}}\right\rangle$ which we can split up to be $F_{1}\left\langle\overline{\psi_{1}^{\prime} \psi_{2 x}^{\prime}}\right\rangle=F_{1}\left\langle\overline{\psi_{1}} \overline{\psi_{2 x}}\right\rangle+$ transients, where the first term on the right hand side, the standing eddy contribution, dominates over 


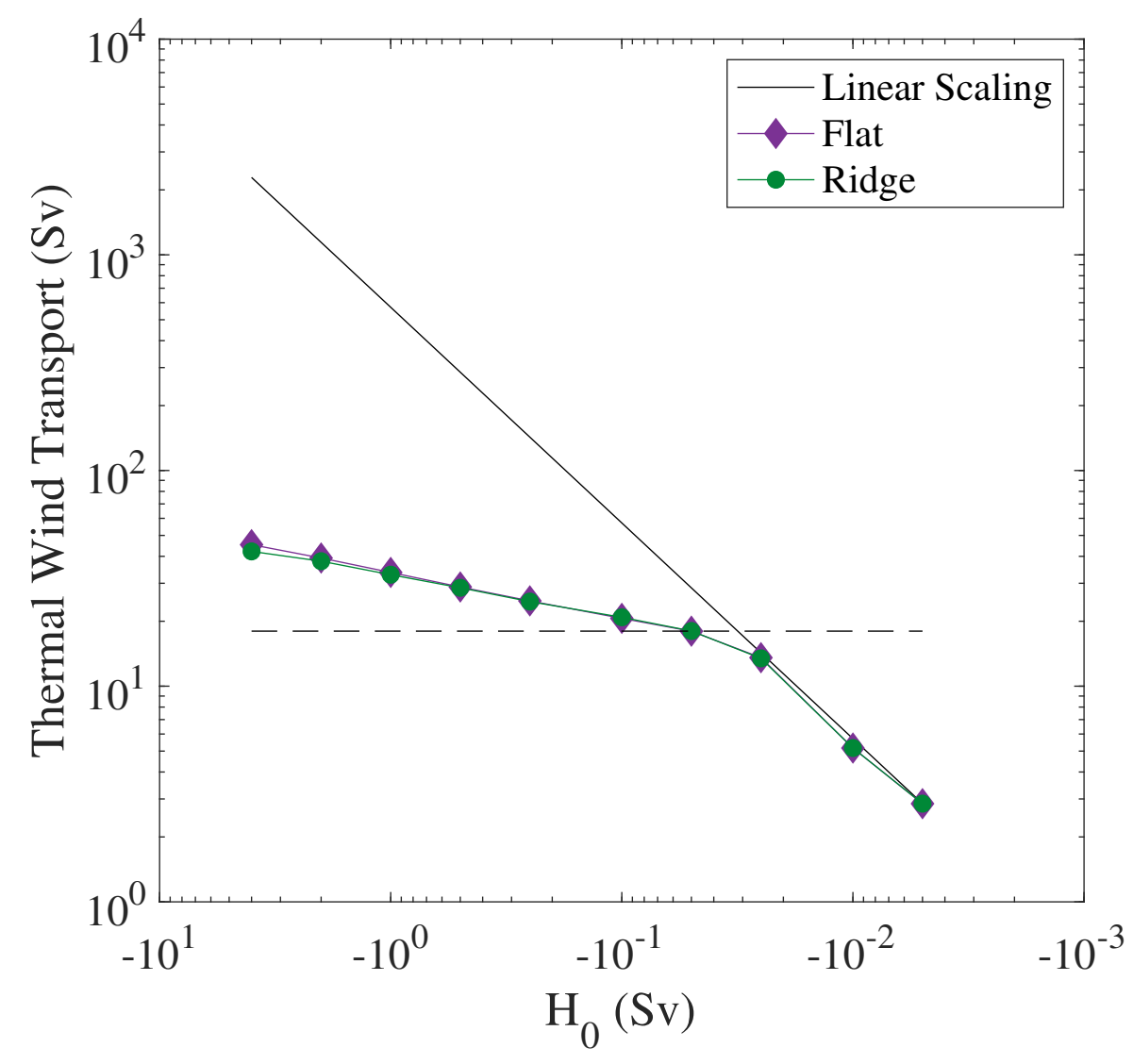

Figure 3-7: Thermal wind transport versus residual overturning forced along interface for entire $\mathcal{H}_{0}<0$ range. The linear scaling developed is plotted against the simulated results that works well until the flow becomes eddying. The dashed line shows where the flow reaches its critical shear for instability following the Phillips problem. The thermal wind transport scales directly with the residual overturning and provides the no-wind limit to the thermal wind transport.

the transient term. So, if the magnitude of $\bar{\psi}_{2 x}^{\prime}$ decreases then the eddy heat fluxes in the meander will decrease. The correlation between these two terms could also decrease 6 . In general, we see that $\bar{\psi}_{1}^{\prime}$ stays relatively symmetric about the ridge because the upper layer only sees the ridge through the lower layer (Fig. 3-3). As $\bar{\psi}_{2}^{\prime}$ becomes more symmetric about the ridge, the correlation between $\bar{\psi}_{1}^{\prime}$ and $\bar{\psi}_{2 x}^{\prime}$ will become smaller in the mean because the stream functions become more similar, leading to a cancellation of heat fluxes on either side of the ridge (Fig. 3-5(b)). As the wind forcing decreases, the meander becomes smaller and more symmetric about the ridge and the ratio of the contribution to the fluxes from the meander and the rest of the domain decreases.

\footnotetext{
${ }^{6}$ The correlation here could also decrease because the flow barotropizes and $\psi_{1}$ and $\psi_{2}$ become more similar reducing the similarity between $\psi_{1}$ and $\psi_{2 x}$.
} 


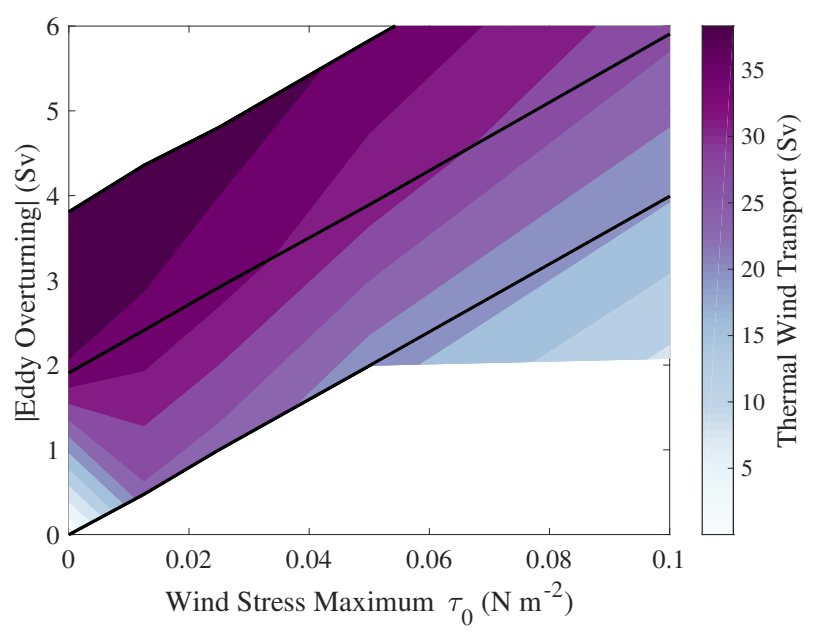

Figure 3-8: A figure showing the thermal wind transport versus the wind stress and total eddy overturning. The black lines correspond to a constant residual overturning. We see that for a given wind forcing, a stronger eddy overturning corresponds to a stronger thermal wind transport and for a given eddy overturning, a stronger wind forcing corresponds to a weaker thermal wind transport. However, as we increase the wind forcing along a line of constant overturning, the decreasing effect of the wind forcing overpowers the increasing effect of the eddy overturning, leading to smaller thermal wind transports as we increase the wind forcing for a negative residual overturning.

The result of the standing meander playing a smaller role in the heat fluxes is an increased sensitivity to wind forcing, but why do the thermal wind transports fan out as we observe (Fig. 3-4)? With strong wind stress, the cumulative eddy fluxes are concentrated in the meander, but as the wind forcing weakens the fluxes become important throughout the domain until the heat fluxes match the values for a flat-bottomed configuration (Fig. 35(b)). In this new regime, where the fluxes depend on the mean flow structure over the whole domain, the dynamics approach those of the flat-bottomed simulations where the eddy fluxes are uniform in $x$; thus the thermal wind transport limits to the flat-bottomed transport as the winds weaken.

\subsubsection{No-wind Limit}

Since the ridge simulations approach the flat-bottomed ones as the wind forcing weakens, it is important to understand the flat-bottomed simulations with no wind forcing. Intuitively, we would expect that the isopycnal slope change would be in proportion to the magnitude of the changes in the heat flux. We illustrate this using a linear approximation to our system, with no wind forcing and no eddies. The buoyancy forcing contribution to the upper layer 
zonal mean potential vorticity equation can only be balanced by the $\kappa$ term, the potential vorticity diffusivity, in the steady state i.e.,

$$
0=-\frac{f_{0} \mathcal{H}}{H_{1}}+\kappa \nabla^{2}\left\langle q_{1}\right\rangle
$$

Assuming that the potential vorticity is dominated by the stretching term, or $\left\langle q_{i}\right\rangle \approx$ $\mp F_{i}\left(\left\langle\psi_{1}\right\rangle-\left\langle\psi_{2}\right\rangle\right)$, which is a good assumption considering the size of the domain, yields the upper-layer balance:

$$
\frac{f_{0} \kappa}{g^{\prime}} \nabla^{2}\left(\left\langle\psi_{1}\right\rangle-\left\langle\psi_{2}\right\rangle\right)=\mathcal{H}_{0} \sin (\pi y / L)
$$

We define $\psi_{B C}=\left\langle\psi_{1}\right\rangle-\left\langle\psi_{2}\right\rangle$ and assume a solution of the form $\psi_{B C}=\hat{\psi}_{B C} \sin (\pi y / L)$. We plug this into Equation 3.13 and find that $\hat{\psi}_{B C}=g^{\prime} \mathcal{H}_{0} L^{2} /\left(f_{0} \kappa \pi^{2}\right)$, which tells us that the thermal wind transport, $\left\langle\overline{u_{1}}\right\rangle-\left\langle\overline{u_{2}}\right\rangle$, scales with $\mathcal{H}_{0}$, the magnitude of the heat fluxes. We compare this to the fully-nonlinear channel model with and without topography in Fig. 3-7. With no wind stress and only heat flux, we see that the thermal wind transport follows our linear scaling until the overturning reaches $\mathcal{H}_{0}=-0.05 \mathrm{~Sv}$, at which point, eddies become important and the transport scales approximately like $\mathcal{H}_{0}^{1 / 5}$ (Fig. 3-7 7 . It is clear however, as the forcing continues to grow, the thermal wind transport continues to grow with it, indicating that stronger heat fluxes do indeed generate steeper isopycnal slopes, a pattern that holds even with wind forcing and an eddying flow. Since the thermal wind transport must approach the values determined by the residual overturning when there is no wind forcing, the thermal wind transport can not be saturated for every wind forcing in order to satisfy this limit.

\subsubsection{Connecting the Limits}

Our results lead to the question of why must it be the case that the thermal wind transport decreases with increasing winds with topography but strengthens in the flat-bottomed case for a negative overturning. This pattern of decreasing thermal wind transport with an increase in wind is also seen, but not rationalized, in the channel models of Mak et al. 2018 and Munday and Zhai 2015. The thermal wind transport depends on the total

\footnotetext{
${ }^{7}$ It is an open question why the $1 / 5$ th dependence exists in particular, however the weaker dependence on $H_{0}$ is expected due to eddies acting to flatten the isopycnal slope and reduce the sensitivity to the forcing.
} 
eddy overturning and the wind forcing, but these two components vary with wind forcing differently. With stronger wind forcing, there is a smaller thermal wind transport because the meander grows and becomes asymmetric, isolating fluxes and increasing the eddy efficiency Abernathey and Cessi, 2014, leading to flatter isopycnal slopes (Fig. 3-8). There is a singularity where the wind stress is zero because the total eddy overturning also goes to zero. With a stronger eddy overturning, there is a larger thermal wind transport because generally steeper isopycnal slopes lead to larger eddy fluxes and eddy overturning (Fig. 3-8). If we consider simulations where the residual overturning is constant, then when we increase the wind forcing, the eddy overturning increases as well, leading to opposing steepening and flattening of the isopycnals. We see that, of these two effects, the increase in eddy efficiency due to the meander wins over the need for more eddy activity (Fig. 3-8. Thus, the thermal wind transport decreases with an increasing wind forcing when there is a negative overturning.

\subsection{Discussion and Conclusions}

The thermal wind transport in the idealized Southern Ocean-like channel model employed here is sensitive to changes in wind at 'realistic' wind stresses, between 0.1 and $0.2 \mathrm{~N} \mathrm{~m}^{-2}$, albeit in this idealized framework it is hard to make quantitative comparisons. At wind stresses below $\tau_{0}=0.4 \mathrm{~N} \mathrm{~m}^{-2}$, the transport either increases or decreases with the wind depending on the residual overturning because the simulations limits to those with a flat bottom as the winds weaken (Fig. 3-9). The convergence happens because for weaker wind stress the amplitude of the standing meander reduces and thus the system becomes more zonally symmetric as in a flat bottom channel. Our results and arguments show the limitations of eddy saturation as a theory even in an idealized configuration when there is a residual overturning.

Our results differ from previous studies, in part because we isolate the positive and negative overturning cells. This is different than Hogg 2010 where both negative and positive buoyancy fluxes happen at once. Hogg 2010 also lacks a proper residual overturning where there is a meridional circulation at depth, but instead has all the meridional transport occurring in a mixed layer, and the wind forcing is also happening in different location as the buoyancy forcing. Hallberg and Gnanadesikan 2001 also only considers a positive 


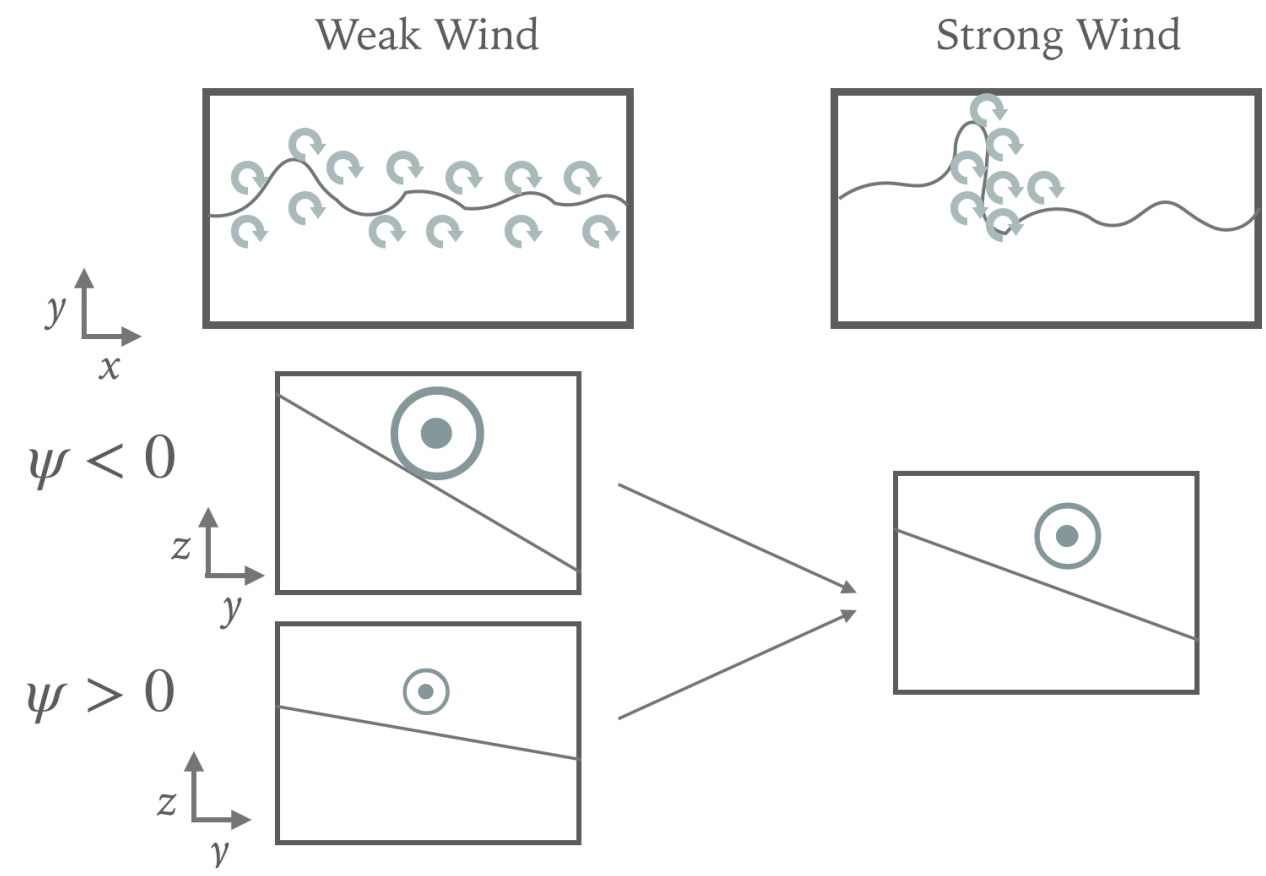

Figure 3-9: A concluding schematic. When there is a weak wind forcing, the strength of the thermal wind transport is dependent on the sign of the overturning, the meander is symmetric about the ridge, and the eddy activity is spread throughout the domain. When the wind forcing is strong the eddy activity is localized to the meander region, the meander is asymmetric, and the thermal wind transport no longer depends on the overturning.

overturning, so they cannot see the different behavior with the negative overturning. It is possible that our results would be consistent with these past studies if we considered similar forcing regimes and could look at each overturning cell individually.

This study does not directly address why eddy saturation exists in these simplified models, but hopefully, by extending the work of Abernathey and Cessi 2014 and Nadeau and Ferrari 2015, it provides insight into the relative importance of the meander. Working to understand the meander spin-up process also merits further study. It is not clear why the standing meander is so much more efficient at flattening isopycnal slopes than the winddriven process of increasing the slopes. Mak et al. [2018] encapsulates the eddy efficiency in their $\alpha$ parameter. In our configuration this $\alpha$ must change if the transport changes; there are no other parameters that could vary to change the transport, though it is unclear how or why the eddy efficiency changes.

One other open question is what sets the value of wind stress where saturation begins. The saturation does not depend on the relative strength of the wind-driven overturning, 
but the absolute wind stress. It seems likely to depend on when the isolation of the fluxes to the ridge area begins. Understanding the isolation process has serious implications for predicting how the Southern Ocean thermal wind transport and residual overturning will respond to changes in the wind.

The geometry is simpler than the real Southern Ocean, but the dominant feature of a blocking ridge is present. The ridge is too small compared to real topographic features Thompson and Naveira Garabato, 2014, so the simulations have been repeated with a $2000 \mathrm{~m}$ tall ridge, and the saturated thermal wind transport value is the same. The main difference is that the saturation breaks down closer to $\tau_{0}=0.3 \mathrm{~N} \mathrm{~m}^{-2}$ versus $\tau_{0}=0.4 \mathrm{~N} \mathrm{~m}^{-2}$ for a $1000 \mathrm{~m}$ tall ridge, but the main results still hold. Another potential limit to our result is the use of two-layer quasi-geostrophic simulations. The simplified dynamics could lead to potential problems due to the lack of outcrops as seen in the real Southern Ocean, so to this end, we will be carrying out analogous investigations in primitive models using MITgcm simulations with the same geometry; preliminary experiments are broadly consistent with the results presented in this study. Another approach to address this problem would be to consider a three-layer quasi-geostrophic model to extend our result with both a positive and a negative cell at the same time. It is unclear how these results would translate into primitive equation channel models, especially whether eddy saturation would still kick in only at unrealistically strong wind stresses.

Our system is simple in order to fully explore the parameter space of baroclinic instability. The result from this study is useful for considering more complicated models and provides a framework to analyze changes in thermal wind transport with wind based on the magnitude of the residual overturning. We show that both topography and residual overturning are vital components for any theory of saturation. It is possible that saturation seen in various models and the real Antarctic Circumpolar Current with two overturning cells is actually due to the wind forcing changing the thermal wind transport in opposite directions in each overturning cell. If we connect these isopycnal slope patterns to outcrop positions in the real ocean, then we could also provide insight into the changes of the residual overturning with wind and the implications for fluxes of heat and other important properties such as carbon. 


\section{Chapter 4}

\section{Causes of Localized Upwelling in a Southern Ocean-like Channel Near Topography}

\subsection{Introduction}

The Southern Ocean is key to our understanding of climate change because it plays an outsized role in the global carbon budget. How that carbon budget has varied since the industrial revolution and how the budget will change with climate change are open questions. Since there is a strong vertical gradient in dissolved inorganic carbon in the near-surface Southern Ocean, the vertical transport of water is a major physical process affecting the carbon fluxes. Thus an understanding of upwelling is necessary to understand the carbon budget.

Physically, we call the circulation responsible for the upwelling of tracers the residual overturning circulation. This is called the residual circulation because it is the small remainder of two main competing processes, wind-driven overturning that steepens isopycnals and eddy-driven overturning that acts to relax those isopycnals Marshall and Speer, 2012. This understanding was developed for an idealized ocean that lacks bottom topography or other zonal asymmetries. Many other aspects of this theory have been shown to be wrong due to the existence of topography Youngs et al., 2017, 2019, Abernathey et al., 2011. This leads us to question how the topography contributes to the upwelling of tracers. 
One aspect that is just recently starting to be understood is that upwelling seems to be localized to regions downstream of topography [Tamsitt et al., 2017]. More specifically, we know that overturning is isolated from modeling studies showing localized upwelling of particles near topographic features [Tamsitt et al., 2017]. The localized upwelling has been suggested to be due to baroclinic eddies enhanced downstream of topography. However, there is a gap in understanding and evidence for specific dynamical processes that drive the overturning. In this paper, we will address this gap by showing specifically which processes are leading to the vertical transport of water in which locations.

In this study, we use an MITgcm channel model representing the Antarctic Circumpolar Current, with idealized geometry and forcings, to understand the driving forces of the overturning. We build off a two-layered simulation with similar parameters in Chapter 3 and with a similar framework Youngs et al., 2017] in Appendix B. In this paper we find that eddies are localized near topography and so is the eddy-driven upwelling. In addition the mean flow around the topography plays a major role in setting the overturning. This implies that we need to investigate very near to topography to understanding the vertical fluxes of tracers.

In this paper, we describe the numerical model in section 2. In section 3, we expand on the particle tracking method used. In section 4 we present our results, and in section 5 we discuss and conclude.

\subsection{Numerical Model}

We use an MITgcm channel that is $4000 \mathrm{~km}$ long by $2000 \mathrm{~km}$ wide and re-entrant in longitude (Figure 4-2). The model is run at a $10 \mathrm{~km}$ resolution. The deformation radius in this configuration is about $15 \mathrm{~km}$, but features tend to be larger than the deformation radius Pedlosky, 1987. As a check, we also ran the simulations at a $5 \mathrm{~km}$ resolution and saw the same physical behavior. We have a total depth of $4000 \mathrm{~m}$ with 32 points in vertical, from 10 $\mathrm{m}$ grid spacing at the surface to 280 meters in grid spacing at the bottom. For topography we have a Gaussian ridge of half-width $200 \mathrm{~km}, 2000 \mathrm{~m}$ tall, located at $800 \mathrm{~km}$ downstream of the channel entrance (Figure 4-2). We use a 600 second time step, free slip sides, and a diffusivity varying from $0.01 \mathrm{~m}^{2} \mathrm{~s}^{-1}$ to $1 \times 10^{-5} \mathrm{~m}^{2} \mathrm{~s}^{-1}$ exponentially. We also have a linear bottom drag with a drag coefficient of $1.1 \times 10^{-3} \mathrm{~m} \mathrm{~s}^{-1}$. 


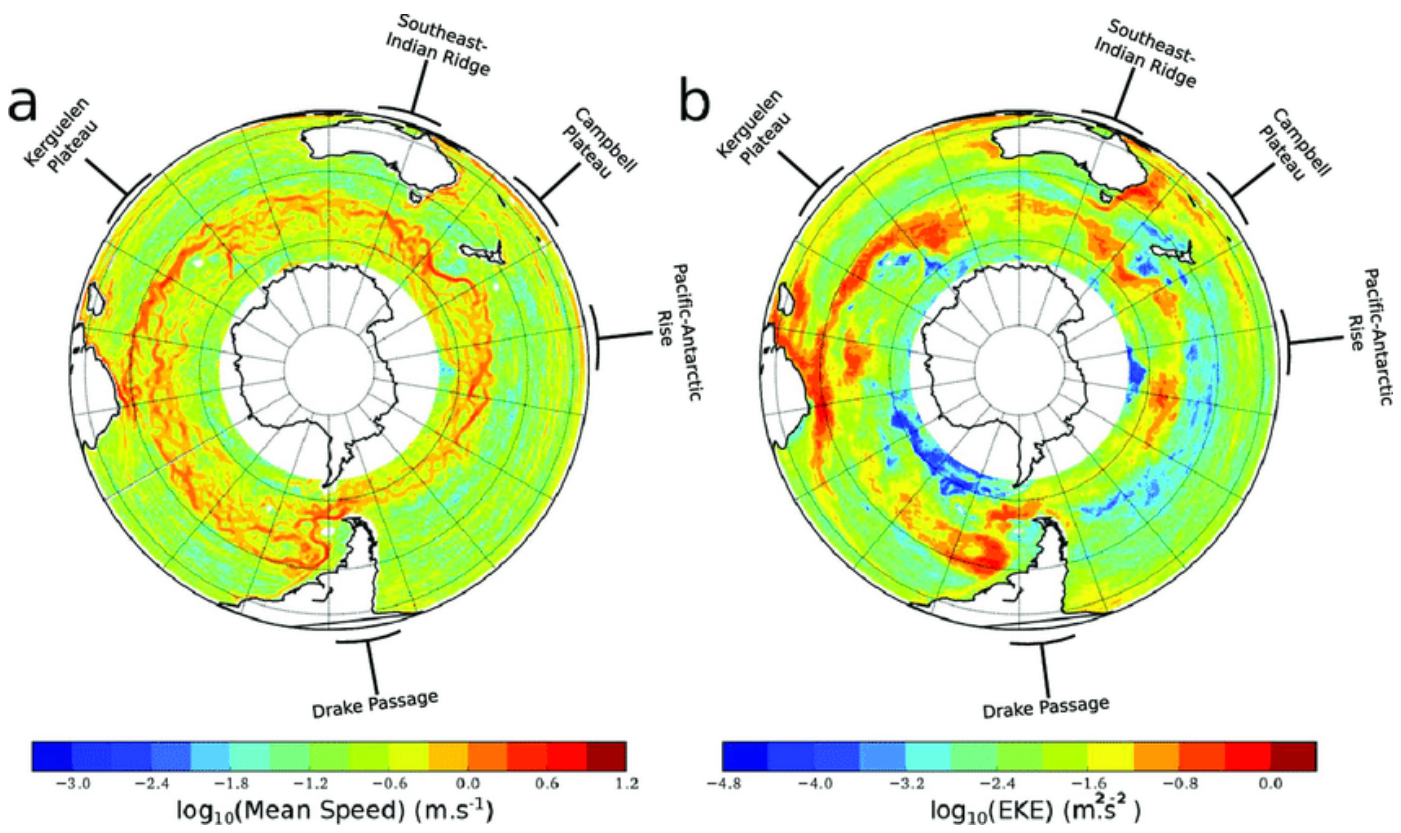

Figure 4-1: Although the Southern Ocean is often thought about in a zonally symmetric manner, the Southern Ocean indeed has locations where properties, such as surface velocity as shown here, deviate highly from the zonal average. Figure from Chapman et al. 2015.

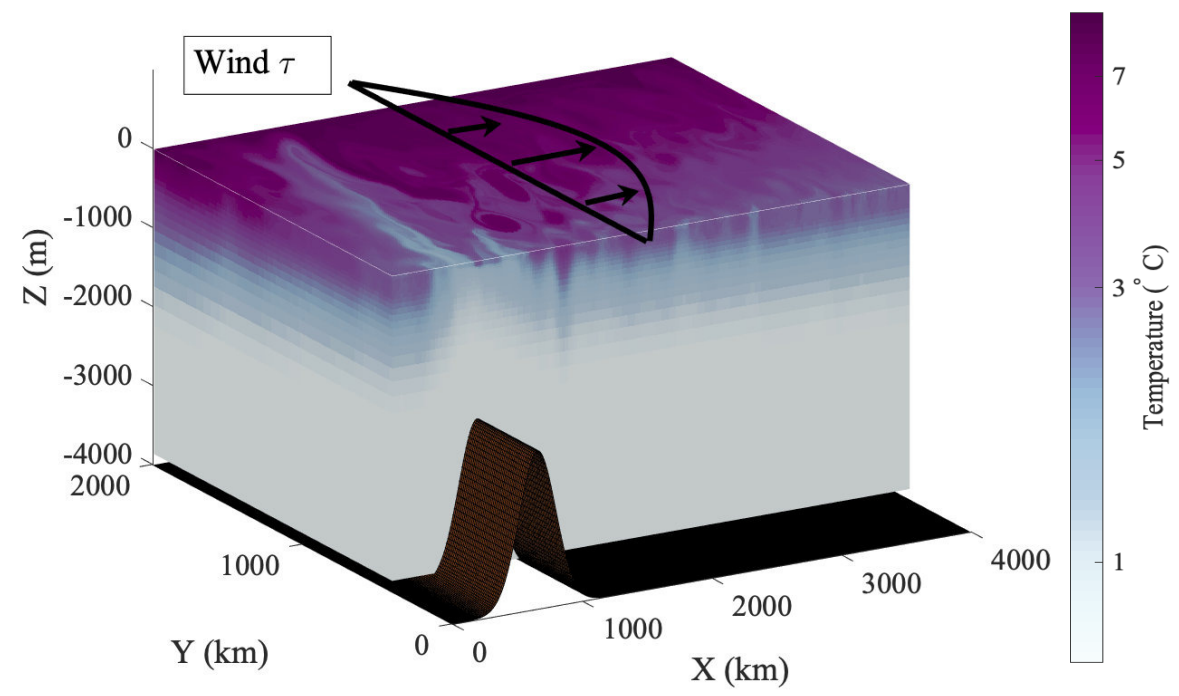

Figure 4-2: Geometry of the idealized channel. Ridge at $800 \mathrm{~km}$, wind has a sine profile with a maximum $\tau_{0}$, the color here is snapshot temperature with $\tau_{0}=0.15 \mathrm{~N} \mathrm{~m}^{-2}$. 


\subsubsection{Boundary Conditions}

The surface is forced using a wind stress of the form

$$
\tau(y)=\tau_{0} \sin \left(\pi y / L_{y}\right)
$$

where $L_{y}=2000 \mathrm{~km}$. For the base case, $\tau_{0}=0.15 \mathrm{~N} / \mathrm{m}^{2}$; it will be varied from 0.05 to 0.25 $\mathrm{N} / \mathrm{m}^{2}$ (Figure $4-2$ ).

At the northern boundary, the temperature (which determines the buoyancy) relaxes to a fixed profile with a timescale of 1 week, as an exponential profile

$$
T_{n}(z)=\Delta T\left(e^{z / h}-e^{-H / h}\right) /\left(1-e^{-H / h}\right)
$$

where $T_{n}$ is the temperature at the northern boundary, $H$ is the domain depth of 4000 $\mathrm{m}$, and $-h$ is a decay depth of $1000 \mathrm{~m}$. The choice of profile is validated by Karsten and Marshall 2002.

We use a surface relaxation condition because it allows the strength of the residual to change as we change the wind. We use the following method because a fixed surface relaxation temperature profile for both the flat bottom and ridge cases leads to wildly different overturnings so our method allows both a relaxation boundary condition and comparable overturnings for the flat bottom and ridge simulations. We first run simulations with a fixed flux surface boundary condition as shown in Figure 4-3(b). From this simulation, we use the time- and zonally-averaged surface temperature to choose a relaxation temperature profile that recreates the fixed flux profile following the equation $Q=-\lambda\left(\langle T\rangle-T_{0}\right)$ where $Q$ is the heat flux, $\lambda$ is the relaxation time scale of one month and $T_{0}$ is the temperature profile calculated. This calculation is done both for the flat-bottomed and ridge cases. This method of using the fixed flux conditions in order to generate a temperature relaxation is required because the isopycnal slopes are very different between these two cases. The heat fluxes are not zonally symmetric and are visibly enhanced near topography due to colder water being brought northward (Figure 4-3(d)). 

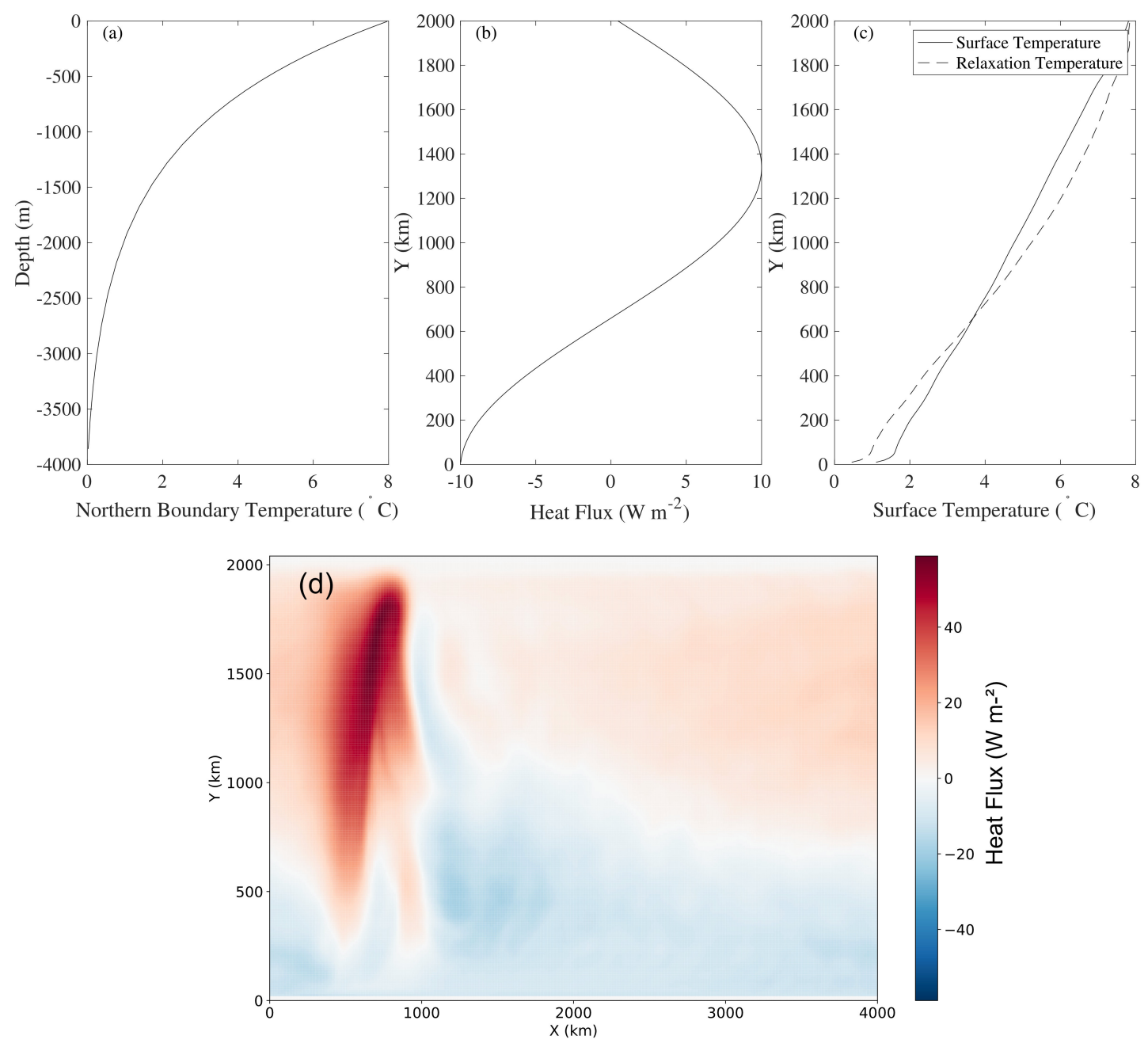

Figure 4-3: Figure showing the temperature boundary conditions. (a) Northern boundary relaxation temperature. The temperature is relaxed linearly from the maximum at the northern boundary, linearly scaling back to no forcing at $1930 \mathrm{~km}$. (b) Heat flux profile used with the average surface temperature (c) to generate surface relaxation temperature. (d) shows the full heat flux pattern for $\tau_{0}=0.15 \mathrm{~N} \mathrm{~m}^{-2}$. 


\subsubsection{Results}

We then can calculate the residual overturning in the traditional way:

$$
\psi=\int \overline{v h} d T
$$

where $h$ is the temperature (buoyancy) layer thickness, $v$ is the meridional velocity in the layer. We show the meridional overturning circulation for both the flat bottom and ridge cases (Figure 4-4). The simulations with a ridge show an extra feature in the overturning due to the surface heat fluxes associated with the meandering flow. When this overturning is then projected into depth-space, the surface meander feature is projected below the surface ocean, so the zonal average is missing important aspects of the residual circulation. One difference between the flat bottom and the ridge is the steepness of the isopycnal slopes. The isopycnal slopes are much steeper for the flat-bottomed simulations, leading the lower cell to be much deeper in the domain than with the ridge simulations. This has implications for which water masses reach the surface and where they are exchanged with the rest of the ocean.

We calculate the overturning strength versus the wind stress maximum for the ridge simulation. The upper cell increases less than linearly with the wind due to compensation of the meander, in agreement with previous studies Munday et al., 2013, Morrison et al., 2011]. The lower cell does not change much at all. On the other hand, the meander cell does get stronger, indicating that the flow becomes faster and sharper around the topography. This cell is a feature due to the meander where water at the surface warms as it travels northward in the meander and cools as it travels southward, appearing as an overturning cell but is actually just flow around the topography. This 'meander cell' is the same feature that is mistakenly projected to depth in Figure 4-4. For the remainder of this paper, we will focus on the upper and lower cells for the meander cell doesn't involve vertical transport, just horizontal transport.

\subsection{Particle Tracking}

We want to answer where and why upwelling happens in the Southern Ocean. In order to understand the Lagrangian and residual circulation of the Southern Ocean, we employ 

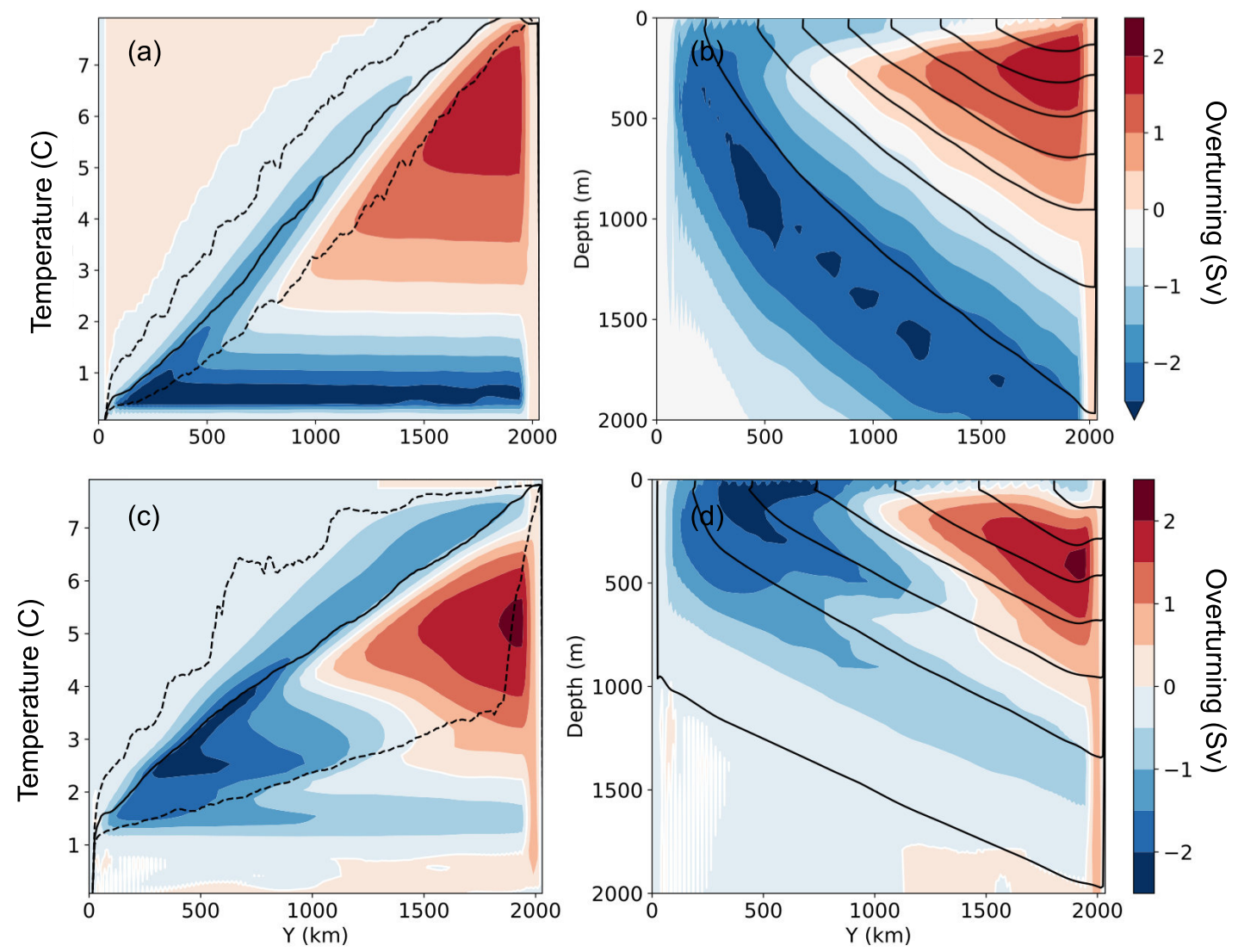

Figure 4-4: Residual overturning in temperature space (a,c). The same overturning projected into depth coordinates $(b, d)$. The overturning is shown for flat-bottomed simulations $(a, b)$ and ridged simulation $(\mathrm{c}, \mathrm{d})$.

particle tracking. We use the python package OceanParcels (oceanparcels.org). We advect the particles in 1 day averages of the flow. We set up a $100 \mathrm{~km}$ by $100 \mathrm{~km}$ grid and release particles in the center of the box for every $\mathrm{z}$ on the native grid. Then we advect the particles until they leave the box. We then tag the particle with the amount it has upwelled to its starting point and average all the particles in the box. This produces a map of how much a particle has upwelled in each grid box due to total flow. In addition, We have advected particles vertically with different components of the vertical velocity only. We split the vertical velocity into mean and eddy and then split the eddy into baroclinic and barotropic eddies, where the barotropic component is the depth averaged flow and the baroclinic is the difference between the total and the barotropic. We release new particles every 10 days and run for 360 days which we call one year. 


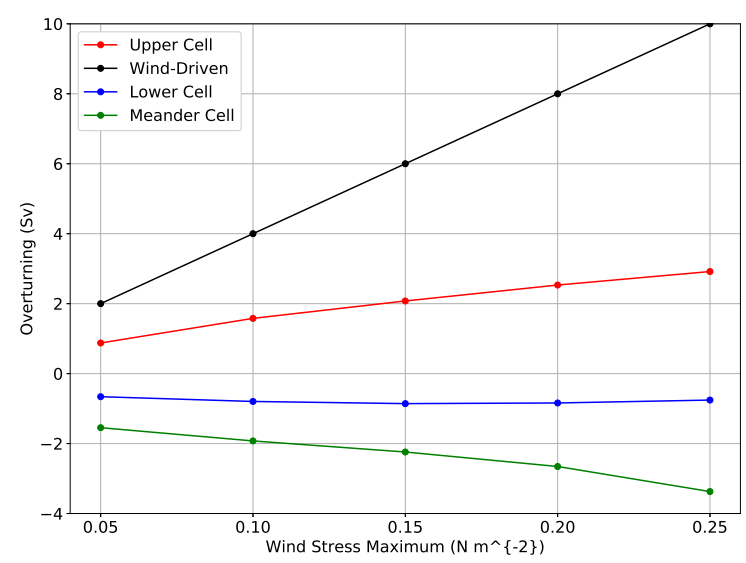

Figure 4-5: Overturning strength versus wind stress maximum for the ridge simulation. The upper cell is the maximum overturning, the lower cell is the minimum at the northern boundary and meander cell is the minimum throughout the domain.

\subsubsection{Alternate Particle Tracking Method}

In addition to tracking particles until they leave their grid boxes, we have run experiments calculating the upwelling of particles in one circuit around the channel. We release particles $\mathrm{x}=100 \mathrm{~km}$ and every $\mathrm{y}$ and $\mathrm{z}$ location on the native grid, near the entrance to the channel. We throw out particles that enter mixed layer, and those that enter the northern temperature relaxation region. We analyze the particles based on the initial temperature and throw out particles if they change temperature too much $( \pm 0.1 \mathrm{C})$. We use the main method in addition to this one because the sampling using this method is biased, due to only sampling where the particles travel. However, these experiments are useful to include for their relatively better representation of the flat-bottomed experiments. We show the vertical displacement following these particles in figure 4-7. We see that the particles go up and down over the topography and then generally see enhanced upwelling downstream of the topography and then a leveling out far downstream due to steepening isopycnals.

\subsubsection{Validation}

In this section, we seek to validate the particle tracking method for our use as a tool to understand the upwelling in the Southern Ocean. Ideally, we want our method to be robust to small parameter changes and not biased in sampling to create an accurate representation 


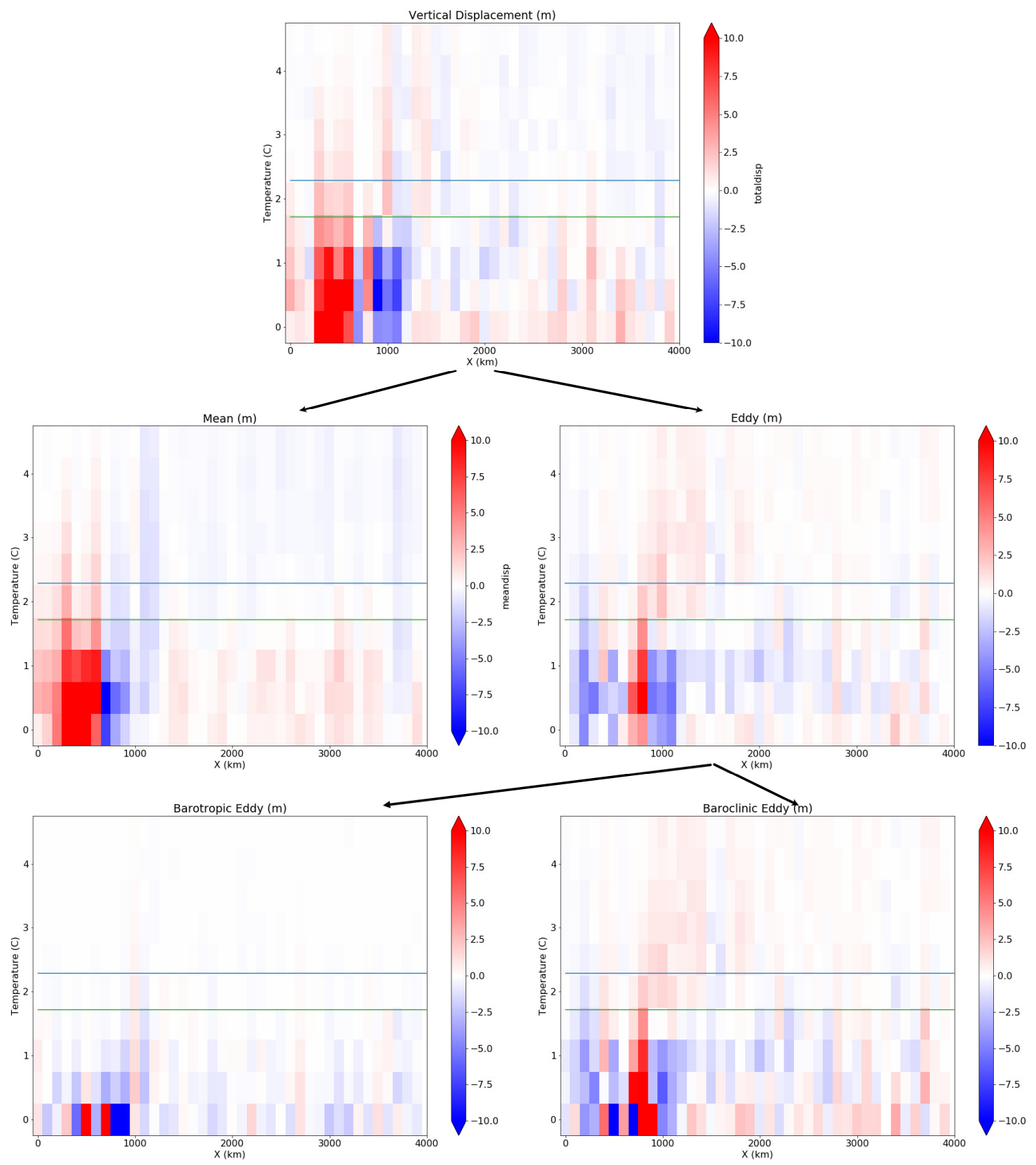

Figure 4-6: The vertical displacement of particles as they exit their initial boxes. We split the vertical displacement into mean and eddy and then further to the barotropic and baroclinic eddies. The blue line separates the upper and lower cells, the green line separates the upwelling and downwelling in the lower cell. The time required for particles to leave each box varies widely depending on the horizontal velocities with order 10 days on average.

of the Southern Ocean overturning. We validate the method here by comparing the vertical velocities calculated from the particles and those calculated by MITgcm (Figure 4-8). Since 


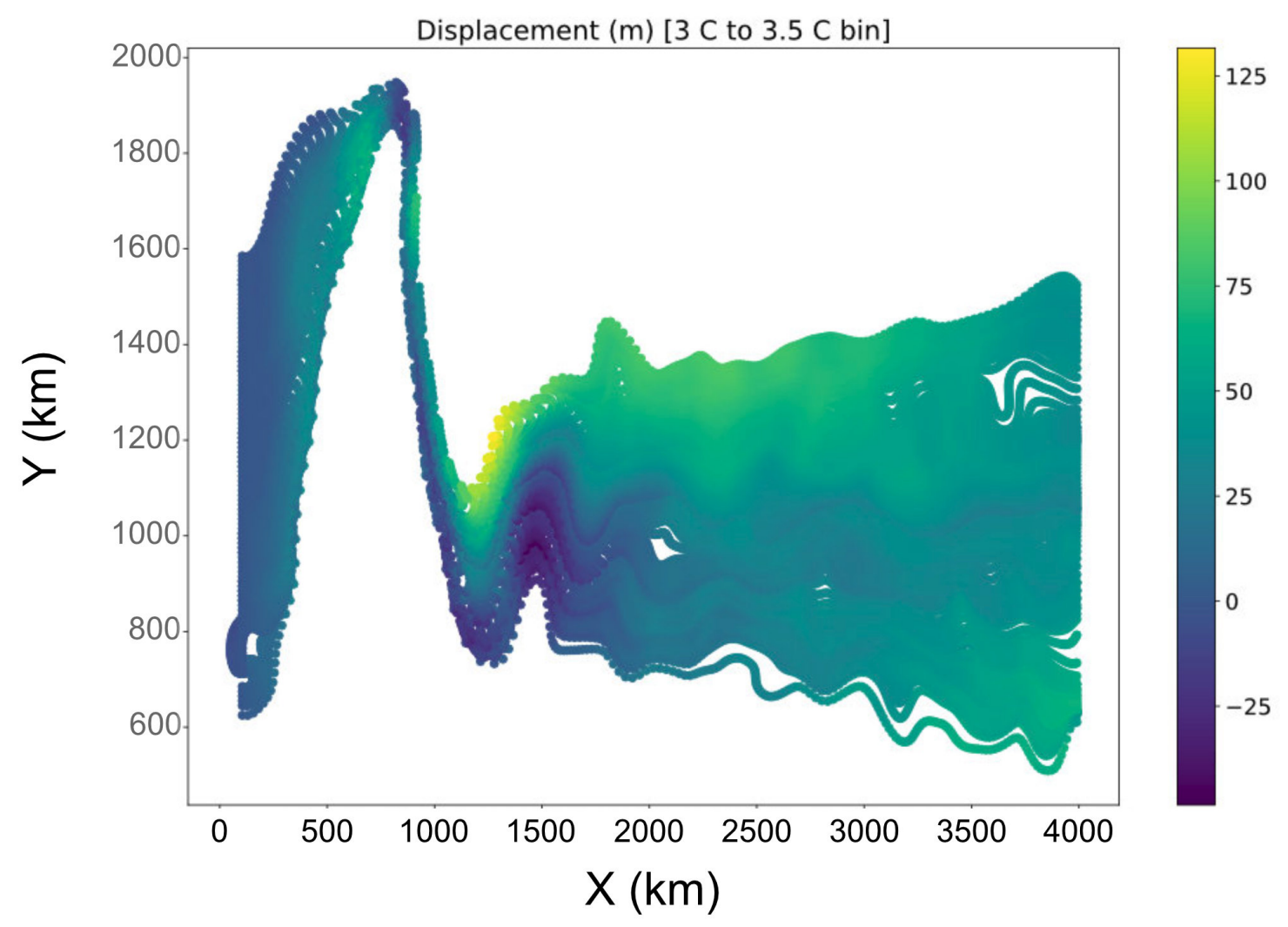

Figure 4-7: Vertical displacement along particle trajectories from the alternate particle tracking method showing how the the displacement behaves over a long time period.

the mean velocity calculated by the particles is similar enough to the mean velocity calculated by the model, we believe that each of the following parameters is sufficiently high enough frequency or long enough time averaged.

\section{Sensitivity to output frequency}

We advect particles in 1-day, 5-day, and 10-day averaged flows starting from an x,y,z grid of every $100 \mathrm{~km}$ in $\mathrm{x}$ and $\mathrm{y}$ and every point in the native $\mathrm{z}$ grid. The initial conditions are identical for each round of particle advection. To analyze, we look at histograms of temperature drift for each of these runs, as well as histograms of the divergence of the particles from the 1 day averages.

Between the 5 and 10 day runs, the temperature drift distribution is broader for the 10 day run, indicating that with the lower-frequency periods, the particles are leaving their initial temperature surfaces more, suggesting that the higher frequency data is necessary. 


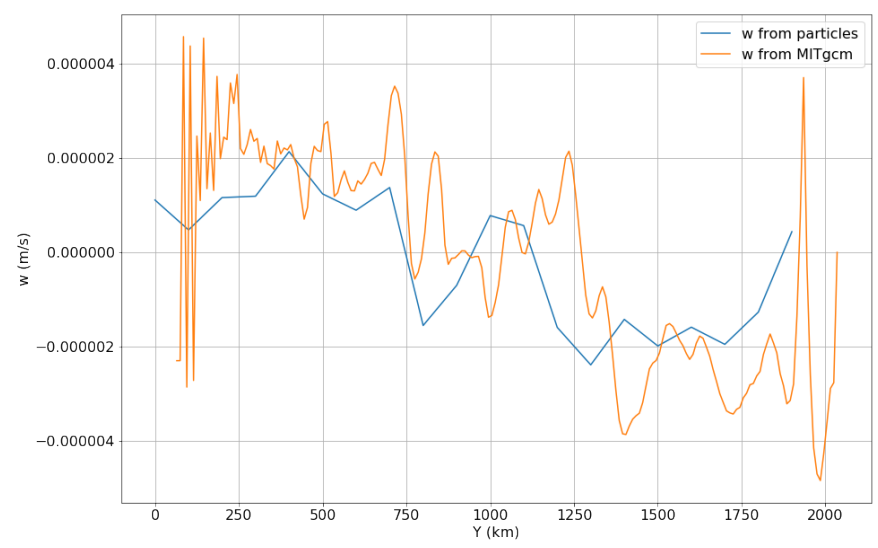

Figure 4-8: A figure showing the mean vertical velocities calculated from particles and from MITgcm output over a 5 day period. They agree to lowest order, however they are not identical as w calculated on an isopycnal not identical to the Eulerian w calculated in the model.
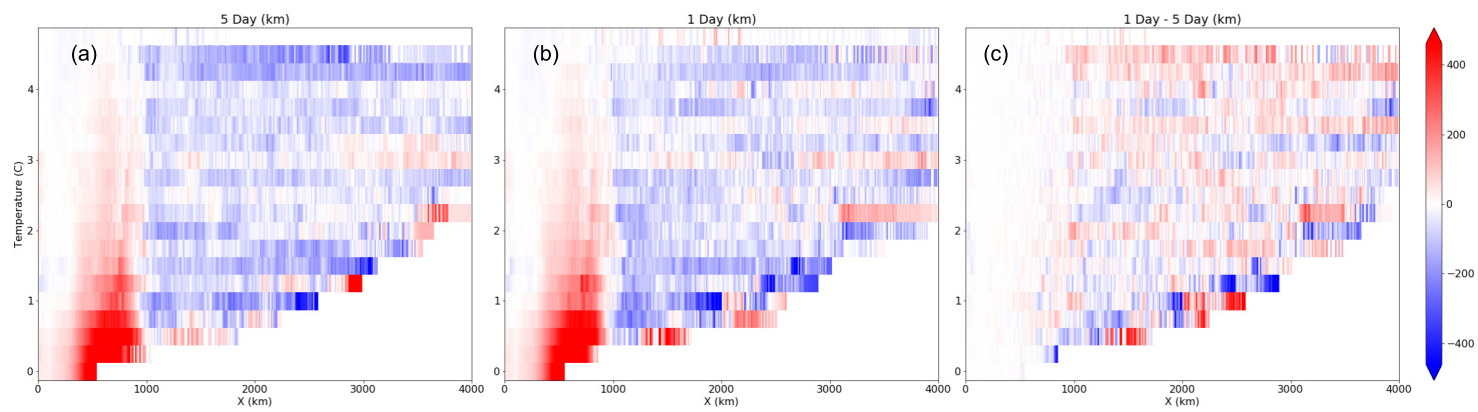

Figure 4-9: Particle tracking of particles released at the channel entrance in both 5 day averaged and 1 day averaged velocities. The difference shows more upwelling for the 1 day average, indicating that it is necessary to appropriately represent upwelling.

The temperature distribution is similar for the 5 day and 1 day output, indicating that the higher frequency output represents the residual (or flow along isopycnals) more accurately.

The histograms of the divergence of the particles show that over 60 days, the particles generally stayed within $10 \mathrm{~km}$ of the reference particles, whether the reference was the 1 day particles or the 5 day particles. Generally the convergence was better for the 5 day particles than the 10 day particles, however the distribution for the 10 day particles doesn't seem that bad. We are happy with the convergence here. Within $10 \mathrm{~km}$ is pretty close for at 2000 $\mathrm{km}$ by $4000 \mathrm{~km}$ channel over 60 days. For reference $10 \mathrm{~km}$ is one grid point. In addition, we used the alternate method of tracking particles released at the channel entrance and found more upwelling in the 1 day averaged flow, indicating that 1 day averages are necessary to accurately represent upwelling (Figure 4-9) 
Because of all of these metrics we use 1 day upwelling. We don't expect 12 hours to be necessary because mesoscales have a timescale of days, although it maybe more relevant to consider the timescale required to circulate the eddy rather than the timescale of the eddy itself.

\section{Sensitivity to particle releases}

We also test the sensitivity to how often we reseed the domain with particles. For 1 year of particle advection, we tested the sensitivity of the results to the frequency of particle releases. We released particles every 10, 20, and 30 days. Comparing these different releases, the more frequent releases show less noisy results. The rms value for 30 day particle releases is $3.09 \mathrm{~m}$, the rms value for 20 day particle releases is $2.14 \mathrm{~m}$ and the same value for 10 day particle releases is $2.41 \mathrm{~m}$. Because of this, we release particles every 10 days to be on the conservative side.

\section{Sensitivity to averaging period}

We also tested the role of how long to run the particle advection. We released particles every 30 days for 1 year and for 2 years. We compare the 1 year and 2 year output (Figure 4-10 and primarily see noise. Because of the lack of systematic change, we are convinced that 1 year is long enough.

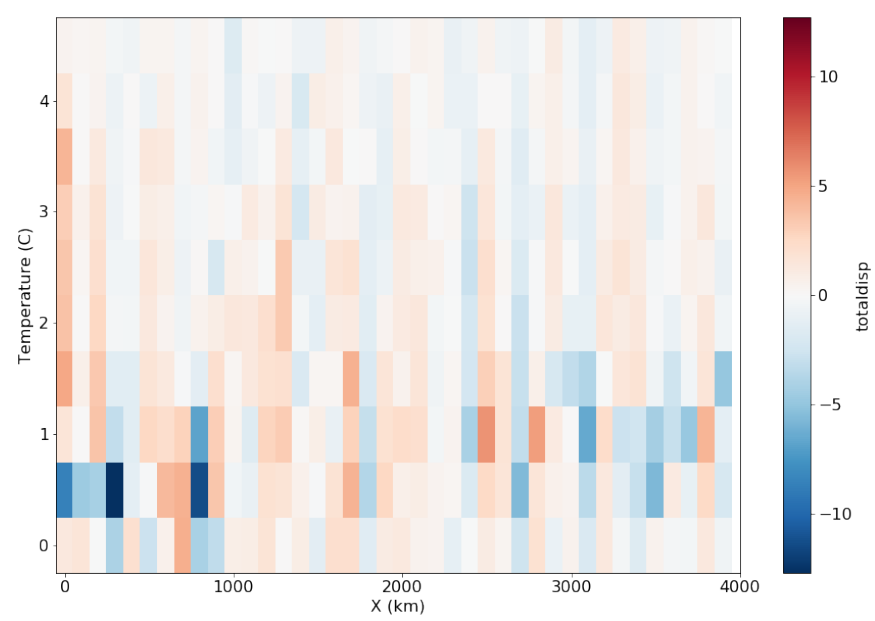

Figure 4-10: A figure showing the difference between 1 year of particle releases and 2 years of particle releases with particles released every 30 days, testing the length of the averaging period. The difference here is primarily noise, indicating that one year is sufficient for our purposes. 


\subsection{Results}

Our goal in this study is to understand the three-dimensional circulation of the Southern Ocean and what dynamical processes are leading to the vertical fluxes of water parcels and tracers. We analyze the vertical displacement of particles in order to understand the upwelling (Figure 4-6). We bin particles by their initial temperature in $0.5^{\circ} \mathrm{C}$ bins and map the displacement onto an $\mathrm{x}-\mathrm{y}$ map, averaging over all particles in each $\mathrm{x}, \mathrm{y}$ and $\mathrm{T}$ bin. From this, we take an average in $\mathrm{y}$. Using this we create maps of vertical displacement versus $\mathrm{X}$, which creates a map of the zonal asymmetries of upwelling.

We split up the domain into three different regions. The first is the upstream region between the entrance to the channel to the ridge at $800 \mathrm{~km}$. The next region we call the downwash region is between the topography at $800 \mathrm{~km}$ to about $1200 \mathrm{~km}$. Finally is the downstream region is between $1200 \mathrm{~km}$ and the end of the channel.

Generally we see an overall pattern of upwelling upstream of topography and downwelling in the downwash region. Most of this driven by the mean. In the downstream region there is mean upwelling in the cooler temperatures and mean downwelling in the warmer temperatures consistent with a Deacon cell, but is in fact due to steeping isopycnals in the downstream region. Generally the eddies are baroclinic eddies and acting in opposite direction to the mean flow. The barotropic eddies seem to be not important except near the bottom. Generally the upwelling is larger in the cooler temperature range in part because the flow is slower there so there is more time to upwell or downwell. To go into more detail, we split the flow into three temperature ranges based on where they are located in the overturning cell (Figure 4-4). These are lower cell downwelling, lower cell upwelling and upper cell upwelling. These results are shown individually in each cell in Figure 4-11. We do not analyze the upper cell downwelling because much of the upper cell downwelling occurs in the northern boundary region, preventing this type of analysis.

The alternate particle tracking method (Figure 4-7) shows a specific pattern of upwelling and downwelling following the particles as the cross the domain. First there is upwelling and then downwelling over the ridge, then there is enhanced upwelling or downwelling in the downwash region depending on the particle location, then the pattern reverses as the particles traverse the downstream region, showing that isopycnals are flattening then steepening as the particles travel downstream. 


\section{Lower Cell Downwelling}

The lower cell downwelling is dominated by the combination of the mean flow and baroclinic eddies (Figure 4-6 4-11). The mean flow leads to upwelling upstream of the topography, then downwelling in the downwash of the topography. Then downstream there is mean flow upwelling. The eddies act primarily to counter the mean flow leading to downwelling in the downstream region; the eddies act in opposition to the mean flow near the topography. In this downstream region, the wind is almost exactly balanced by the eddies.

\section{Lower Cell Upwelling}

Now we consider the upwelling branch of the lower cell (Figures 4-6 4-11). The lower cell upwelling is dominated by the mean flow upstream of topography and eddies in the downwash, so there is net upwelling near the topography. This shows us that the mean flow is important for the upwelling. Downstream of the topography, there is almost no upwelling or downwelling. Thus particles traversing the domain from west to east are first upwelled via the mean flow, then upwelled via eddies and then stay at the same level for the rest of the domain.

\section{Upper Cell Upwelling}

The upwelling in the upper cell is similar, except that the mean displacement upstream of topography is relatively weaker compared to the eddy upwelling in the downwash(Figures 4-6 4-11). There is downwelling driven by the mean in the downstream region, and it is almost exactly canceled by the eddy-driven upwelling leading to little net contribution to the upwelling downstream. Thus a particles traversing the domain would experience almost the same thing as in the lower cell, except the eddy-upwelling is stronger in the downwash region.

\subsubsection{Vertical Particle Motion}

Since the particles are generally maintaining their temperature, we can think of the vertical motion in isopycnal coordinates

$$
w=\frac{\partial}{\partial t} Z+u \frac{\partial}{\partial x} Z+v \frac{\partial}{\partial y} Z
$$


where $Z(x, y, t)$ is the isopycnal depth. The vertical velocity and displacement will have both contributions from the advection by the mean velocity against the mean gradient of the isopycnal depth and the eddy terms. The latter include dispersion, Stokes' drift, and bolus fluxes Gent and McWilliams, 1990. The experiments indicate that the mean and eddy fluxes are both important but in different regions, and that it is important to have a good representation of both.

\subsubsection{Mean Flow}

Since the mean flow seems to be very important for our understanding of the upwelling, in this section we will take a deeper dive into the mean flow. The particular structure of the mean flow is set by two different driving forces. First, there is the Deacon cell component due to the wind. The Deacon cell is the wind-driven overturning circulations bringing up cold water and down warm water Deacon, 1937, Marshall and Speer, 2012.

The other is the mean flow due to topography, which modifies the flow by diverting the flow first northward and then southward and up and down around the topography. Eddies can also play a role here by setting the structure of the mean flow. In addition, in the downstream region we see particles upwelling in the cool water range and downwelling in the warm water range, in the same direction as the Deacon cell. Since the Deacon cell effectively cancels out when we average in y, this is actually telling us about the mean isopycnal slopes downstream. This is telling us that the slopes are steepening downstream.

One possible way to distinguish these two components is by splitting up the flow into geostrophic and ageostrophic components. The geostrophic flow would primarily be due to the topography. The Deacon cell component is ageostrophic. As the wind changes we expect that these two different components will change. However, we know how the Deacon cell component will change, but not the primarily geostrophic component due to topography. This merits further investigation. Another way to distinguish the topographically-driven component from the wind-driven component is by taking a zonal average and the deviation from it to investigate the standing eddy component (the zonal deviation from the timeaveraged flow). 


\section{Comparison to Simple Models}

Flow over topography has been studied extensively in meteorology often with a view toward local lee-wave generation, but also on larger scales examining Rossby wave generation [e.g. Charney and Eliassen, 1949]. Such investigations into the stationary waves have often dealt with topography which is low wavenumber and not too different from the stationary wave scale; thus the periodicity is important. For our problem, however, the width of the ridge is small compared to the channel length, and we don't see obvious global modes. The steady pattern from the topography is perhaps better thought of as a case with specified inflow. In this case, as Charney and Flierl 1981 point out, there is no resonant response even on the beta-plane. Thus the simplest paradigm - barotropic flow on an f-plane - illustrates what is expected. In the southern hemisphere, as the flow, which conserves $(f+\zeta) / h$, its absolute vorticity becomes less negative, so that the vorticity is positive over the ridge. This implies that northward flow, growing rapidly as the ridge is approached and more slowly as the water descends. Beyond the ridge, the northward flow will begin to turn south in a standing Rossby lee-wave pattern.

If the flow is weak enough or beta strong enough, the flow will come closer to following $f / h$ contours and turns back southward on the eastern flank of the ridge. The degree to which the flow becomes southward again depends on the ratio of the Rossby wave speed to the upstream flow $\beta L^{2} / U$. In any case, the upwelling and downwelling patter is determined by the ridge itself as the water shallows and the deepens.

The stratified problem is more complex, even with the quasigeostrophic approximation. but has a similar character. For fast flow, the motions decay in the vertical, while slow flow can lead to something more like the baroclinic mode. Other phenomena, such as internal wave generation and blocking below the peak of the ridge, become possible when the full equations are considered.

These steady solutions tend to have wave instabilities; in the stratified problem, too, baroclinic shears develop as the different layers respond more or less strongly to the topography. Even when the incoming flow is stable, then, we expect the flow will become unsteady and turbulent as we see in the simulations. But the basic pattern of upwelling and downwelling associated with the mean flow is broadly in line with expectations from the simple models. 


\section{Future Work}

To further investigate the role of the mean flow, we believe a more quantitative analysis of whether or not the mean topographically-induced flow is leading to vertical transport of tracers. We see this being some kind of enstrophy budget or the like. We will also investigate how the mean flow changes with the wind.

\subsubsection{Comparison to Flat-bottomed Ocean}

In order to understand how our analysis for the simulations with the ridge are different than the traditional flat-bottomed picture, we analyze the flat-bottomed simulations. We can compare these results (Figure 4-12) to the zonally-averaged residual overturning (Figure 4-4). We expect to see a wind driven overturning going one direction, with upwelling in the south and downwelling in the north in the mean and eddies counteracting this, however what we see is more complicated (Figure4-12). We actually see the wind driven overturning as expected in the mean, and a more complicated pattern dominated by near-ish surface upwelling.

We are not averaging in $\mathrm{y}$ for the flat bottom here because the wind driven overturning cancels out when we take the average. It also cancels out in the ridge case, what we actually see in the ridge case is the isopycnal slopes changing in the mean.

The reason we can see structure more easily in the ridge case is that the signal is relatively larger than the noise in the ridge case. In addition it seems like the short duration particle advection doesn't represent the flat-bottomed flow very well. When we advect particles in the long time following particles, we get the representation we expect of the deacon cell versus eddies.

From the discussion of the flat bottom, we can derive insights into the ridged simulation. Because of the forcing, we are not changing the magnitude of the overturning or the zonalaverage structure of the overturning, but we are instead changing the location of the action and the depth structure. Note that the magnitude of the overturning could change slightly because Ekman pumping into a layer can be different, as can the heat flux due to the meandering, as well as the horizontal buoyancy gradient. Figure 4-4 shows little change in the magnitude. The depth structure changes because the isopycnal slopes are significantly flatter with the ridge, indicating that the topography is making the baroclinic extraction of 
available potential energy much more efficient. This shoals the location where the isopycnals meet the northern boundary (or the interior of the ocean). For the ridge case, the action is also isolated to specific areas, primarily near the topography. While the wind driven overturning doesn't change between the flat bottom and the ridge simulations, the timemean (standing eddy) and the transient eddy terms are highly localized.

\subsubsection{Changes with Wind}

We are also interested in understanding how the flow will change with changing wind stress, inspired by the changes that climate change are making to the atmospheric circulation Marshall, 2003|. We see that as we increase the wind, the peaks near topography become taller and narrower (Figure 4-13). The mean component becomes more important in all layers. In addition, there is suppressed eddy-driven transport downstream, in agreement with suppressed eddy activity seen in $\|$ Youngs et al., 2019 and others. As a result, the mean is entirely driving the flow downstream. A further analysis into how the overturning varies with the wind will be undertaken for the published version of this work.

\subsection{Discussion and Conclusions}

Near topography, we see enhanced upwelling on the upstream slope of topography (Figure 4-14). We speculate that this is primarily adiabatic geostrophic flow. In the downwash region, we see an enormous eddy potential energy release in terms of flattening isopycnals, also seen in Youngs et al. 2017] (Appendix B). This leads to the question of why there is enhanced eddy activity downstream of the topography. We speculate that this is due to several reasons including an elimination of the stabilizing effect of a horizontal shear due to the initiation of barotropic instability as well as the destabilizing effect of the flow turning meridionally and traversing topography Youngs et al., 2017](Appendix B).

In chapter 3, we saw isolated eddy activity downstream of the topography, including upgradient heat fluxes. This combined with the energy budgets of Youngs et al. 2017] (Appendix B), shows that eddies in the downstream region are adding to the available potential energy. We can see this in this chapter by looking at the displacement of particles which indicates a change in isopycnal slopes, showing how the eddies modifying the mean flow are working in concert with the wind. Chapter 3 is different because it dealt with single 
cells at a time, which made us unable to see the full behavior of the isopycnal slopes.

In this study, we show what forces drive vertical transport in an idealized Southern Ocean channel (Figure 4-14). Mean flow is especially important. Downstream of topography in the wash zone, baroclinic eddies counter the tendencies of the mean flow, but primarily leading to upwelling. As the winds increase, the contribution due to topography from mean and eddies becomes larger and eddy activity is minimized downstream in agreement with chapter 3 and appendix B. We filled the gap by improving our understanding of the localized and de-localized nature of the overturning.

We have discussed how the mean and eddies shape where the movements up and down the isopycnals occur. Although we see clear differences in the flows in the experiments with different wind strengths, there remain many interesting questions about the physics which results in the mean structures, the distribution of eddy energy, and eddy fluxes. The energetics study Youngs et al. [2017] in Appendix B certainly argues that the instability processes and the feedback between the eddies and mean is significantly impacted by the ridge. It seems quite likely that the paradigm of temporally growing waves on a zonal flow may not be applicable. There appear to be regions in which the eddies are gaining energy and others in which they are losing it, as in Waterman and Jayne, 2011. The eddies in the downwash region seem to be developing both spatially and temporally, perhaps as a manifestation of a "convective" instability starting as the jet intensifies as it veers northward over the ridge. In short the dynamics leading to the pattern of the residual mean $w$ remain a topic worthy of much more extensive study.

There are several limitations of this study. First, Lagrangian particles are not the same as residual circulation, however we believe we can use this because we expect it to be not that different. Another limitation is that the actual processes driving the lower cell are not represented in detail in this model, since the lower cell is thought to be driven by dense overflows off of ice shelves, whereas we have more distributed cooling acting to increase the density. However, this study focuses on the interior of the ocean, regardless of how the dense water was formed.

Our strength is the new angle investigating this problem. This highlights the processes we need to resolve in models and where we need to sample to understand overturning. Models need to be run at higher resolutions around topography because these regions are critical. The $200 \mathrm{~km}$ around our topography are of major importance for the flow. We 
have shown that much of the upwelling is driven by both the mean flow, upstream of the topography and the eddies just downstream of topography, highlighting the importance of localized measurements to appropriately capture the Southern Ocean overturning. 

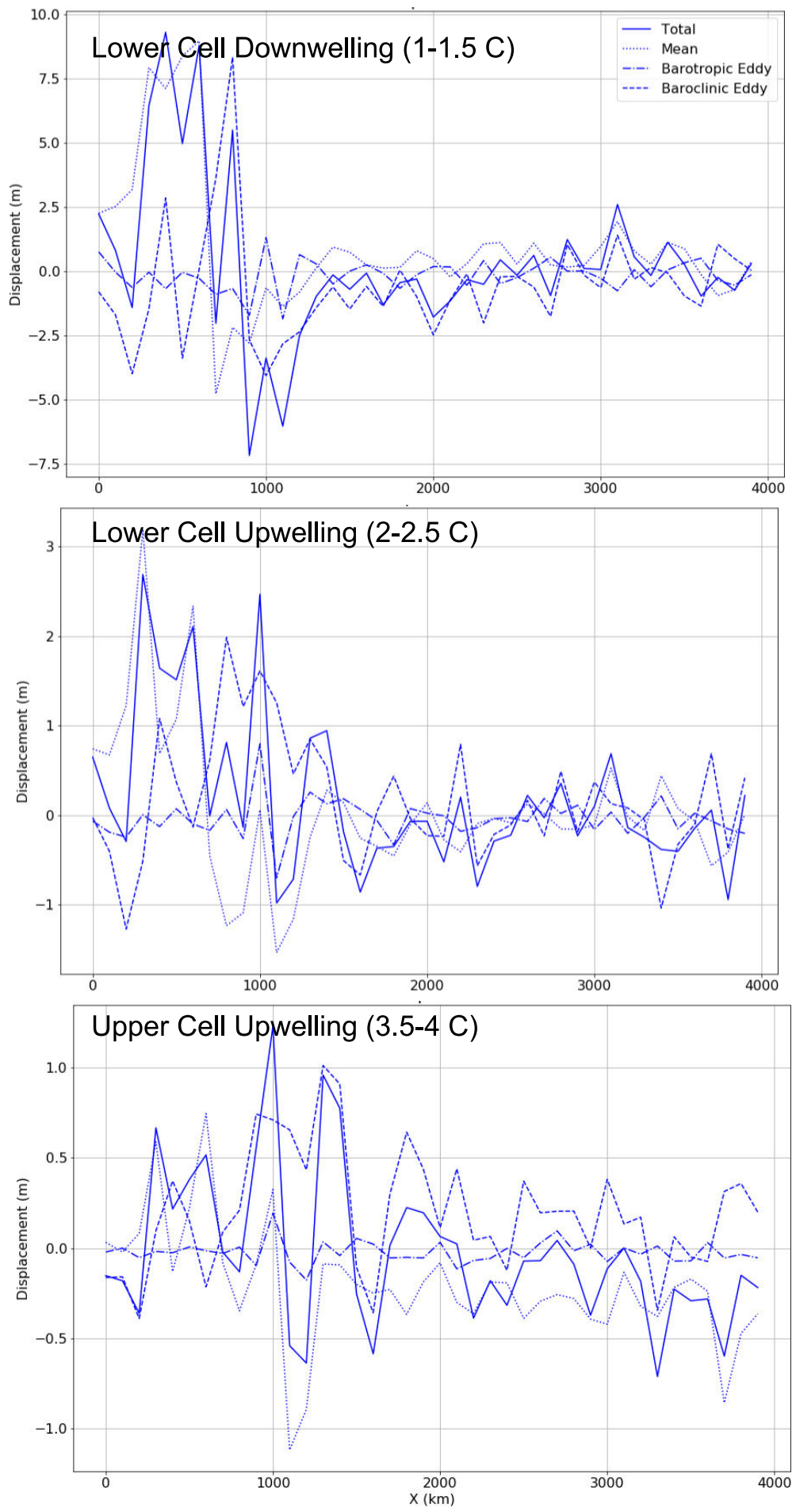

Figure 4-11: Displacement versus X split among different components for different temperature bins that represent the different overturning cells. This displacement is the amount particles upwell in the box, a kind of weighted velocity that weights how long a particle spends in each cell. We generally see upwelling before the ridge at $800 \mathrm{~km}$ and downwelling after. 

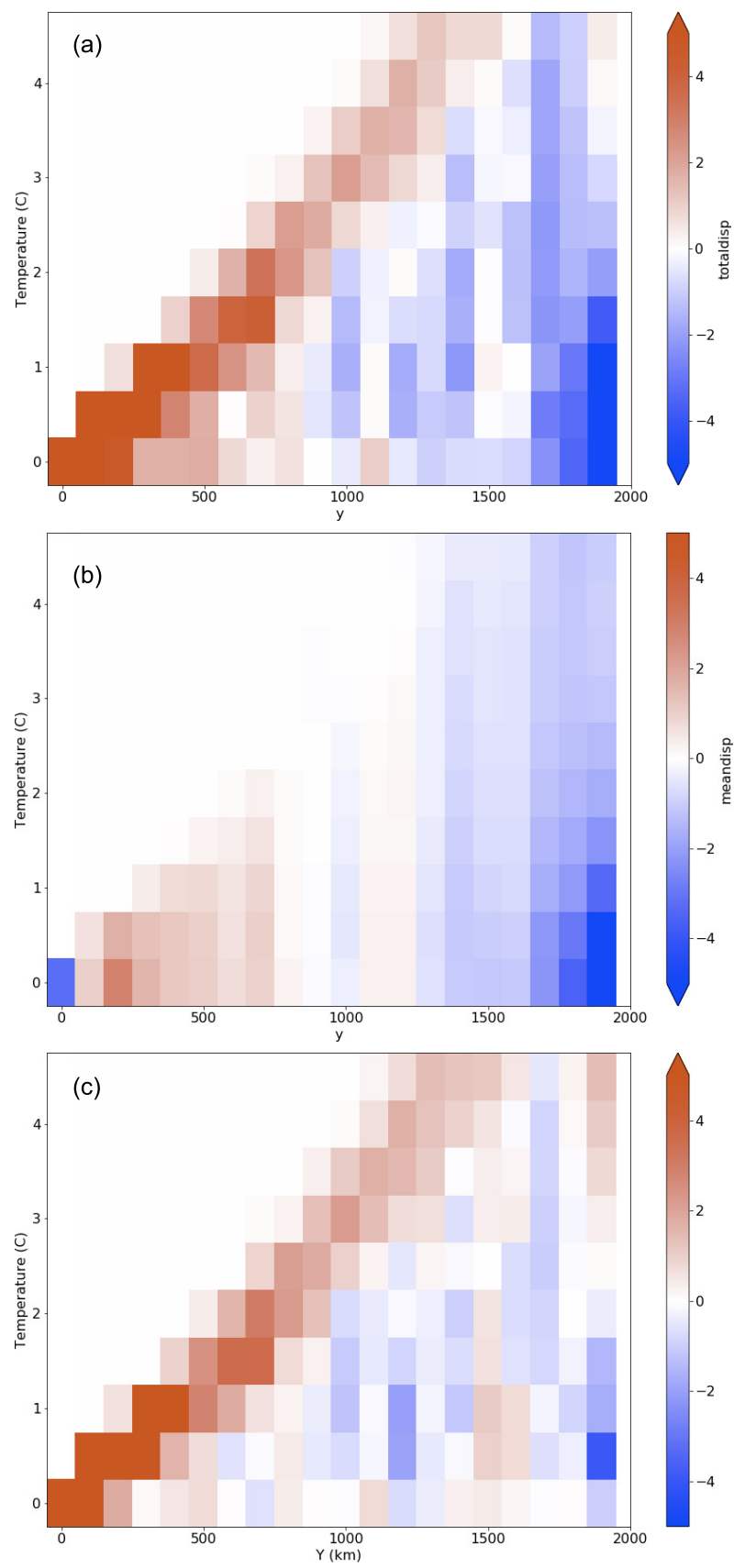

Figure 4-12: Zonally-averaged displacement of particles in a flat bottomed channel where $\tau_{0}=0.15 \mathrm{~N} \mathrm{~m}^{-2}$ in $\mathrm{m}$. (a) shows the total vertical displacement (b) shows the mean, and (c) shows the eddies. 

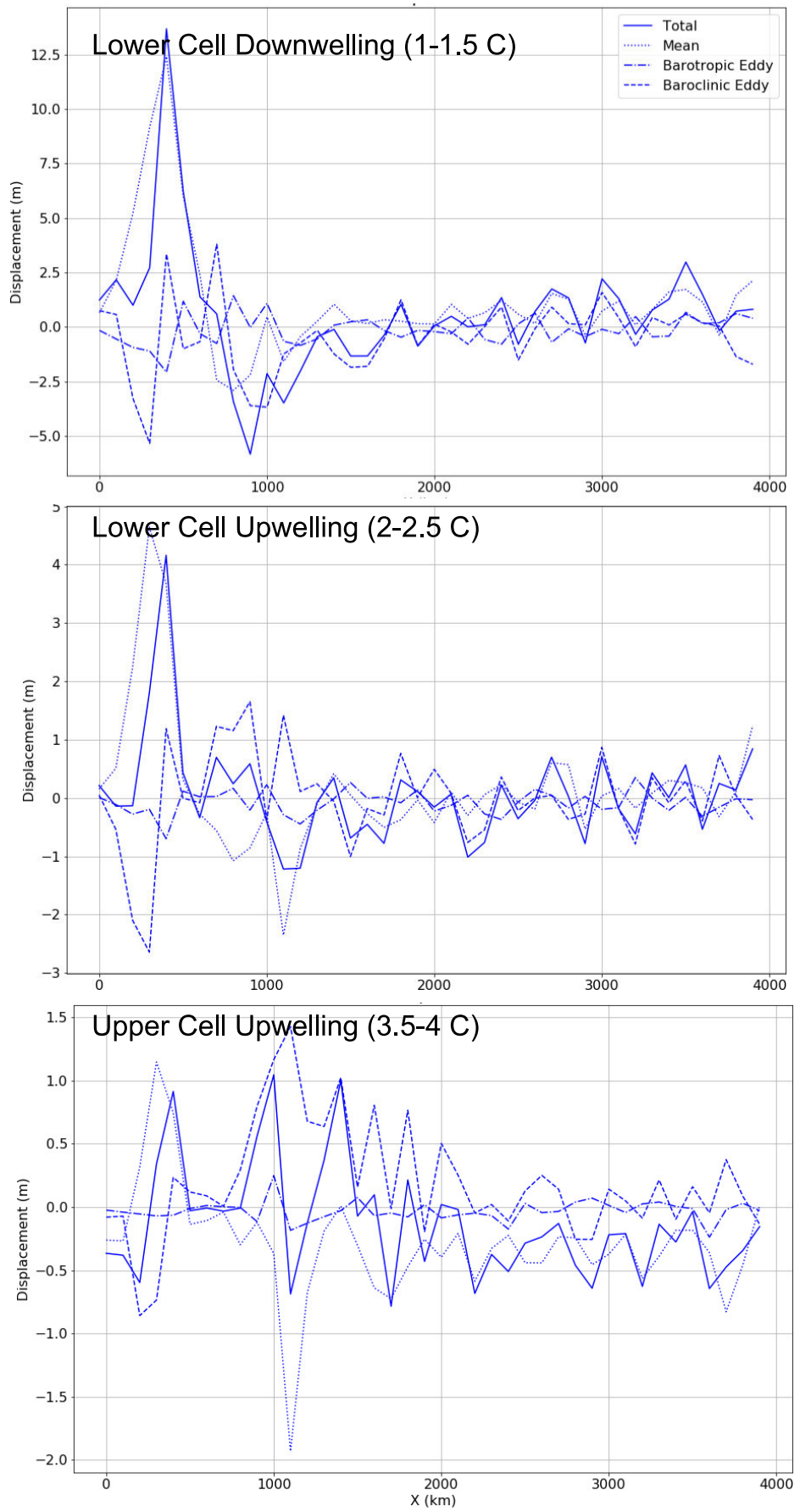

Figure 4-13: Just like figure 4-11 except for $\tau_{0}=0.2 \mathrm{~N} \mathrm{~m}^{-2}$. Notice that baroclinic eddies are significantly weaker downstream for the stronger wind. 


\section{Old Understanding}

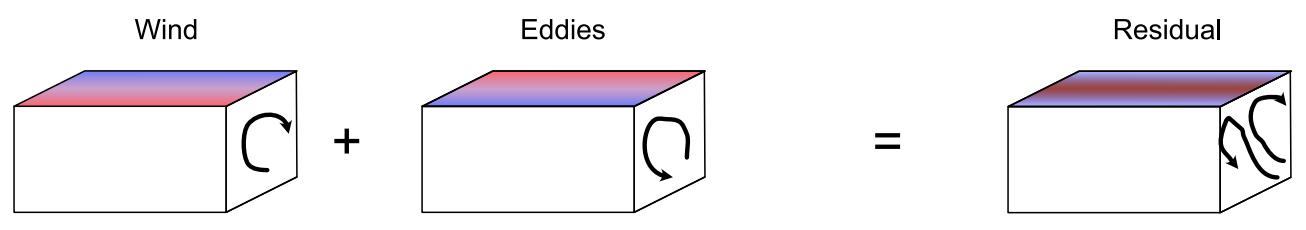

\section{New Understanding}

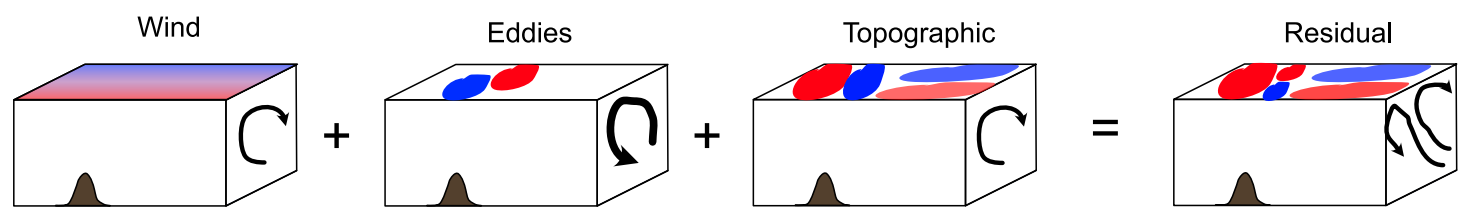

Figure 4-14: A figure schematically comparing our old understanding of the overturning to our new understanding. Instead of just wind and eddies, there is now a topographic component and the eddies are localized. The topographic component, also called standing eddy component, is working in concert with the wind in the downstream region of topography. Note here that in our simulations the upper cell downwelling occurs entirely at the northern boundary, so we don't represent these processes in the schematic. 


\section{Chapter 5}

\section{Localized Air-sea Carbon Fluxes due to Topography in a Southern Ocean-like Channel}

\subsection{Introduction}

The Southern Ocean comprises over $30 \%$ of the global ocean area but it takes up about $50 \%$ of anthropogenic carbon Majkut et al. 2014, Gruber et al. 2019]. However, this result is not well constrained, due to a lack of areal coverage of data, specifically in winter-time. With the biogeochemical floats released via the SOCCOM project, the data available has increased rapidly.

One uncertainty stems from regional variability of air-sea carbon fluxes Sallée et al. 2012. Dynamically, there are standing meanders that dominate the lateral fluxes of heat and carbon [to et al. 2010, but we still don't know how these relate to total air-sea fluxes. On top of that, the fluxes can be dominated by local biological processes [e.g. McElroy, 1983; Uchida et al. 2019; Ardyna et al., 2019. So we need to understand how local processes affect total air-sea carbon fluxes as well.

One proposed reason for the local hotspots of carbon fluxes is the local upwelling. Physically, numerical modeling has also shown that there are regions of enhanced upwelling near the topography, suggested to be due to mesoscale eddies [Tamsitt et al., 2017]. We have shown in the previous chapter that the topographic-enhanced upwelling is due to eddy ac- 
tivity as well as the mean flow. From this result, the connection between the enhanced upwelling and the carbon budget needs to be made. We endeavor to make this connection in this chapter.

We connect the physical circulation of the Southern Ocean to localized air-sea carbon fluxes using an idealized channel model, developed in Chapter 4. The simplified geometry and biogeochemical model allows us to make a dynamical link between the topographic circulation and the carbon. In section 2 we talk about the model configuration and in section 3 we discuss the analysis of the mean state. In section 4 we discuss the response of the system to changes in wind.

\subsection{Methods}

In this chapter, we build off the work of Chapter 4 by adding a biogeochemical model to the MITgcm channel used in that study. The channel is $4000 \mathrm{~km}$ long by $2000 \mathrm{~km}$ wide with 10 $\mathrm{km}$ resolution. While this resolution is similar to the deformation radius of $15 \mathrm{~km}$, the main scale of mesoscale motion tends to be larger than the deformation radius Pedlosky, 1987. We have a total depth of $4000 \mathrm{~m}$ with 32 points in vertical, from $10 \mathrm{~m}$ grid spacing at the surface to $\mathrm{n}$ meters in grid spacing at the bottom. For topography we have a Gaussian ridge of half-width $200 \mathrm{~km}, 2000 \mathrm{~m}$ tall, located at $800 \mathrm{~km}$ downstream of the channel entrance. We use a 600 second time step, free slip sides, and a diffusivity varying from $0.01 \mathrm{~m}^{2} \mathrm{~s}^{-1}$ to $1 \times 10^{-5} \mathrm{~m}^{2} \mathrm{~s}^{-1}$ exponentially. We also have a linear bottom drag with a drag coefficient of $1.1 \times 10^{-3} \mathrm{~m} \mathrm{~s}^{-1}$.

We take my MITgcm channel model from a previous chapter, then add the DIC package from MITgcm Lauderdale et al. 2016. This is a biogeochemical model that models biological growth simply by limiting growth by light and nutrients. $S_{b i o}$ represents the sources and sinks of biogenic soft tissue and $\mathrm{S}_{\mathrm{CaCO}_{3}}$ represents the sources and sinks of biogenic carbonate. The nutrient here is phosphate, which is fluxed vertically with remineralization and sinking. There are 4 biological variables calculated as tracers: $\mathrm{pH}$, alkalinity, dissolved inorganic carbon, and dissolved organic phosphorus. Neglecting the dilution by freshwater fluxes, we have the following equation:

$$
\frac{\partial C_{T}}{\partial t}=-\nabla \cdot\left(\vec{u} C_{T}\right)+\nabla \cdot\left(\kappa \nabla C_{T}\right)-R_{C_{T}: P} S_{b i o}-S_{C a C O_{3}}-\frac{F_{C O_{2}}}{h}
$$


where $C_{T}$ is the concentration of total dissolved organic carbon, $\kappa$ is the eddy diffusivity tensor, $R_{C_{T}: P}$ is the biological transformation between carbon and phosphorous and $F_{\mathrm{CO}_{2}}$ is the air-sea $\mathrm{CO}_{2}$ fluxes and $h$ is the mixed layer depth. The biological parameters set are a light attenuation $k_{0}$, timescale for biological activity $\alpha$, half saturation phosphate constant, and an inorganic/organic carbon rain ratio. The light attenuation is calculated as

$$
l i t=e^{-k_{0} z}
$$

The constant used by default is $k_{0}=0.021 / \mathrm{m}$. Biological growth is co-limited by light and nutrients.

$$
S_{b i o}=\alpha \frac{l i t}{l i t+l i t 0} \frac{P_{4}}{P O_{4}+K_{P O_{4}}}
$$

and

$$
S_{\mathrm{CaCO}_{3}}=\frac{1}{2} \text { rain_ratio } R_{C_{T}: P} S_{b i o}
$$

If phosphate is fluxed to the bottom, it is instantly remineralized.

\begin{tabular}{c|c|c} 
symbol & meaning & value \\
\hline$k_{0}$ & light attenuation coefficient of water $[1 / \mathrm{m}]$ & 0.02 \\
$\alpha$ & timescale for biological activity $[1 / \mathrm{s}]$ & $2 \cdot 10^{-3} /(360 \cdot 86400)$ \\
$K_{P O 4}$ & half saturation phosphate constant $\left(\mathrm{mol} / \mathrm{m}^{3}\right)$ & $5 \cdot 10^{-4}$ \\
rain_ratio & inorganic/organic carbon rain ratio & $7 \cdot 10^{-2}$
\end{tabular}

Table 5.1: Biological parameters used in the MITgcm simulation and their values.

For boundary conditions, we set up profiles at the northern boundary to relax the DIC and Alk using data from GLODAPv2 Key et al., 2015, Lauvset et al., 2016. We set the surface value of $\mathrm{pCO}_{2}$ to be $270 \mathrm{ppm}$. We spin up the carbon model for 30 years for the biogeochemical and physical components to equalize. See table 5.1 for values of coefficients. We used an estimate of net pre-industrial air-sea $\mathrm{pCO}_{2}$ flux from Lovenduski et al., 2007. to verify the configuration has an appropriate magnitude of total air-sea carbon fluxes over the entire Southern Ocean.

\subsection{Analysis of Mean State}

These simulations provide insight into understanding the role of topography in driving carbon fluxes by simplifying the system to an understandable level. In this section we will 


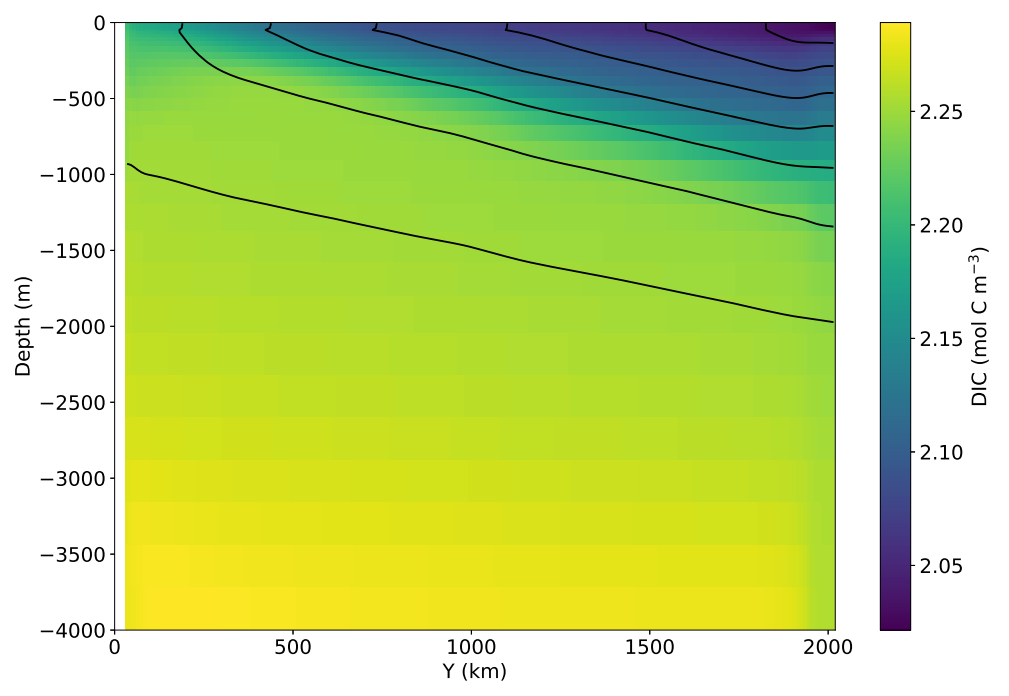

Figure 5-1: A figure showing a DIC section for $\tau_{0}=0.15$. Note the difference between the northern boundary and the interior. Black contours show temperature contoured every degree. At the northern boundary we relax to GLODAPv2 preindustrial DIC.

address the following questions: Why is there outgassing in the southern part of the domain and uptake in the northern part? Why is there outgassing in the meander?

Figure 5-2 shows the air-sea $\mathrm{CO}_{2}$ fluxes at the sea surface. In this case, red is outgassing and blue is ingassing. This shows us that outgassing is important generally in the southern part of the domain, also with a broad peak over topography. The general peak at the southern part of the domain indicates the importance of upwelling for the domain. To investigate why there is more outgassing over the topography, we use a linearization to look at the contribution of different mechanisms for the air sea carbon fluxes by comparing a weak wind to the base case wind stress in the next section.

To answer these questions we will take the following approach. First, we aim to quantify the role of DIC for outgassing in the mean state. From this point we will look at a DIC budget quantify the role of mixing, advection, biology, etc. We will do this analysis in the coming months after the defense. 


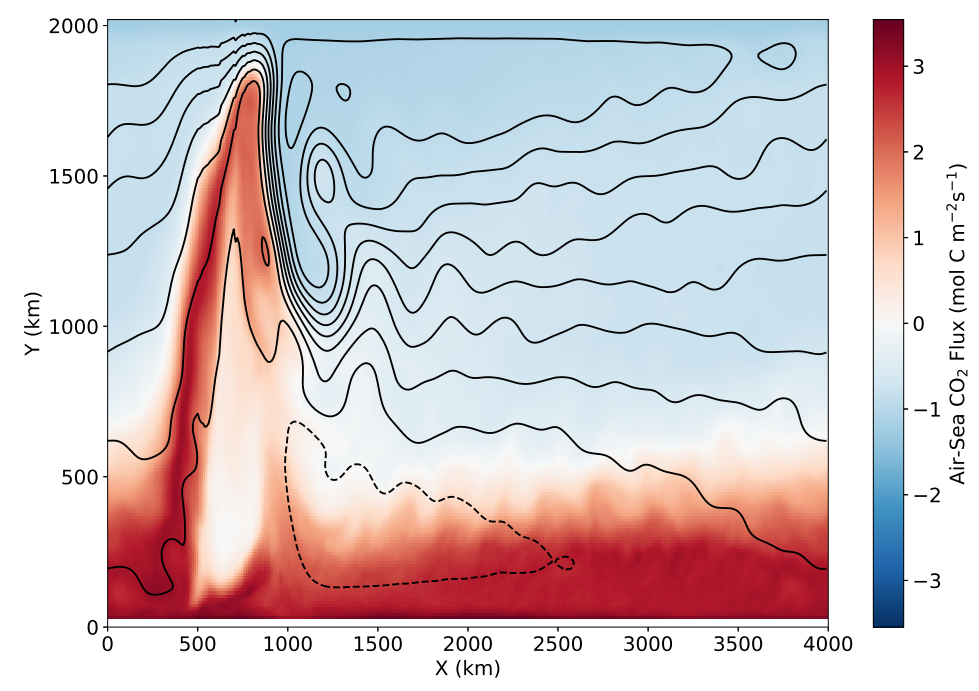

Figure 5-2: Air-sea carbon fluxes with contours showing the barotropic streamlines. The boxes indicate the regions where we compute the difference in $\mathrm{pCO}_{2}$, used in a previous analysis.

\subsection{Analysis of Perturbation Due to Wind}

We also investigate how the air-sea carbon fluxes change as we change the maximum wind stress (Figure 5-3). As we increase the wind stress, there is more outgassing, switching from a net carbon uptake to a net outgassing over the wind range. The increase in the outgassing is linear for the weaker wind stresses, but slows as the winds become stronger, indicating a saturation in the system of some sort, potentially dynamically.

We have calculated Equation 2 from Lovenduski et al. [2007] comparing the base case wind to the weakest wind.

$$
\Delta \mathrm{pCO}_{2}=\frac{\partial \mathrm{pCO}_{2}}{\partial D I C} \Delta D I C+\frac{\partial \mathrm{pCO}_{2}}{\partial A l k} \Delta A l k+\frac{\partial \mathrm{pCO}_{2}}{\partial T} \Delta T
$$

We use a simple linear $\mathrm{pCO}_{2}$ solver to calculate the $\mathrm{pCO}_{2}$ from the $\mathrm{DIC}$, Alk, and temperature values. We then subtract out the base case wind $\tau_{0}=0.15 \mathrm{~N} \mathrm{~m}^{-2}$ from the weakest wind $\tau_{0}=0.05 \mathrm{~N} \mathrm{~m}^{-2}$. This combination was chosen at random, others could be investigated as well.

We see that change in total $\mathrm{pCO}_{2}$ fluxes has a definite pattern (Figure 5-4). Blue indi- 


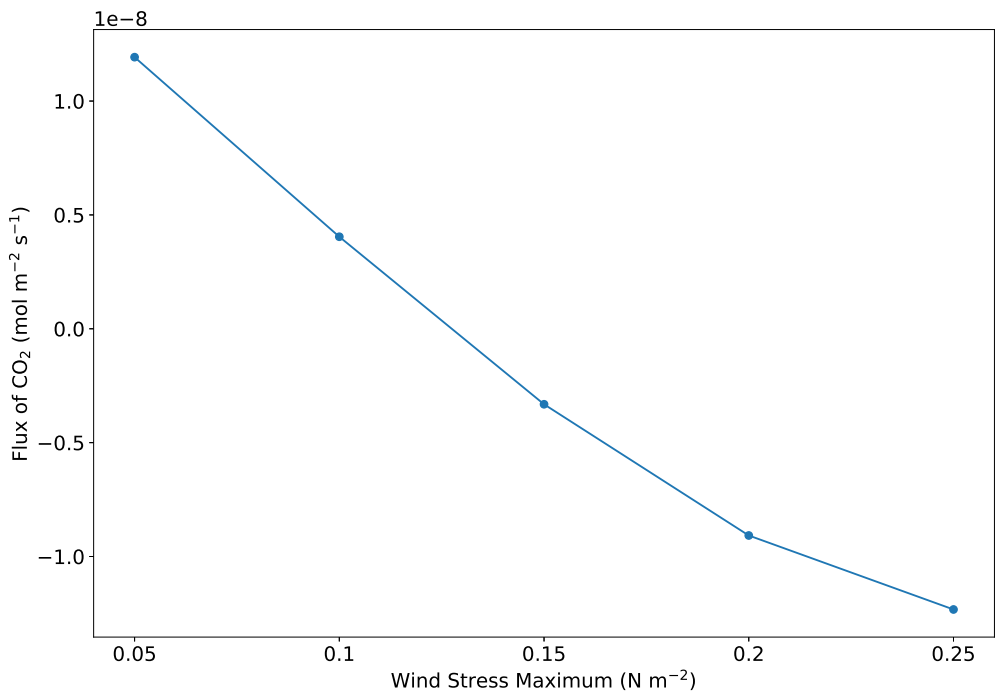

Figure 5-3: Figure showing the air-sea carbon fluxes averaged over the whole domain versus the wind stress maximum.

cates that there is more outgassing with the stronger wind, which is elevated near the trough of the meander (the top of the ridge), and lower elsewhere. This seems to be dominated by the changes in DIC, indicating advection of DIC as a major driving component. The Alk term seems to mitigate the changes from DIC mainly in the meander, and it also contributes to the sign in the downstream region of the domain. The temperature contribution seems to be negligible.

\subsection{Discussion and Conclusions}

In this chapter, we show that topography and its resulting circulation changes lead to enhanced outgassing near topography. As the wind stress increases, the flux increases near the topography due to changes in DIC, pointing to changing advection patterns behind the enhanced outgassing. Our study allows for a more detailed look at how carbon cycles through the Southern Ocean due to the model's idealized nature.

The model's idealized nature is also a potential pitfall due to its simplified geometry and the simple model for the biochemistry. We use this as an advantage to create a framework to understand more complex models as well as observations, concretely clarifying our 

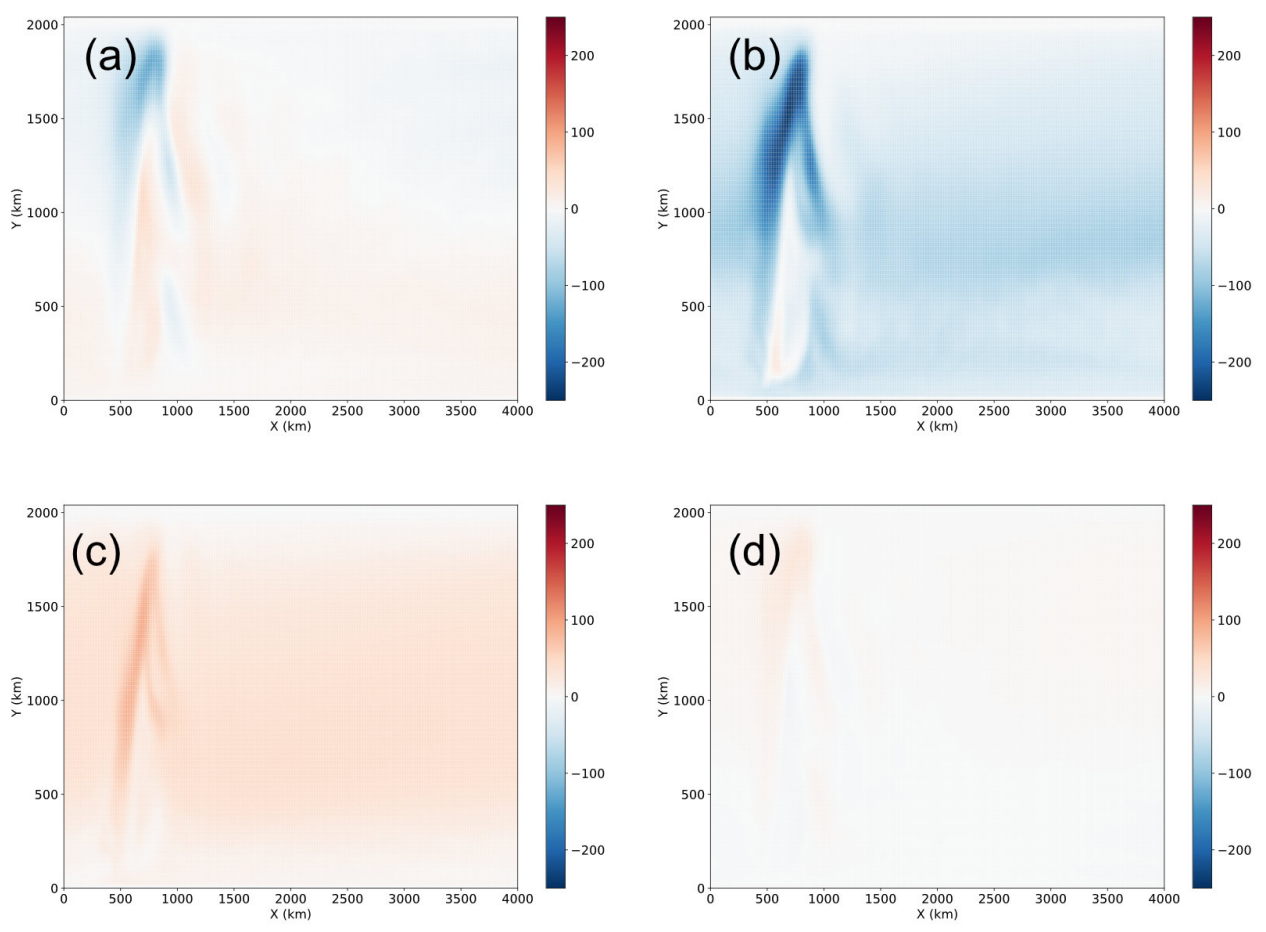

Figure 5-4: Figure showing the contribution of different terms to air-sea carbon flux differences (weak wind $\left(\tau_{0}=0.05 \mathrm{~N} \mathrm{~m}^{-2}\right)$ - moderate wind $\left(\tau_{0}=0.15 \mathrm{~N} \mathrm{~m}^{-2}\right)$ ) in $\mu$ atm. (a) shows the total difference in $\mathrm{pCO}_{2}$ fluxes, (b) shows the contribution from DIC, (c) shows the contribution from Alk, and (d) shows the contribution from temperature.

understanding of how Southern Ocean carbon will change with climate. 


\section{Chapter 6}

\section{Conclusions}

In this thesis, I have explored the role of zonal asymmetries for controlling the dynamics of overturning circulation. In this section I will discuss how my thesis fits into the literature and how it has advanced the field. I will also conclude by discussing some of my perspectives on the field as a whole, including what are the most important questions to address, challenges to addressing these questions, and infrastructure necessary to address these challenges.

\subsection{Place of this thesis in the literature}

\subsubsection{Basin-Width Dependence of Northern Deep Convection}

In the first chapter of this thesis, I developed a simple scaling law to estimate the impact of freshwater fluxes on high latitude convection. The proposed mechanism only depends on the asymmetry of basin width and fundamental scaling laws, unlike many other theories that depend on asymmetries of continent length Jones and Cessi, 2016, freshwater catch basins [Ferreira et al., 2010], differences in wind-driven gyres [Jones and Cessi, 2017]. The previously proposed theories are quite complex and sometimes difficult to understand. This mechanism is simple but useful because it explores the role of salinity for deep convection. This simplicity allows the scalings to be generalizable to a wide range of paleoclimate scenarios and boundary conditions.

Because of the simplicity, follow up work would be required to show the relative importance of our mechanism versus other mechanisms and could be undertaken using more realistic models. A method to quantify the relative importance of various mechanisms would 
need to be developed, and it is quite possible that the mechanisms are not independent of each other but instead feed off of each other. In fact, I suspect that our mechanism interacts with interbasin salt transport mechanisms based on my interpretation of our two basin simulations.

\subsubsection{Role of Residual Overturning for the Sensitivity of Southern Ocean Isopycnal Slopes to Changes in Wind Forcing}

In my next chapter, we studied the role of topography in setting how the isopycnal slopes change in the Southern Ocean with a change in wind. We model the Southern Ocean using a two-layered quasi-geostrophic model, our simplest channel model. Understanding here has been developed for a flat bottomed channel with out any heat fluxes, which lead to the development of the idea of eddy saturation. This thesis has applied a technique to model the residual overturning circulation utilized in the atmospheric literature, in order to understand the role of the overturning. It has also highlighted the way that isopycnal slopes change with wind, showing how important it is to continue studying the local instabilities downstream of topography. In addition, we show that heat fluxes are also necessary to show the dominant behavior. The slope changes also have implications for which water masses outcrop at which latitude, affecting the ventilation and fluxes experienced by each water mass.

The results of this study leave a few open questions: What is the role of the transient eddies in setting the mean state of the flow? Are the transient eddies a result of local or global instability? Why does eddy saturation occur and at what value of the transport will the flow saturate? To address some of these questions, I hope to explore a novel method to isolate the role of the transient eddies on the mean state of the flow. This method works by calculating the fixed points in phase space, or the (unstable) steady state solutions without any turbulence and allows for the analysis of the steady state flow.

\subsubsection{Causes of Localized Upwelling in a Southern Ocean-like Channel Near Topography}

The idea of residual circulation in a zonally-symmetric channel has dominated the field and brought many insights, however we show the importance of the topography for localizing the upwelling pathways. For the study of the residual overturning circulation and how it varies with wind locally, we built off the work of Abernathey et al. 2011 and Youngs 
et al., 2017. Ours is like Abernathey et al., 2011 except with a ridge, in order to more accurately represent the Southern Ocean. Our results have advanced the field because they describe what processes are driving localized upwelling. These processes likely include geostrophic uplift, highly localized eddy activity, and steepening isopycnals working with the wind-driven overturning. This is fundamental for understanding the current and future air-sea carbon fluxes and changes in the dynamics, both physical and biogeochemical. The technique that I developed is newly applied to the Southern Ocean problem.

Next steps here are to run a more realistic model (for example SOSE at $1 / 12^{\circ}$ ) and do a similar analysis to understand how our results apply to the real ocean and maybe an analysis of real ocean data too. I intend to keep studying this because of the relevance of this system for carbon and climate.

\subsubsection{Localized Air-sea Carbon Fluxes due to Topography in a Southern Ocean-like Channel}

The next chapter connects the physical circulation of the Southern Ocean to localized air-sea carbon fluxes using an idealized channel model [Youngs et al., 2017]. The simplified geometry and biogeochemical model allows us to make a dynamical link between the topographic circulation and the carbon. We added to the literature by highlighting the mechanisms of air-sea carbon fluxes in this idealized framework.

We find that there is enhanced outgassing of $\mathrm{CO}_{2}$ fluxes near topography for the lower cell of the overturning circulation and that this is primarily driven by enhanced upwelling near topography. If we properly represented the upper cell with mode water formation, we would also expect to see enhanced downwelling localized near to the topography. This enhanced uptake of anthropogenic carbon and outgassing of natural carbon near topography point to the necessity of properly sampling to observe the carbon system. Right now carbon outgassing and uptake estimates are likely too small because of the location of the instruments. The regions of highest outgassing are regions where the biogeochemical Argo flats would move through quickly, undersampling these regions. In combination with the small number of samples, there is a question of whether the shear is so large that the floats are not well following the water involved in the exchange. This points to the necessity of steerable instruments to sample these regions, such as wavegliders, gliders, and research vessels because Lagrangian (quasi-Lagrangian) instruments would not sample these highly 
important regions.

The next steps in this project are to connect the idealized modeling to the real ocean by comparing to a more realistic model, such as SOSE. I also propose to look at observational datasets and see if they are broadly consistent with our idealized results. After this analysis, ship time would be necessary to take measurements and deploy instruments.

\subsection{Perspectives}

\subsubsection{Important Questions}

What is the role of the ocean in climate change? Climate change is a fundamental threat to human life. I believe that because of this, we as oceanographers need to do our part to better understand the system and inform policy makers. The ocean plays a major role in moderating climate change by absorbing heat and carbon dioxide, but it also plays a role in melting glaciers. Thus, as oceanographers, we have many questions to investigate. I discuss some of the most relevant questions below.

How quickly will Greenland and the West Antarctic Ice Sheet collapse? How quickly the Greenland and West Antarctic Ice Sheets collapse will impact how quickly societies must adapt to changes in sea level. The ocean seems to be playing a dominant role for the melting of the Greenland and the West Antarctic Ice Sheets with warm ocean water melting floating ice tongues, leading to glacier collapse. This means that we need to constrain and predict the circulation and melting and how they are going to change over time. There is an oceanographic question of how does warm water get in underneath the ice shelves, as well as many questions about the dynamics of glaciers. The community seeks to understand the system and improve our modeling capabilities, so that we can provide better estimates for how big a sea wall is needed in New York City or Boston in the next 50 years. Another large unknown is how the ocean's reservoir of carbon dioxide and its exchange with the atmosphere will respond to climate change.

How does Southern Ocean circulation affect the future global carbon budget? The Southern Ocean plays an outsized role in the global carbon budget for it is the location where water takes up human-generated carbon dioxide and then sucking it down for a thousand years. At the same time, water is brought to the surface where really old carbon dioxide is exposed and released to the atmosphere. We need to understand both the uptake and 
draw down of carbon dioxide will change in the Southern Ocean and contribute to increasing carbon dioxide levels and warming temperatures.

There are many different processes controlling the carbon exchange, including large processes like the overturning circulation, and small processes like sub-mesoscale turbulence, and mixing, eddies, and even biological communities. We must investigate each of these different processes and learn how they will change with changing climate.

\subsubsection{Challenges Facing the Community}

Lack of thoughtfulness in funding decisions and data collection There are many reasons to do science, but whatever reason is why we do it, we need to be thoughtful about how our studies reach our goals. Throughout my career, I have seen a general lack of thoughtfulness in choosing scientific questions and methods. Much research has been done because it is just the next step of the previous research that has been done, but not that that research fits into the broader goals of the scientist and the scientific community as a whole. This leads to wasted time and money in the community. I think that this could be aided by having scientists from many disciplines scrutinizing studies so that they are explainable and defendable to many different groups of people.

Once we all agree on a motivation and goals for our science, it is more straightforward to collaborate and share data. It is counter-productive when people don't share their data when there is never enough and there are more than enough science questions to go around. It is also heartbreaking when people spend too much time on questions that have few ramifications. It is important to be thoughtful when creating a sampling strategy and communication plan with the broader community.

Roadblocks in eddy parameterization Ocean models are very important for understanding climate change and are an important part of climate models. Climate models are also often too grainy or coarse to represent all the processes we need, like ocean eddies, similar to an old television. Thus, eddy parameterization - or the representation of ocean eddies in a coarse simulation - is really important for present and future modeling efforts because of the importance of eddies in setting the global mean climate state. We seem to have not made much progress in improving how models represent these sub-grid scale processes. While some classes of models now resolve eddies, there are many that do not, including long term paleoclimate models that will still need parameterizations into the distant future. I believe 
that new numerical techniques derived from artificial intelligence and machine learning could really improve the parameterizations. I am excited by the recent proposals that have been made to explore different new options for parameterizations.

Poor communication amongst scientists Communication and teaching are vital skills for every scientist. If we cannot communicate our results, then it is like we never did the study. More than half of the talks that I go to are terrible and incomprehensible to even people in the same discipline. Papers are also poorly written, too dense, and fail to present the results for non-experts. It is really important to communicate well with our colleagues outside our fields so that we can work together to improve the state of science, learn from each other's ideas, and grow together. Many of the communication problems can be solved by keeping the audience in mind, which generally contains at least one beginning graduate student. If it is not simple enough to explain to a student, I believe that the idea still needs refining.

\subsubsection{Research Tools to Develop}

Global Array of Biogeochemical Argo floats Ordinary Argo floats revolutionized what we know about the global heat budget by measuring the heat uptake by the ocean, including the anthropogenic heat uptake by the ocean Stocker et al. 2013. This has been fundamental for constraining global climate models and understanding how the global system will change with climate change. Similarly, we still don't have a good handle on the global oceanic carbon dioxide budget. Biogeochemical Argo floats have been piloted in the Southern Ocean with great success and have provided the first seasonal variability estimates of air-sea carbon fluxes Gray et al. 2018. I believe that it is time to introduce a global biogeochemical float array so that we can constrain the ocean's role in the global carbon budget. This has already been proposed to NSF, so I am hopeful that it will get funded soon. On top of instrument networks, we also need models that incorporate the data to provide a near-real-time estimate of ocean circulation.

Global ocean state estimate in near real time with high resolution The introduction of atmospheric reanalysis data revolutionized meteorology by allowing for new kinds of scientific studies and by filling in gaps in data and understanding with near-real time data. I believe a similar tool for the ocean would revolutionize a similar amount. Developing such a tool would be challenging, in part because the ocean has much smaller length scales than atmospheric dynamics, so much more computing power is required and not enough ocean observations. 
There have been attempts to make this happen, primarily the ECCO state estimate, and an higher resolution version of ECCO in the Southern Ocean called SOSE. These products lack important dynamics because of resolution issues, not enough data to constrain the estimates, and too short a time scale to address anything more than seasonal variability. An improved state estimate would change how we do science in the ocean. On top of this, we need to develop other additional techniques for analysis, sampling, and modeling.

Developing new techniques for analysis, sampling, modeling We have a lot to learn about the ocean-earth-atmosphere-cryosphere system and how it interacts with climate. However, it seems like we don't have enough tools (or human-power) for the job. I believe we need to start incorporating machine learning and other new analytical techniques to analyze the model output that we have. Also, we need to improve climate models and other numerical models to more quickly and accurately represent atmospheric and oceanic processes. On top of that, new instruments and sensors that allow cheaper, better measurements are necessary for the global state estimate and understanding the climate system.

Because of this need, I have been interested in developing a new analytical technique that incorporates knowledge from applied mathematics to analyze the turbulence. This technique isolates the role of the turbulence in setting the mean state of the Southern Ocean. I plan on applying this technique to understand how the Southern Ocean dynamics and ocean biogeochemistry lead to changes in climate. We need new techniques like this so we can address the incredible challenge of climate change. 


\section{Appendix A}

\section{Model Configuration for Chapter 2}

\section{Model configuration.}

For numerical experiments we use the MITgcm model in a $2^{\circ}$ horizontal resolution configuration with a northern basin and a re-entrant channel in the south shown in Fig. 2 Marshall et al. 1997. We use 40 vertical layers ranging from $37 \mathrm{~m}$ thick at the surface to $159 \mathrm{~m}$ thick at the bottom and a linear equation of state with the same $\alpha$ and $\beta$ values as the analytical model. At the bottom we use a free-slip boundary condition and a no-slip condition along the side walls. The MITgcm 'seaice' package is used to produce the required buoyancy flux over the channel Jansen and Nadeau 2016. We cannot use a simple restoring boundary condition of the surface ocean temperature to a prescribed atmospheric temperature, because of the presence of sea ice. We instead follow the approach described in Jansen 2017] where the sensible heat fluxes are calculated using bulk formulae, and an additional radiative flux is prescribed as $F_{r}=\sigma\left(T_{s}^{4}-T_{a}^{4}\right)$ where $T_{s}$ is the ocean or ice surface temperature and $T_{a}$ is the prescribed atmospheric temperature, acting as a relaxation for the surface of the ocean or ice.

We prescribe the freshwater flux as evaporation minus precipitation (E-P):

$$
E-P=E_{0} f(y)
$$

where $f(y)$ is a function of latitude with units of $\mathrm{m} \mathrm{s}^{-1}$ and a magnitude corresponding to present day freshwater fluxes as shown in Figure 2-2 b. The function $f(y)$ stays the same in all simulations while we vary the magnitude of the freshwater flux through $E_{0}$. We apply 
a zonal wind stress only over the latitudes of the southern channel, which is imposed as an atmospheric zonal wind from which the EXF package Large and Pond, 2002] computes the surface stress (Figure 2-2c).

A Gent-McWilliams closure is used to parameterize the baroclinic eddies, which is crucial to get a reasonable stratification in the southern channel [Gent and McWilliams, 1990]. The Gent-McWilliams coefficient and isopycnal diffusivity are both set to be $1000 \mathrm{~m}^{2} \mathrm{~s}^{-1}$. The vertical diffusivity is set to have large values $\left(10^{-2} \mathrm{~m} \mathrm{~s}^{-2}\right)$ near the surface to represent the strong mixing generated by air-sea fluxes over a $200 \mathrm{~m}$ deep mixed layer, then rapidly decreases in the interior to reach its minimum of $1.4 \times 10^{-5} \mathrm{~m} \mathrm{~s}^{-2}$ at around $200 \mathrm{~m}$ and then increases gradually with depth to a bottom value of $2 \times 10^{-4} \mathrm{~m} \mathrm{~s}^{-2}$ Wolfe and Cessi, 2015. The mixed layer depth value is chosen to be representative of high latitudes where deep convection, the focus of this study, occurs. 


\section{Appendix B}

\section{ACC Meanders, Energy Transfer and Mixed Barotropic-Baroclinic \\ Instability}

(C)American Meteorological Society. Use with permission. ${ }^{1}$

\section{B.1 Introduction}

The Antarctic Circumpolar Current (ACC) plays a key role in the ventilation of dense water masses and the closure of the global overturning circulation [e.g. Marshall and Speer, 2012]. Recent work has highlighted the impact of the ACC's local dynamics on inter-basin transport Jones and Cessi, 2016; Thompson et al. 2016] as well as meridional heat and tracer transport Dufour et al., 2015. Although eddy kinetic energy (EKE) and isopycnal slopes vary along the path of the ACC, zonally-averaged isopycnal slopes have, in general, been found to be insensitive to changes in the wind stress in models and observations Böning et al. 2008, Abernathey et al., 2011. This effect and the relative insensitivity of the ACC's zonal baroclinic transport to an observed increase in wind stress over the ACC between 1958 and 2000 Marshall and Radko, 2003, commonly referred to as "eddy saturation" Hogg et al., 2008, 2015a, has highlighted the need to mechanistically understand both the global and local response of the ACC to a modified surface wind stress [see also Downes and Hogg, 2013,

1 Youngs et al. 2017 
Bishop et al. 2016]. There is evidence that zonally-symmetric increases in wind stress are principally balanced by localized changes in bottom form drag, communicated via interfacial form stress Ward and Hogg, 2011, Thompson and Naveira Garabato, 2014, Masich et al., 2015 .

Intensification of the surface wind stress over the ACC has been linked to an increase in the baroclinicity of the flow and a subsequent release of energy through baroclinic instability, diagnosed from increased EKE calculated from satellite altimetry-derived velocities Meredith and Hogg, 2006, Hogg et al., 2015a. Locally, the increase in EKE is dominated by regions immediately downstream of major topographic features; in regions removed from topography, changes are more moderate. These regions may even experience a suppression of EKE Bischoff and Thompson, 2014, Abernathey and Cessi, 2014, Bishop et al., 2016]. The magnitude of tracer transport is also heterogeneously distributed in the ACC, with modulations set by interactions between the flow and topographic features Lu and Speer, 2010 , Ferrari and Nikurashin, 2010, Naveira Garabato et al., 2011; Thompson and Sallée, 2012, Abernathey and Cessi, 2014, Dufour et al., 2015. Most studies have sought to understand this spatial variability in terms of modifications to the flow's baroclinicity Chapman et al. 2015, Bischoff and Thompson, 2014], although interactions with topography also produce significant changes to the flow's barotropic shear. Here we focus on along-stream transitions in the dynamical regimes of an idealized ACC meander, accounting for both eddy buoyancy and momentum fluxes and thus both baroclinic and barotropic instability, respectively.

Our analysis will primarily consider the dynamical properties of the major standing meanders of the ACC. These regions, located downstream of topographic features, share many similarities with atmospheric storm tracks - regions of elevated EKE Hoskins and Valdes, 1990, Hoskins and Hodges, 2002; Chang et al., 2002]. The shape and life cycle of atmospheric storm tracks have been, in part, linked to the standing components (i.e. standing meanders), and their ability to modify the local baroclinicity of the flow Kaspi and Schneider, 2011, 2013]. While baroclinic instability plays a key role in these interactions, in the atmosphere barotropic instabilities also influence standing meanders Williams et al. 2007. Chang et al. 2002. A defining characteristic of atmospheric storm tracks is a displacement of regions of peak baroclinicity and peak EKE, which also occurs in fully non-linear ACClike scenarios Chapman et al. 2015 and is related to classical studies of linear instability in domains with along-stream variations in the background stratification Pierrehumbert, 1984, 
Pedlosky, 1989. A key difference is that oceanic standing meanders, or storm tracks, have a separation of characteristic length scales between the standing meander and transient eddies Williams et al., 2007; Chapman et al., 2015, which influence energy transfer properties of the flow.

An example of the interplay between barotropic and baroclinic instabilities is the barotropic governor. This mechanism was first introduced by James and Gray [1986] to explain why atmospheric simulations with the same baroclinicity but larger barotropic shears have smaller eddy kinetic energy. James 1987 presented both linear and non-linear characteristics associated with the barotropic governor and found that barotropic shear may suppress the growth of a baroclinically-unstable fluid due to the destructive effect of the shear on the exponentially-growing normal modes. James 1987] also recognized that the decay of baroclinic eddies may also be responsible for generating or sustaining the barotropic shear. Building on the non-linear aspect of the barotropic governor, Nakamura 1993 specifically showed that normal-mode baroclinic waves are sensitive to vertically-integrated eddy momentum fluxes generated by barotropic shear. The resulting up-gradient momentum fluxes limit the conversion of available potential energy into eddy kinetic energy. In other ocean-focused studies, Rivière et al. 2004 found that the baroclinicity often overwhelms the barotropic governor mechanism, but Klocker et al. 2016 and Solodoch et al. 2016 presented Southern Ocean and idealized (respectively) flow regimes with characteristics consistent with the barotropic governor. In this study, by diagnosing both momentum and buoyancy fluxes within and far from the meander, we examine the relevance of this mechanism in the ACC.

Here we adopt the geometric stability method, described in Marshall et al. 2012, Waterman and Hoskins 2013, and Waterman and Lilly 2015 to examine the stability properties and energy conversion rates of an ACC meander with a higher degree of granularity than in earlier studies that have focused primarily on differences between meander and quasizonal regions of the ACC. Waterman and Hoskins 2013 showed how the statistics of eddy geometry, calculated for an idealized barotropic jet, agree with simple predictions of eddymean energy exchange. More recently, Waterman and Lilly 2015 used the same model to consider the geometric decomposition of eddy feedbacks and showed that both the angle and the anisotropy of transient eddies are important for the evolution of the mean flow. Recently, Tamarin et al. 2016] extended this horizontal analysis to show the consistency of ray-tracing theory with the geometric method. This method is useful for its physical 
interpretation and connection to linear stability theory. A motivation for this approach is that the horizontal eddy shape can be observed remotely, leading to an assessment of eddy momentum fluxes without the need to compute correlations Marshall et al., 2012. A global analysis in this framework was carried out by Stewart et al. 2015 using a global general circulation model. These previous studies (with the exception of Marshall et al. [2012]) only consider barotropic flow regimes, and therefore the statistical description of the eddy geometry is limited to the horizontal plane. The ACC, however, has a significant baroclinic component, which motivates our adoption of the generalized three-dimensional eddy geometry developed by Marshall et al. 2012.

Using an idealized MITgcm channel model with a meridional ridge, we examine the threedimensional shape of transient eddies to determine their evolution and interaction with ACC meanders; these diagnostics are compared with energy conversion terms. Diagnosing both kinetic and potential energy conversions allows us to assess the relative importance of both barotropic and baroclinic instabilities in the life cycle of an ACC meander. The model and methods are described in section 2. In section 3, we discuss the spatial distribution of energy conversion terms and analyze the localization of this exchange. Following Marshall et al. [2012], we examine the geometric stability of the domain in section 4. In section 5, these results are interpreted in terms of the interaction between barotropic and baroclinic instabilities and are compared to previous studies of storm tracks and jet regions; we conclude with a summary in section 6.

\section{B.2 Methods}

\section{B.2.1 Energy Conversion}

We begin our analysis of the meander system by considering both the kinetic and potential energy budgets. We focus on those terms that describe the exchange of energy between mean and eddy energy reservoirs. We define the mean kinetic energy (MKE) of the flow as

$$
M K E(x, y, z)=0.5 \rho_{0}\left(\bar{u}^{2}+\bar{v}^{2}\right),
$$


and the eddy kinetic energy (EKE) as

$$
E K E(x, y, z)=0.5 \rho_{0} \overline{\left(u^{\prime 2}+v^{\prime 2}\right)}
$$

where $u$ is the zonal velocity, $v$ is the meridional velocity, $\rho_{0}$ is a constant reference density, overbars denote a time average (in our simulations a period of 13 years), and primes denote deviations from the time mean. For the potential energy, we adopt the quasi-geostrophic framework. This implies an assumption that perturbations to the stratification are small compared to the mean stratification. This could appropriately be questioned across the standing meander and downstream regions, but we adopt this assumption for tractability and note that the quasi-geostrophic framework has been shown to often apply outside its formal limits Williams et al. 2010]. The mean available potential energy (MAPE) is given by

$$
M A P E(x, y, z)=\frac{0.5}{\mathcal{N}_{0}^{2}(z) \rho_{0}}{\overline{\rho^{*}(x, y, z, t)}}^{2}
$$

and the eddy available potential energy (EAPE) is given by

$$
E A P E(x, y, z)=\frac{0.5}{\mathcal{N}_{0}^{2}(z) \rho_{0}} \overline{\rho^{\prime}(x, y, z, t)^{2}},
$$

where $\mathcal{N}_{0}^{2}(z)$ is the squared buoyancy frequency computed from a domain-averaged, timemean density profile, $\rho^{*}(x, y, z, t)=\rho(x, y, z, t)-\overline{\langle\rho(x, y, z, t)\rangle}$ and $\rho^{\prime}(x, y, z, t)=\rho(x, y, z, t)-$ $\overline{\rho(x, y, z, t)}$ where $\langle\cdot\rangle$ indicate a domain average at a given depth. From these definitions, governing equations for kinetic and potential energy may be derived as in Chen et al. 2014; interactions between eddies and the mean flow give rise to exchange between these energy reservoirs. This framework also isolates the baroclinic and barotropic instability pathways, which allows comparison to the geometric method, outlined in section B.2B.2.2.

We consider the following five terms in this study following Chen et al. 2014:

- the rate of change of MAPE from horizontal eddy density (buoyancy) fluxes $\left(D_{P_{M}}\right)$

$$
D_{P_{M}}=-\frac{\overline{\rho^{*}}}{\mathcal{N}_{0}^{2}(z) \rho_{0}} \nabla_{H} \cdot \overline{\left(\mathbf{u}_{H}^{\prime} \rho^{\prime}\right)}
$$

where $\mathbf{u}_{H}$ is the horizontal velocity vector and $\nabla_{H}$ is the horizontal gradient operator;

- the rate of change of eddy energy (EKE and EAPE) due to horizontal eddy density 
(buoyancy) fluxes $\left(D_{P_{E}}\right)$

$$
D_{P_{E}}=-\frac{1}{\mathcal{N}_{0}^{2}(z) \rho_{0}} \overline{\left(\mathbf{u}_{H}^{\prime} \rho^{\prime}\right)} \cdot \nabla_{H} \overline{\rho^{*}}
$$

- the rate of change in EKE from $\operatorname{EAPE}\left(D_{K_{E}}\right)$

$$
D_{K_{E}}=-g \overline{\rho^{\prime} w^{\prime}}
$$

where $w$ is the vertical velocity and $g$ is the gravitational acceleration constant;

- the rate of change in MKE due to eddy momentum fluxes $\left(M_{K_{M}}\right)$

$$
M_{K_{M}}=-\rho_{0}\left[\bar{u} \nabla \cdot \overline{\left(u^{\prime} \mathbf{u}^{\prime}\right)}+\bar{v} \nabla \cdot \overline{\left(v^{\prime} \mathbf{u}^{\prime}\right)}\right]
$$

- the rate of change of EKE due to eddy momentum fluxes $\left(M_{K_{E}}\right)$

$$
M_{K_{E}}=-\rho_{0}\left[\overline{u^{\prime} \mathbf{u}^{\prime}} \cdot \nabla \bar{u}+\overline{v^{\prime} \mathbf{u}^{\prime}} \cdot \nabla \bar{v}\right] .
$$

The baroclinic instability pathway is described by the $D$-terms, $D_{P_{M}}, D_{P_{E}}$ and $D_{K_{E}}$, as they represent the flow of energy from MAPE to EAPE to EKE due to eddies. Barotropic instability is represented by the $M$-terms, $M_{K_{M}}$ and $M_{K_{E}}$ because these terms represent the extraction of MKE due to eddies and the growth of EKE, respectively. $D_{P_{M}}$ and $D_{P_{E}}$ are related but not identical: integrating $D_{P_{M}}$ by parts results in $D_{P_{E}}$ as well as an additional term that integrates to zero over the domain but does not disappear locally (the same is true when the two terms are swapped). The additional term here contains the energy advection and other residual terms. The relationship between $M_{K_{M}}$ and $M_{K_{E}}$ is analogous. The other terms in the energy equations, such as dissipation, are not pertinent for our stability analysis and are not discussed. See Chen et al. 2014 for further information about these terms.

We note that $D_{P_{E}}$ contains both the rotational and divergent components of the buoyancy fluxes. The rotational component, which is customarily removed, corresponds to the growth and decay of eddies in the domain [Marshall and Shutts, 1981]. Since we are interested in the eddy life cycles, we choose to use the full fluxes. 


\section{B.2.2 Eddy Geometry}

In addition to the energy conversion terms described above, we also employ an eddy shape analysis to examine eddy-mean flow interactions within the meander. While the geometric diagnostics essentially use the same information as the conversion terms - eddy fluxes of momentum and buoyancy - the geometric framework has proven insightful in mapping the spatial distribution of eddy-mean flow interaction Waterman and Lilly, 2015]. The derivation of the eddy geometry terms is provided in Marshall et al. 2012].

We define

$$
M=\frac{\overline{v^{\prime 2}-u^{\prime 2}}}{2}, \quad N=\overline{u^{\prime} v^{\prime}}
$$

which are related to the eddy Reynolds stresses, and

$$
R=\frac{f_{0} g}{\mathcal{N}_{0}^{2}(z) \rho_{0}} \overline{u^{\prime} \rho^{\prime}}, \quad S=\frac{f_{0} g}{\mathcal{N}_{0}^{2}(z) \rho_{0}} \overline{v^{\prime} \rho^{\prime}}
$$

which are components of eddy buoyancy flux and are proportional to eddy interfacial form stress.

From these terms we define a horizontal anisotropy parameter

$$
\gamma_{m}=\frac{\sqrt{M^{2}+N^{2}}}{E K E}, \quad 0 \leq \gamma_{m} \leq 1
$$

which is the ratio of the eddy Reynolds stresses to the eddy kinetic energy. This measures the eccentricity in the horizontal shape of the eddy, or equivalently the anisotropy in the eddy momentum fluxes. When $\gamma_{m} \approx 0$ the flow is approximately isotropic; the flow becomes more anisotropic as $\gamma_{m}$ approaches 1. A measure of the dominant (or time-averaged) angle $\phi_{m}$ of the eddy momentum fluxes is given by:

$$
\phi_{m}=\frac{1}{2} \cos ^{-1}\left(-\frac{M}{\sqrt{M^{2}+N^{2}}}\right), \quad 0 \leq \phi_{m} \leq \pi
$$

Here, $\phi_{m}$ is the angle of the eddy momentum fluxes with respect to the zonal direction in the horizontal plane (Figure B-1). This decomposition can also be undertaken for the buoyancy fluxes, with

$$
\gamma_{b}=\frac{N(z)}{2 f_{0}} \sqrt{\frac{R^{2}+S^{2}}{E K E * E A P E}}, \quad 0 \leq \gamma_{b} \leq 1
$$

which represents the relative magnitude of the eddy buoyancy fluxes to the eddy poten- 
tial and kinetic energies, or the anisotropy in the eddy buoyancy fluxes. There is also a corresponding angle

$$
\phi_{b}=\cos ^{-1}\left(\frac{R}{\sqrt{R^{2}+S^{2}}}\right), \quad-\pi \leq \phi_{b} \leq \pi
$$

which gives the dominant direction of the eddy buoyancy fluxes in the horizontal plane. We also define

$$
\frac{E A P E}{E K E}=\tan ^{2} \lambda, \quad 0 \leq \lambda \leq \pi / 2
$$

where $\lambda$ measures the partitioning of total eddy energy into kinetic and potential components, or the baroclinicity of the flow. We then project the eddy buoyancy fluxes into the vertical-along stream plane to describe the eddy fluxes for baroclinic eddies similar to the horizontal interpretation used for barotropic eddies. We take

$$
\tan 2 \phi_{t}=\gamma_{b} \tan 2 \lambda, \quad \gamma_{t}=\frac{\cos 2 \lambda}{\cos 2 \phi_{t}}, \quad 0 \leq \gamma_{t} \leq 1
$$

where $\phi_{t}$ is the angle in the $\mathrm{x}-\mathrm{z}$ plane with respect to the horizontal plane, and $\gamma_{t}$ is a measure of the eccentricity of the eddy fluxes in the vertical direction (Figure B-1). Since the channel flow is not purely zonal, we project $\phi_{t}$ onto the plane that is perpendicular to the direction of $\phi_{b}$, giving the sign of the eddy buoyancy fluxes. These diagnostics provide the stability and anisotropy parameters for baroclinic instability, which is related to the magnitude and direction of the eddy buoyancy fluxes Marshall et al., 2012.

Larger anisotropy - given by $\gamma_{m}$ in the horizontal and $\gamma_{t}$ in the vertical - of the eddy fluxes is associated with stronger eddy-mean flow interactions. The angle of the eddy $\left(\phi_{m}, \phi_{t}\right)$, with respect to the mean flow determines the stability of the flow, represented schematically in Figure B-1. When the eddy, represented by an eddy stream function, tilts against the mean flow shear, the perturbation is growing and extracting energy from the mean flow. When the eddy is tilted with the mean flow shear, the perturbation is decaying and exchanging energy back into the mean flow Pedlosky, 2013. For energy to transfer between the mean and eddy components, the eddy flux anisotropy and eddy stream function tilt must both be non-zero, with larger anisotropy, $\gamma \rightarrow 1$, providing more effective energy transfer. This conceptual picture has been effective in describing barotropic flows Waterman and Lilly, 2015], and can be extended to describe the physical shape of a barotropic eddy. The physical 
interpretation is more challenging when extended to the vertical because the eddy geometry now depends on eddy buoyancy fluxes instead of just eddy momentum fluxes, i.e. depends on $u^{\prime}, v^{\prime}$ and $b^{\prime}$. Nevertheless, below we show that the eddy orientation provides a consistent picture when compared to the more traditional energy conversion terms.

\section{B.2.3 Model}

The goal of our model configuration is to resolve mesoscale eddy dynamics in a re-entrant channel of a sufficient length that will allow us to analyze the along-stream structure of the eddy-mean flow interactions in three different regimes: upstream, within and downstream of the meander. Our experiment is designed to have a negligible residual overturning circulation as there is no surface buoyancy forcing and weak interior diapycnal mixing. Thus, the eddy overturning completely balances the wind-induced circulation. We use the MIT$\mathrm{gcm}$ Marshall et al. 1997 in the Boussinesq and hydrostatic approximations on a $\beta$-plane $\left(f(y)=-10^{-4}+10^{-11} \cdot y\right)$ with a Cartesian grid where $x$ is the zonal coordinate and $y$ is the meridional coordinate. The domain is $6000 \mathrm{~km}$ long (zonal), $2000 \mathrm{~km}$ wide (meridional), and $4000 \mathrm{~m}$ deep (vertical). We add topography in the form of a simple cross channel Gaussian ridge centered at $x=x_{0}$ :

$$
h_{b}(x)=-H+h_{0} e^{-\left(x-x_{0}\right)^{2} / \sigma^{2}}
$$

where $h_{0}=1000 \mathrm{~m}, \sigma=200 \mathrm{~km}$ and $x_{0}=800 \mathrm{~km}$ (Fig. B-2). The blocking ridge allows the momentum input by the wind to be dissipated by bottom form drag. The deformation radius is approximately $35 \mathrm{~km}$ and the grid spacing is $10 \mathrm{~km}$, so that the model is eddyresolving. There are 32 vertical levels increasing from $50 \mathrm{~m}$ at the surface to $200 \mathrm{~m}$ at depth. Linear bottom drag with a drag coefficient of $1.1 \cdot 10^{-3} \mathrm{~m} \mathrm{~s}^{-1}$ is applied and we impose free slip boundary conditions at the north and south boundaries. The density is prescribed by a linear equation of state with temperature only; the reference density is $\rho_{0}=1000 \mathrm{~kg} \mathrm{~m}^{-3}$ and the thermal expansion coefficient is $\alpha=2 \cdot 10^{-4}{ }^{\circ} C^{-1}$. The model horizontal explicit diffusivity is set to zero, and the vertical is $5 \cdot 10^{-6} \mathrm{~m}^{2} \mathrm{~s}^{-1}$, which is comparable to the effective numerical diffusivity, meaning the interior is almost completely adiabatic. Sub-grid processes are represented by a horizontal eddy viscosity of $12 \mathrm{~m}^{2} \mathrm{~s}^{-1}$ and a vertical viscosity of $3 \cdot 10^{-4} \mathrm{~m}^{2} \mathrm{~s}^{-1}$. The temperature at the northern boundary is relaxed to an exponential 
profile from $20^{\circ} \mathrm{C}$ at the surface to $0^{\circ} \mathrm{C}$ at depth:

$$
\theta(z)=20\left(e^{z / 1000}-e^{-H / 1000}\right) /\left(1-e^{-H / 1000}\right)
$$

with a relaxation period of one week. This relaxation is applied linearly over a meridional extent of $100 \mathrm{~km}$. We apply a zonal wind stress , $\tau^{x}(y)=\tau_{0} \sin \left(\pi y / L_{y}\right)$, with two different maxima, $\tau_{0}=0.1$ and $0.2 \mathrm{Nm}^{-2}$, and no surface buoyancy forcing. We use yearly averages of 13 model years after an equilibration period of 27 years for both wind stresses. Examples of the model output are shown in Fig. B-2, The depth and spatially-averaged zonal velocity is $4 \mathrm{~cm} / \mathrm{s}$ for $\tau_{0}=0.1 \mathrm{~N} \mathrm{~m}^{-2}$ and $4.4 \mathrm{~cm} \mathrm{~s}^{-1}$ for $\tau_{0}=0.2 \mathrm{~N} \mathrm{~m}^{-2}$. The spatial-averaged surface zonal velocities are $13 \mathrm{~cm} \mathrm{~s}^{-1}$ for $\tau_{0}=0.1 \mathrm{~N} \mathrm{~m}^{-2}$ and $13.4 \mathrm{~cm} \mathrm{~s}^{-1}$ for $\tau_{0}=0.2 \mathrm{~N} \mathrm{~m}^{-2}$. Mean surface velocities of the ACC are around $20 \mathrm{~cm} \mathrm{~s}^{-1}$ Talley, 2011.

\section{B.3 Energy Conversion}

We first present the energy conversion terms to diagnose the spatial patterns of the eddymean flow interactions. The diagnosed depth-averaged eddy energy conversion terms are defined in section B.2[B.2.1. The largest amplitudes of $D_{P_{M}}(\mathrm{B.5})$ are negative, indicating a loss of MAPE and occur on the flanks of the jet, whereas the largest amplitudes of $D_{P_{E}}(\mathrm{~B} .6)$ are positive, indicating a gain of EAPE and occur within the core of the jet (Figs. B-3 B-4). This implies that the eddy-mean flow interactions are spatially non-local. Both $D_{P_{M}}$ and $D_{P_{E}}$ are largest in the lee of ridge, but are peaked upstream of the region where EKE is largest (Fig. B-2).

In contrast, the patterns of $D_{P_{E}}$ and $D_{K_{E}}$ are quite similar and have the same sign. This is consistent with the baroclinic instability pathway, highlighting regions where EAPE is converted into EKE. The magnitude of $D_{K_{E}}(\mathrm{~B} .7)$ is smaller than $D_{P_{E}}$, suggesting that not all of the EAPE is transformed into EKE in these locations. This difference results from a combination of a flux of EAPE out of the domain, as well as dissipation, and potentially conversion into MKE Chen et al., 2016.

The conversion of MKE from the eddy momentum fluxes, $M_{K_{M}}(\mathrm{~B} .8)$, is peaked at the same location as the MKE field (not shown), within the core of the jet, and is a sink of MKE. $M_{K_{E}}$ (B.9) has its largest amplitude further downstream along the flanks of the jet and coincides with the region of largest horizontal shear. Here the Reynolds stresses are 
most efficient at extracting energy from the mean flow, resulting in barotropic instability. Overall, the conversion from EAPE $\left(D_{K_{E}}\right)$ and from MKE $\left(M_{K_{E}}\right)$ into EKE have the same magnitudes in the meander region both peaking around $0.1 \mathrm{Wm}^{-2}$, consistent with a mixed instability process.

Comparing the two imposed wind stress amplitudes, all conversion terms have larger magnitudes for the $\tau_{0}=0.2 \mathrm{~N} \mathrm{~m}^{-2}$ experiment (Figs. B-3 and B-4). This is consistent with a mechanically-induced increase in the APE reservoir; the stronger wind stress generates a larger amplitude near-surface Ekman convergence and divergence. However, qualitatively there is no appreciable change in the physical extent of the conversion region with a change in winds, which differs from the results of Bischoff and Thompson [2014. In this previous study, which used a Gaussian bump instead of a ridge, the physical extent of the storm track was strongly sensitive to the magnitude of the wind stress.

The relative insensitivity of the zonal baroclinic mean flow to the wind stress is a common feature of these ACC-like channel models, a property known as eddy saturation, but the sensitivity of the energy budget and the exchange between different reservoirs, has been explored to a lesser extent. We consider the regionally-averaged energy characteristics using a Lorenz diagram similar to those used in Chen et al. 2014] (Fig. B-5). Within the meander, defined by boxes on the left-hand side of Figs. B-3 and B-4, eddy fluxes consistent with barotropic instability are a primary component of energy transfer. This is represented by the conversion of MKE into EKE, which occurs in a barotropically unstable system. In contrast, downstream of the meander, defined by the right-hand boxes in Figs. B-3 and B-4. EKE is converted back to MKE, consistent with up-gradient eddy momentum fluxes. In the downstream domain, there is an excess of energy being introduced from upstream, consistent with advection out of the upstream domain shown in the Lorenz diagram.

For the potential energy transfer components of the Lorenz diagram, in both regions, MAPE is converted into EAPE, which is then converted into EKE: the traditional baroclinic instability energy pathway. However, $D_{P_{E}}$ is larger than $D_{P_{M}}$, so EAPE enters each domain through a different process. This "leak" in the system could be advection downstream, a delay phenomenon discussed in Chen et al. [2016], the propagation of energy downstream at the group velocity Chapman et al. 2015, or another process. This suggests that eddy potential energy may be transported downstream where there is sufficient time to complete the conversion to EKE. This is supported by the displacement between regions of enhanced 
baroclinicity and the maximum in EKE Bischoff and Thompson, 2014, Chapman et al., 2015].

With a change in wind stress, the magnitude of the energy transfers in the Lorenz diagram tend to increase. Within the meander, the conversion of MAPE to EAPE is doubled when the wind stress is doubled, but the conversion of EAPE to EKE is only increased by $60 \%$. The conversion of MKE to EKE on the other hand increases by over 3.5 times. We see that as the wind stress increases, the total contribution to EKE changes from mostly from EAPE to almost the exact same magnitude between EAPE and MKE. In the downstream region, the conversion of EKE to MKE is doubled when the wind stress is doubled, but the conversion between MAPE to EKE is basically unchanged.

To summarize, in the meander regions, the fluxes of EAPE to EKE and of MKE to EKE are both down-gradient and roughly the same magnitude, consistent with mixed instability. In the downstream region, the buoyancy fluxes are down-gradient, consistent with baroclinic instability but the momentum fluxes are up-gradient. However, in this region the energy leaving EKE due to eddy momentum fluxes $\left(M_{K_{E}}\right)$ is significantly smaller than the energy entering EKE though the baroclinic pathway $\left(D_{K_{E}}\right)$. These up-gradient momentum fluxes could result from several phenomena, including eddies that have been generated elsewhere, advected into a region and subsequently sheared by the mean flow or through baroclinic instability Pedlosky, 2013.

\section{B.4 Geometric Analysis}

Analyzing the same simulations using the geometric framework described in section B.2B.2.2 produces a similar interpretation of the instability and energy conversion properties of the flow. However, this geometric method can provide a more physical interpretation of the energy exchange terms. The angles of the eddies with respect to the mean flow have a direct analogy to linear stability theory Marshall et al. 2012, Pedlosky, 2013. When the eddies are tilted with the shear, they are giving energy to the mean flow, corresponding to stability, and when the eddies are tilted against the shear, they are growing and taking energy from the mean flow, corresponding to instability (Fig. B-1).

For our geometric analysis we first analyze the flow in the horizontal, 2-D plane, similar to Waterman and Lilly 2015. Using depth-averaged (i.e. vertical average weighted by the layer 
thickness) anisotropy, we find that topography locally modifies eddy anisotropy (Figs. B-6 and B-7). In the case where $\tau_{0}=0.2 \mathrm{~N} \mathrm{~m}^{-2}$, elevated anisotropy occurs on the flanks of the jet as the meander deflects southward. This highlights a key role for the topography: in order to conserve potential vorticity and mass, streamlines tighten upon crossing the ridge, which increases the horizontal shear and the potential for barotropic instability. We also speculate that rapid changes in the orientation of the streamlines can produce regions where coherent eddies are transiently tilted against the mean shear before adjusting to the new shear orientation - a form of eddy memory related to the finite adjustment time of a coherent eddy in a spatially-variable mean flow. Analysis of the horizontal eddy tilt is complicated due to the changing orientation of the streamlines (as opposed to perfectly horizontal streamlines Waterman and Lilly, 2015) . Within the meander itself, though, the momentum fluxes tend to be down-gradient, or the eddies are tilted against the mean flow shear. Immediately downstream of the meander at about $x=1500 \mathrm{~km}$, where the analysis is easier, all eddies tilt with the shear corresponding to up-gradient momentum fluxes. The sign of the momentum fluxes agrees with the distribution of $M_{K_{E}}$ (Fig. B-4(d)). There are elevated regions of eddy anisotropy near the northern and southern boundaries that are due to geometrical constraints of the numerical simulation and we do not focus on these regions (Figs. B-6 and B-7).

Next we consider the vertical eddy geometry (3-D flow), which provides information about the interactions between baroclinic eddies and the mean flow. The vertical anisotropy is high in several locations, particularly over the ridge and downstream of the ridge. There are sporadic instances of high vertical anisotropy in the remainder of the domain as well (Figs. B-8 and B-9). In the downstream, quasi-zonal jet region, the rapid changes of the eddy anisotropy is partly related to an imperfect agreement between the jet cores and the streamline used for the along-stream analysis. The flow generally has down-gradient buoyancy fluxes over and around the ridge. The one exception is that for the $\tau_{0}=0.2 \mathrm{~N}$ $\mathrm{m}^{-2}$ case, just downstream of the ridge, the flow has both low anisotropy and up-gradient buoyancy fluxes in a small region.

A comparison of the spatial characteristics of the vertical eddy geometry in Figs B-8 and B-9 are also largely consistent with the $D_{P_{E}}$ (conversion to EAPE from MAPE) patterns in Figs. B-3(b) and B-4(b). For instance, for $\tau_{0}=0.2 \mathrm{~N} \mathrm{~m}^{-2}$, the flow has up-gradient buoyancy fluxes before the maximum shear, as indicated by the vertical anisotropy com- 
bined with the vertical tilt. Downstream of the maximum shear, the flow has down-gradient buoyancy fluxes, as indicated by the large conversions into $\operatorname{EAPE}\left(D_{P_{E}}\right)$, and the high vertical anisotropy and unstable tilt in the vertical. When $\tau_{0}=0.1 \mathrm{~N} \mathrm{~m}^{-2}$, the flow has down-gradient buoyancy fluxes, which is again consistent with the eddy vertical tilt and anisotropy. Eddy buoyancy fluxes are more consistently down-gradient throughout the domain, as compared to the eddy momentum fluxes, but the amplitude of the buoyancy fluxes and momentum fluxes become similar in the meander region leading to mixed instability.

\section{B.5 Discussion}

\section{B.5.1 Mixed Instability in the ACC}

The large-scale tilt of density surfaces across the ACC highlights the importance of baroclinic instability in setting the current's equilibrated structure. A close analysis of eddy energy transfers in the most energetic regions of the ACC, the major standing meanders, suggests that baroclinic conversion alone is insufficient to describe both the stratification and distribution of EKE. The magnitude of energy transfer into EKE via $M_{K_{E}}$ and $D_{K_{E}}$, the barotropic and baroclinic pathways in the meander respectively, are approximately the same (Figures B-3 B-4 and B-5). This reflects the reduction in spatial scale of the core of the ACC, which magnifies the horizontal shear until barotropic instability becomes important [Killworth, 1980]. The flow is then in a regime of mixed instability. With a change in the magnitude of the wind stress, we see a difference in the shape of the meander, with a narrower jet for the case where $\tau_{0}=0.2 \mathrm{~N} \mathrm{~m}^{-2}$, which would imply a larger contribution of barotropic instability, a hypothesis that is verified by energy conversion terms (Figure B-5). Thus changes in wind stress could change the relative importance of barotropic and baroclinic instabilities in this system. This more complicated view of baroclinic instability is potentially a problem for model parameterizations because they do not take background shear into account, which could result in an incorrect partitioning of energy.

The notion that barotropic instability may play a leading order role in the equilibration of standing meanders and therefore impact larger-scale properties of the ACC has received little attention. Indeed, Vallis 2006 (Ch. 16.6) uses a scaling analysis to suggest that the lateral momentum fluxes can be neglected in comparison to the eddy buoyancy fluxes. However, this is based on bulk properties of the ACC, which are modified significantly within 
the standing meanders, as described above. The elevation of the eddy momentum fluxes, and the importance of barotropic instability in the meander has important implications. The momentum balance is typically described as a balance between input from surface winds and the vertical transfer, via interfacial form stress, to the solid earth. It is baroclinic instability and eddy buoyancy fluxes that carry out this vertical transfer. As eddy momentum fluxes become more important, a larger proportion of this momentum can be diverged laterally or, as shown here, exchanged back to the mean flow and carried away from the regions with significant topographic features. Finally, interfacial form stress is intricately linked to the overturning circulation through the meridional transfer of mass and heat related to the coupled nature of eddy buoyancy, heat and thickness fluxes Marshall and Speer, 2012. A change in the partitioning of barotropic or baroclinic instability properties in response to a change in surface wind stress will then also impact the overturning circulation.

As discussed in the introduction, the incomplete conversion of EAPE to EKE has, in the atmospheric literature, been associated with the barotropic governor mechanism. It occurs because during the growth process of baroclinic eddies, some of the EAPE may be converted into MKE through up-gradient momentum fluxes when there is a barotropic shear. Thompson and Richards 2011; Klocker et al. 2016] have used model output to suggest that up-gradient momentum fluxes might be relevant in the ACC. However, baroclinic growth is not the only mechanism responsible for generating up-gradient momentum fluxes. Advection of energy between different regions of the domain or the barotropic decay of eddies could also result in the up-gradient momentum flux as well. The energy analysis here indicates that up-gradient fluxes of momentum are collocated with down-gradient fluxes of buoyancy. This arrangement is consistent with the description of the barotropic governor provided by Nakamura 1993 where up-gradient momentum fluxes occur in a baroclinically unstable

flow. The flow has both a barotropic and baroclinic shear, which can interact leading to mixed instability and feedbacks.

\section{B.5.2 Meander Life Cycle}

The focus on energy transfer diagnostics across a single meander provides insight into the life cycle of fluid parcels passing through this localized region, offering a more nuanced view of eddy-mean flow interactions in the complex ACC. Upstream of the meander there are up-gradient momentum fluxes that remove energy from the eddies and accelerate the 
mean flow, predominantly in narrow jets with a lateral scale comparable to the deformation radius. The jets are steered by topography upon encountering the meridional ridge. Over the ridge, conservation of potential vorticity results in strengthening of the horizontal shear and baroclinicity of the jet(s). At the crest of the ridge, both the eddy momentum and the eddy buoyancy fluxes are transiently up-gradient. We hypothesize that the up-gradient buoyancy fluxes, in particular, are a result of an abrupt transition in the orientation of the mean flow.

Immediately downstream of the ridge, the enhancement of barotropic shear becomes a source of EKE, but may also act to suppress linear baroclinic instability through the distortion of normal modes. The displacement of the locations of maximum baroclinicity and maximum EKE has been linked to convective instability Pierrehumbert, 1984, Abernathey and Cessi, 2014], which certainly contributes to the equilibrated distribution of EKE. However, the strongest release of EKE through baroclinic instability, diagnosed by $D_{K_{E}}$, is collocated with the release of EKE through barotropic instability, diagnosed by $M_{K_{E}}$, of a similar magnitude. This is indicative of the meander region being controlled by mixed barotropic-baroclinic instability.

Based on surface velocities retrieved from altimetric observations, restricting the analysis to barotropic eddy-mean flow interactions and to relatively coarse scales $(\sim 100 \mathrm{~km})$, Williams et al. [2007] find that upstream of the maximum jet velocity the eddy vorticity fluxes are up-gradient, while downstream of this region they are down-gradient. This agrees well with the eddy momentum flux distributions seen in the barotropic component of our flow. The addition of a ridge also generates an along-stream evolution of the eddy-mean flow interactions that has a number of similarities to the idealized western boundary current jet studied by Waterman and Jayne 2011, for example high horizontal eddy anisotropy on the flanks of the jet (Fig. B-7]. Abernathey and Cessi 2014 find that eddies generated in the meander are often advected downstream, where they continue to extract energy from the mean flow, which agrees with our understanding of the energy budget in our domain showing that some processes are non-local (Figure B-5). In agreement with our analysis of standing meanders, Bischoff and Thompson [2014] find that in the lee of topography isopycnal tilt increases due to the standing component of lateral buoyancy fluxes, but is relaxed due to the transient eddy buoyancy fluxes. Complementing these earlier studies, this analysis combines both baroclinic and barotropic instabilities and their impact on the 
mean flow, as summarized in Fig. B-10.

\section{B.6 Conclusions}

This study provides one of the first applications of the geometric stability method, derived by Marshall et al. 2012, to consider both barotropic and baroclinic components of the flow. The eddy-mean flow interactions implied from this analysis are consistent with energy conversion and regional Lorenz energy diagrams diagnosed in the simulations. The consideration of both barotropic and baroclinic instability characteristics reveals the importance of barotropic instability in the ACC.

The stability analysis of the ACC meander shows the detailed evolution of the flow's stability properties through the meander, which is summarized by Fig. B-10. The region upstream of the meander is characterized by up-gradient momentum fluxes and down-gradient buoyancy fluxes, consistent with baroclinic instability and the 'barotropic governor'. In the meander itself, we have both down-gradient buoyancy and momentum fluxes consistent with both barotropic and baroclinic instability or mixed instability. Downstream of the meander, we have up-gradient momentum fluxes and down-gradient buoyancy fluxes indicating continued baroclinic growth and barotropic decay.

Simulations run with two different wind stress magnitudes resulted in qualitatively similar patterns of eddy fluxes. However, as the forcing changed, the relative interactions between the baroclinic and barotropic flows were modified. This could modify tracer transport and impact the meridional overturning circulation. Accurately representing these fluxes is likely to be critical for simulating how energy is dissipated in numerical models of the Southern Ocean. 

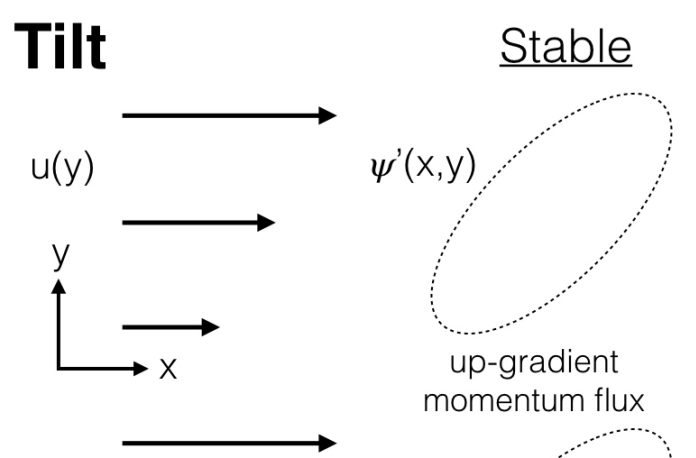

\section{Unstable}

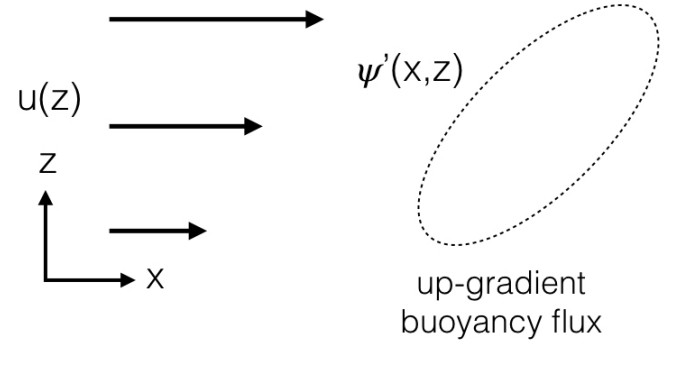

\section{Anisotropy}

Low Anisotropy

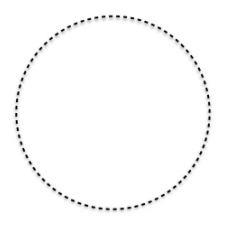

Small transfers between

eddy and mean

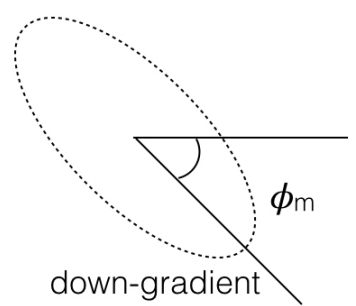

momentum flux

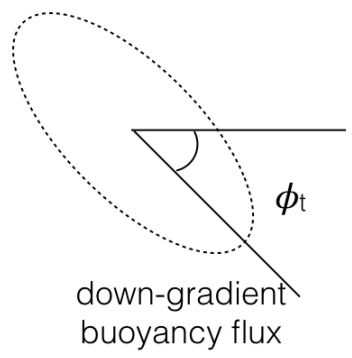

High Anisotropy

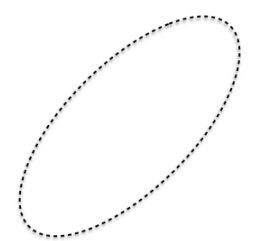

arge transfers between

eddy and mean

Figure B-1: Schematic relating eddy orientation and stability (both barotropic and baroclinic). The black arrows to the left show the shear related to the mean flow $u$, the dashed lines are the eddy stream function contours $\psi^{\prime}$. Purely barotropic eddies tilt in the horizontal (top) and $\psi^{\prime}$ is a function of $u^{\prime}$ and $v^{\prime}$; purely baroclinic eddies tilt in the vertical (middle) and $\psi^{\prime}$ is a function of $v^{\prime}$ and $b^{\prime}$. When the eddy major axis tilts with the shear, the eddies are stable and decay, giving energy to the mean flow, which increases the shear. When the eddy major axis tilts against the shear, the eddies are unstable and grow, taking energy from the mean flow, which reduces the shear. 


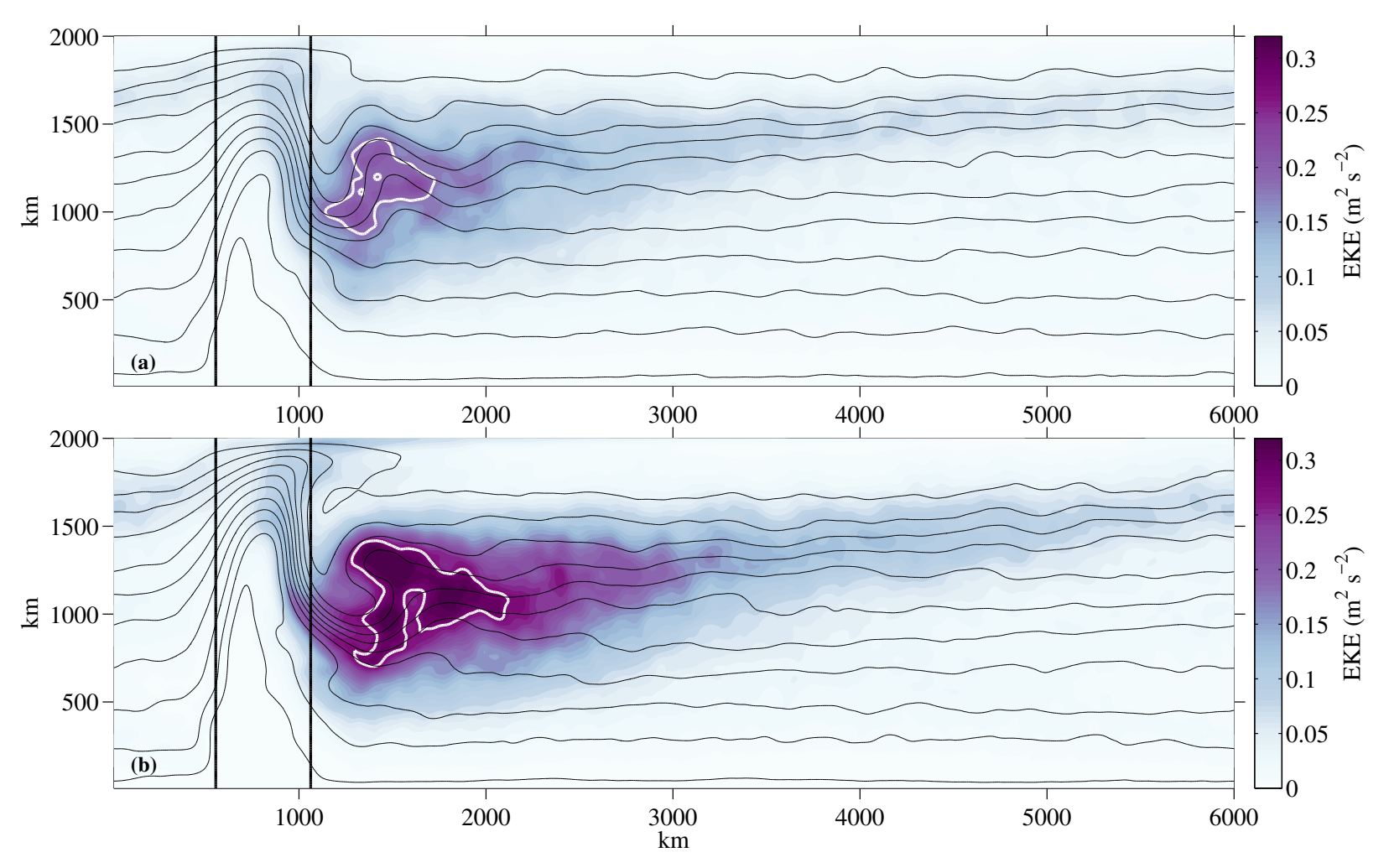

Figure B-2: Eddy kinetic energy at $177 \mathrm{~m}$ depth for experiments with wind stress forcing (a) $\tau_{0}=0.1 \mathrm{~N} \mathrm{~m}^{-2}$ and (b) $\tau=0.2 \mathrm{~N} \mathrm{~m}^{-2}$. Thick white contours indicate the region containing EKE that is at least $80 \%$ of the peak EKE. Thin lines show potential temperature contours and are separated by $1^{\circ} \mathrm{C}$. Thick lines show where the Gaussian ridge, centered at $800 \mathrm{~km}$, rises above $3800 \mathrm{~m}$ depth. 


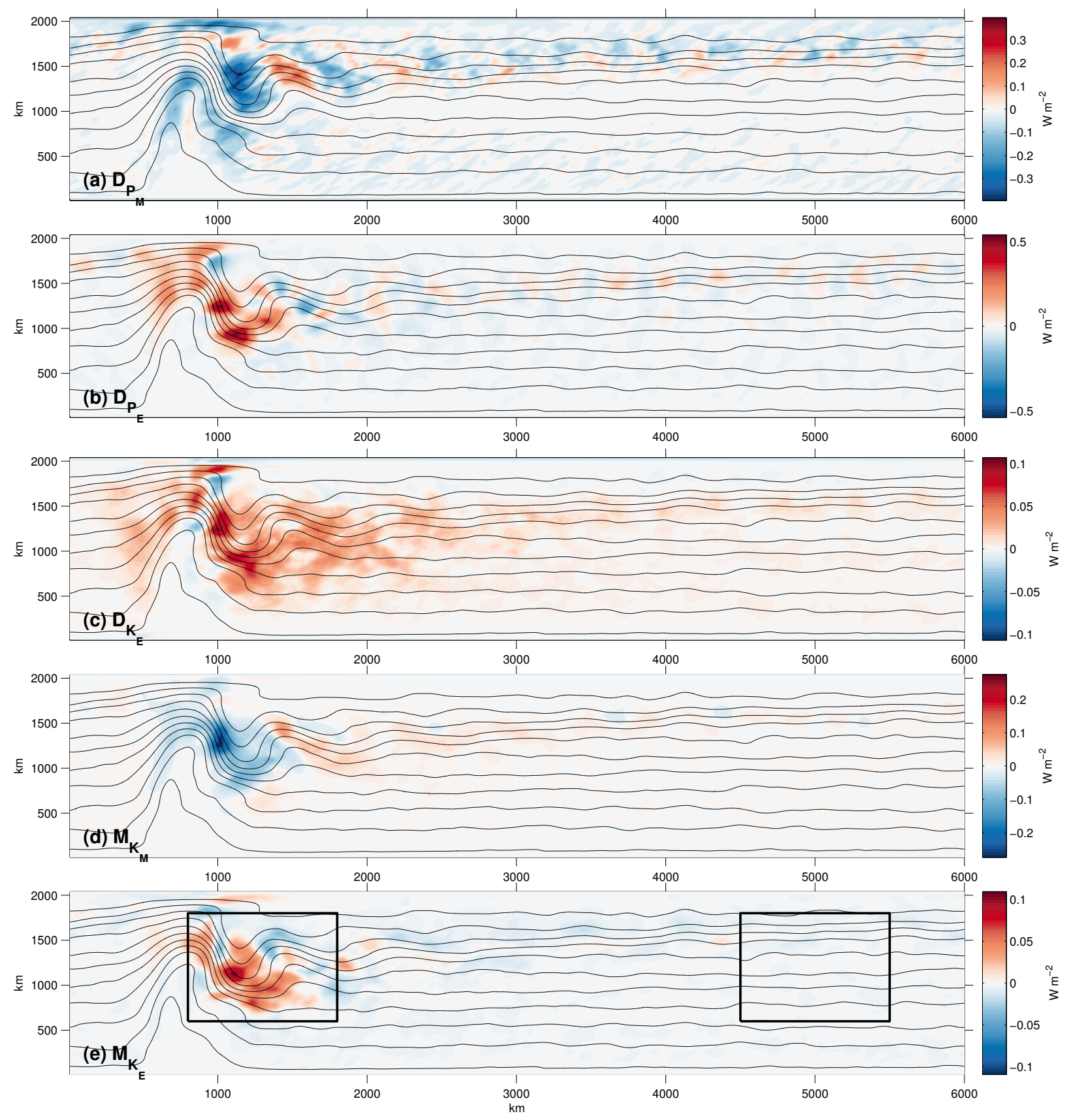

Figure B-3: Depth-integrated energy conversion terms $\left(\mathrm{W} \mathrm{m}^{-2}\right)$ for the experiment with $\tau_{0}=0.1 \mathrm{~N} \mathrm{~m}^{-2}$. Contour lines show temperature every degree Celsius at $177 \mathrm{~m}$ depth. $D_{P_{M}}$ is the rate of change of MAPE due to horizontal eddy fluxes (B.5), $D_{P_{E}}$ is the rate of change of eddy energy due to horizontal eddy density fluxes (B.6), $D_{K_{E}}$ is the rate of change in EKE from EAPE (B.7), $M_{K_{M}}$ is the rate of change in MKE due to eddy momentum fluxes (B.8) and $M_{K_{E}}$ is the rate of change in EKE due to eddy momentum fluxes (B.9). 

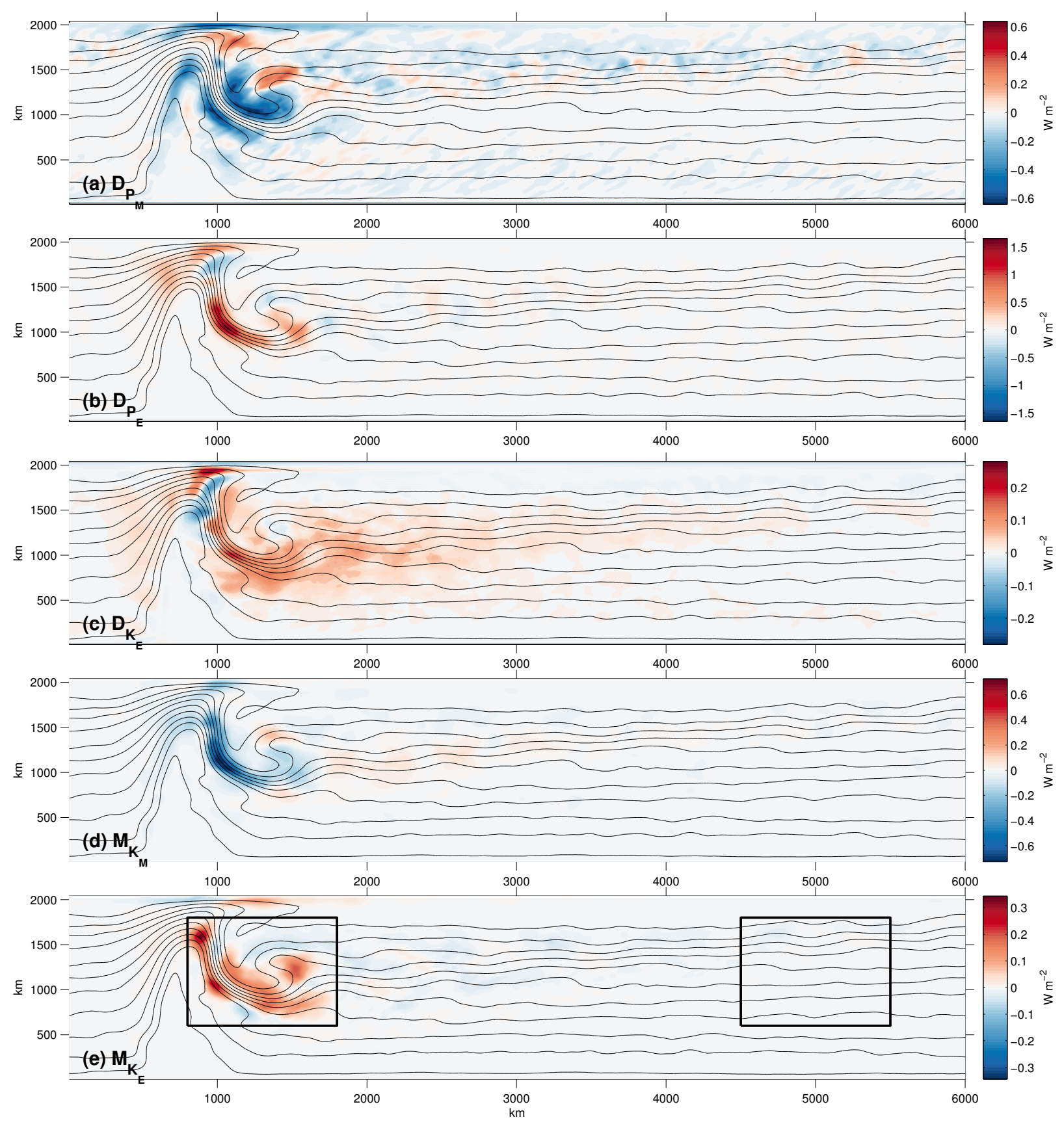

Figure B-4: Depth-integrated energy conversion terms $\left(\mathrm{W} \mathrm{m}^{-2}\right)$ with $\tau_{0}=0.2 \mathrm{~N} \mathrm{~m}^{-2}$. See caption of Fig. B-3 for further details. 


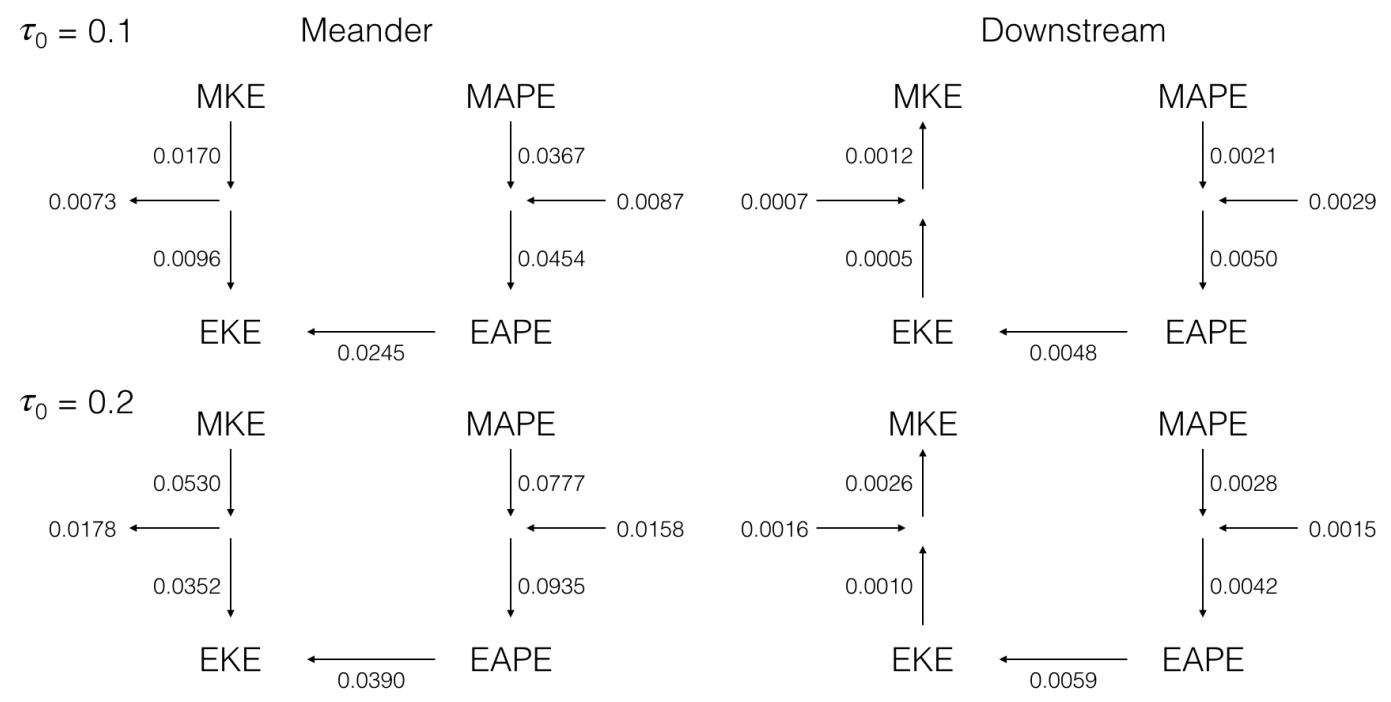

Figure B-5: Lorenz diagram from two different regions, indicated by the boxes in Figs. B-3 and $\mathrm{B}-4$. The meander region is located just downstream of the ridge and the downstream domain refers to a region with quasi-zonal jets. The values provided in the diagram are depth-integrated, domain-averaged quantities; the units are $\mathrm{W} \mathrm{m}^{-2}$. The diagrams follow those shown in Chen et al. 2014. 

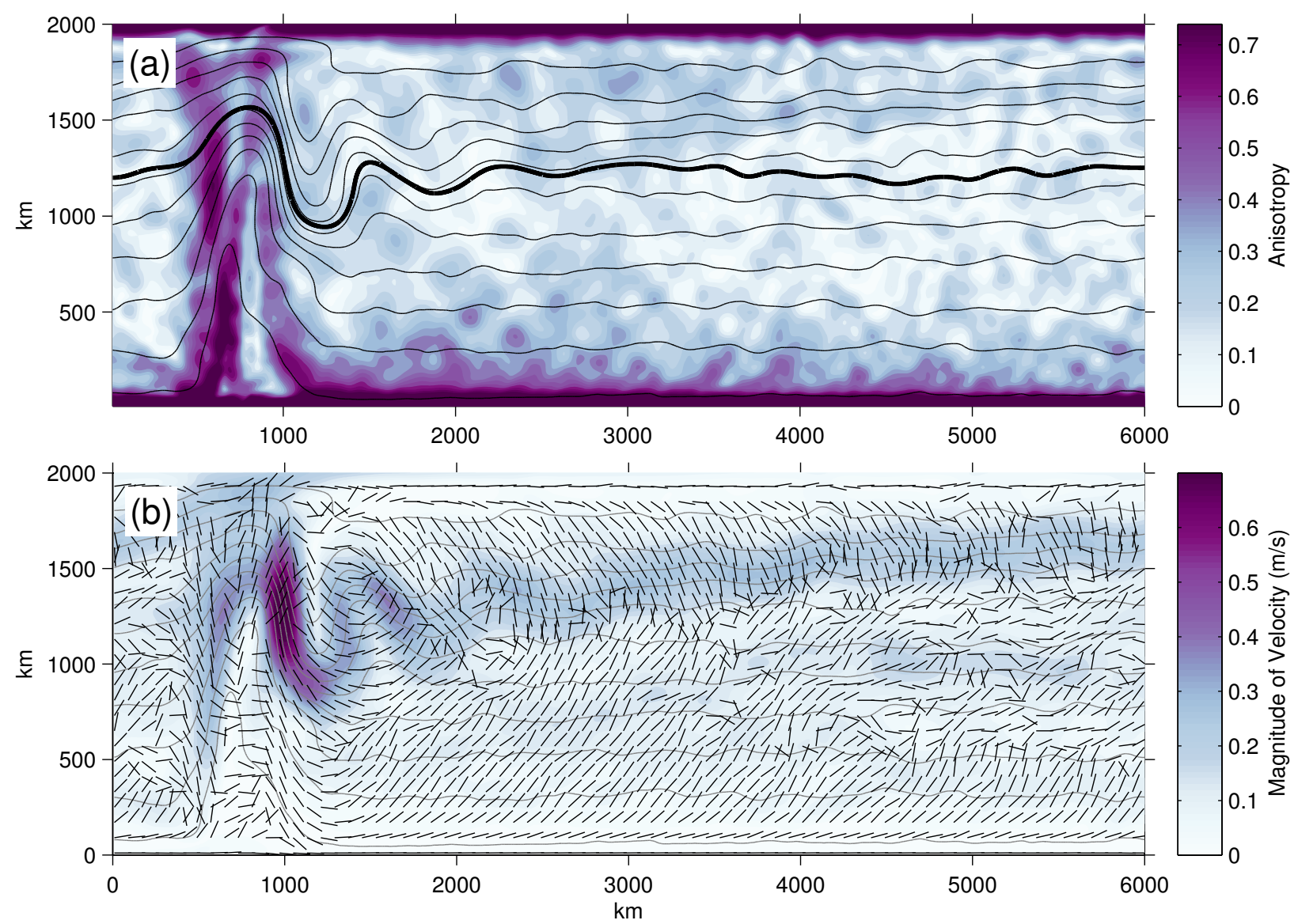

Figure B-6: Depth-averaged eddy anisotropy in color (a) and the tilt (b) for $\tau_{0}=0.1 \mathrm{~N}$ $\mathrm{m}^{-2}$; both diagnostics are dimensionless. The color shows the magnitude of the velocity at $177 \mathrm{~m}$ depth. The temperature is contoured every degree at $177 \mathrm{~m}$ depth and the bold line is a streamline computed from the velocities and is used in Fig. B-8. 


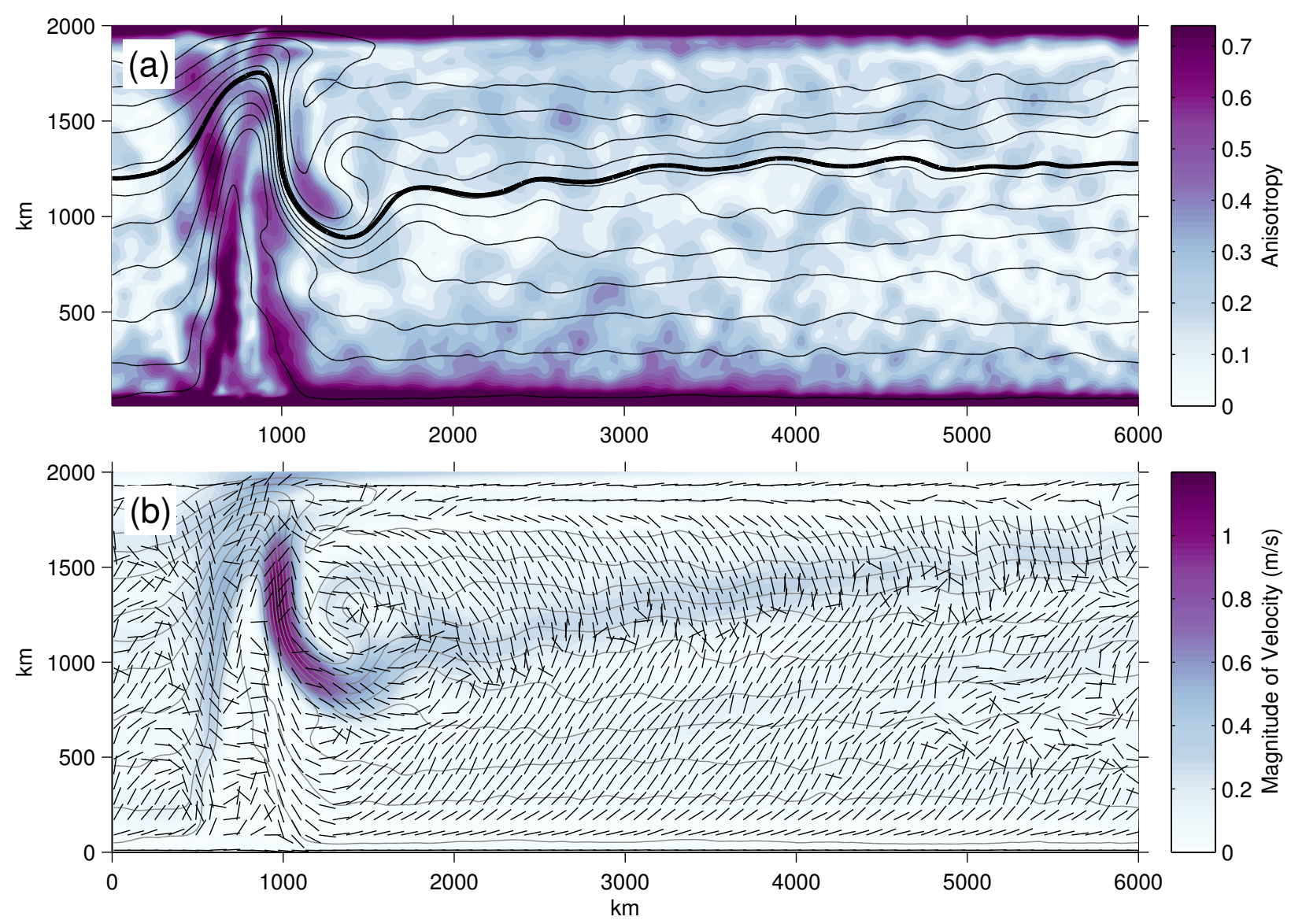

Figure B-7: Depth-averaged eddy anisotropy in color (a) and the tilt (b) for $\tau_{0}=0.2 \mathrm{~N}$ $\mathrm{m}^{-2}$; both diagnostics are dimensionless. The color shows the magnitude of the velocity at $177 \mathrm{~m}$ depth. The temperature is contoured every degree at $177 \mathrm{~m}$ depth and the bold line is a streamline computed from the velocities and is used in Fig. B-9. 

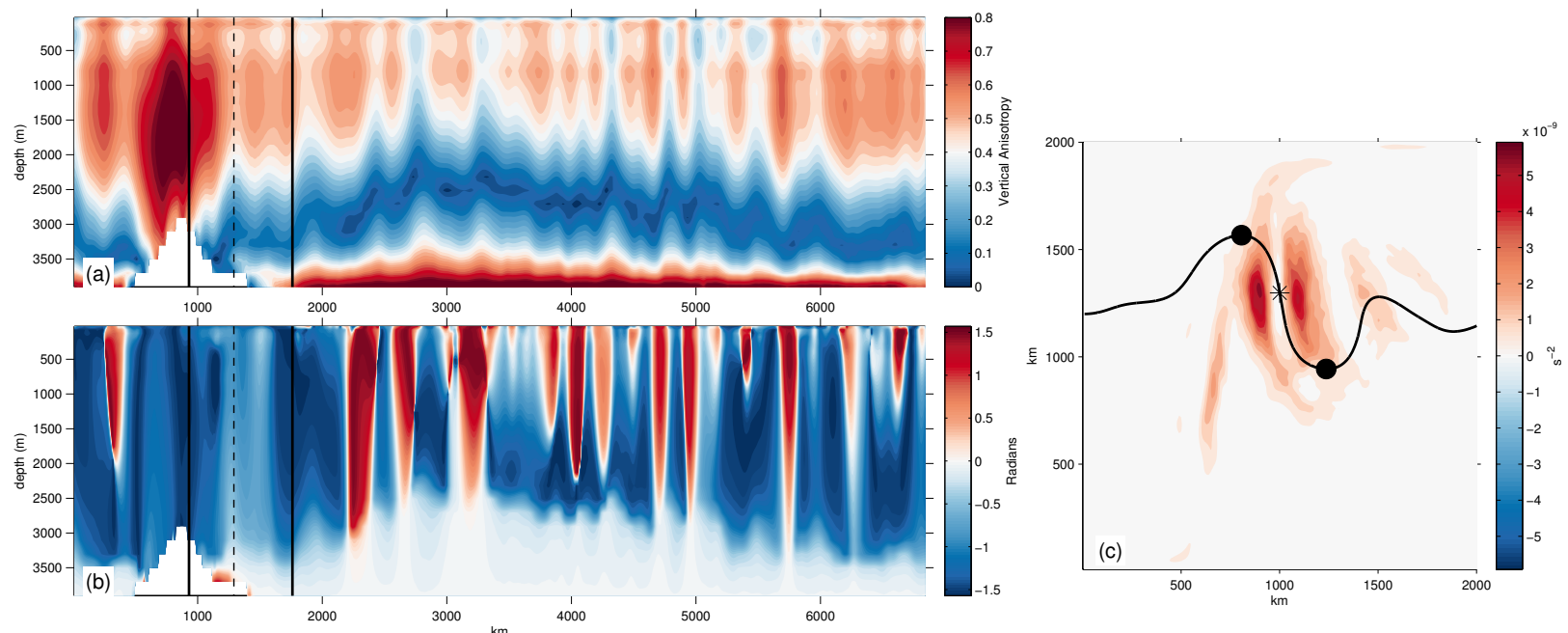

Figure B-8: (a) Vertical eddy anisotropy and (b) the tilt into the vertical with respect to the mean flow (Eq. 16) for $\tau_{0}=0.1 \mathrm{~N} \mathrm{~m}^{-2}$. The calculations are complete along the streamline shown in Fig. B-6. The solid black lines show the location of the $\bullet$ 's in (c) and the dashed line shows the location of the $*$ in (c). A positive tilt (red) represents downgradient buoyancy fluxes, and a negative (blue) tilt represents up-gradient buoyancy fluxes. (c) A measure of the horizontal shear $|\nabla u|^{2}+|\nabla v|^{2}$ with the streamline followed in (a) and (b).
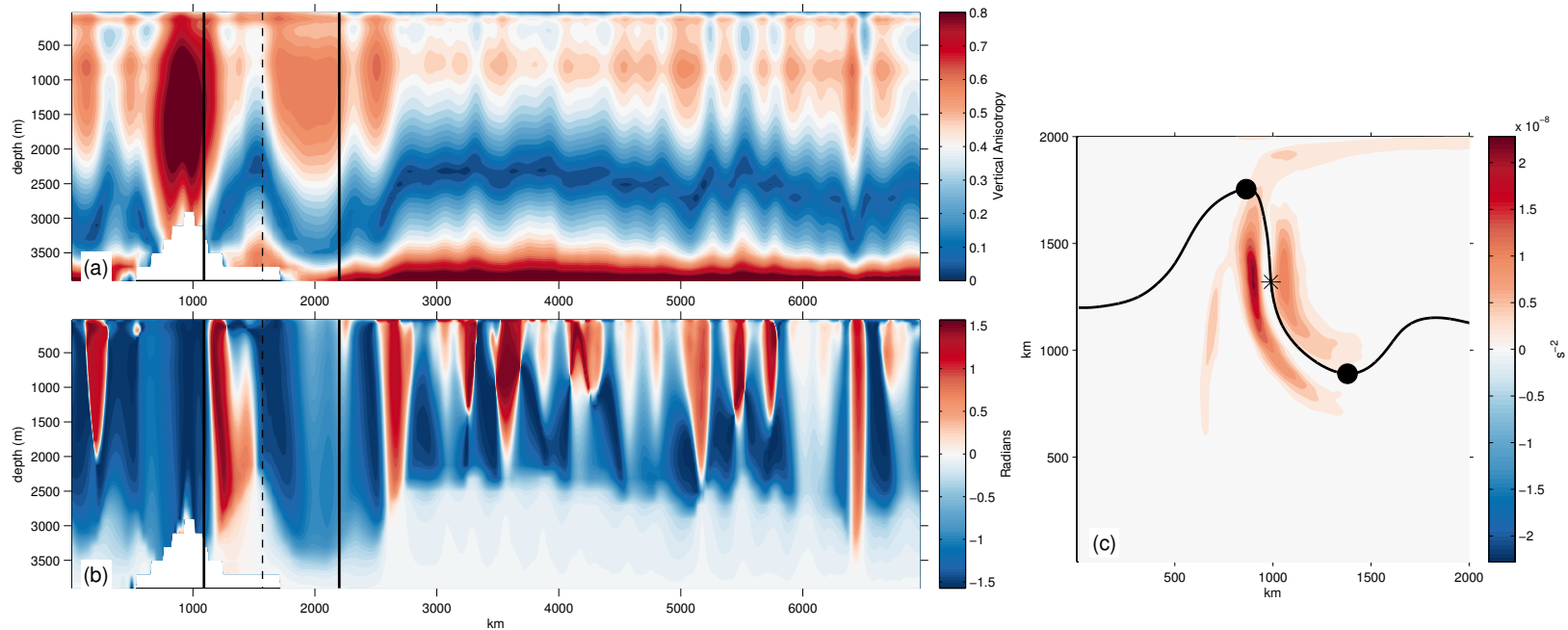

Figure B-9: (a) Vertical eddy anisotropy and (b) the tilt into the vertical with respect to the mean flow (Eq. 16) for $\tau_{0}=0.2 \mathrm{~N} \mathrm{~m}^{-2}$ along the streamline shown in Fig. B-7. Details follow Fig. B-8 

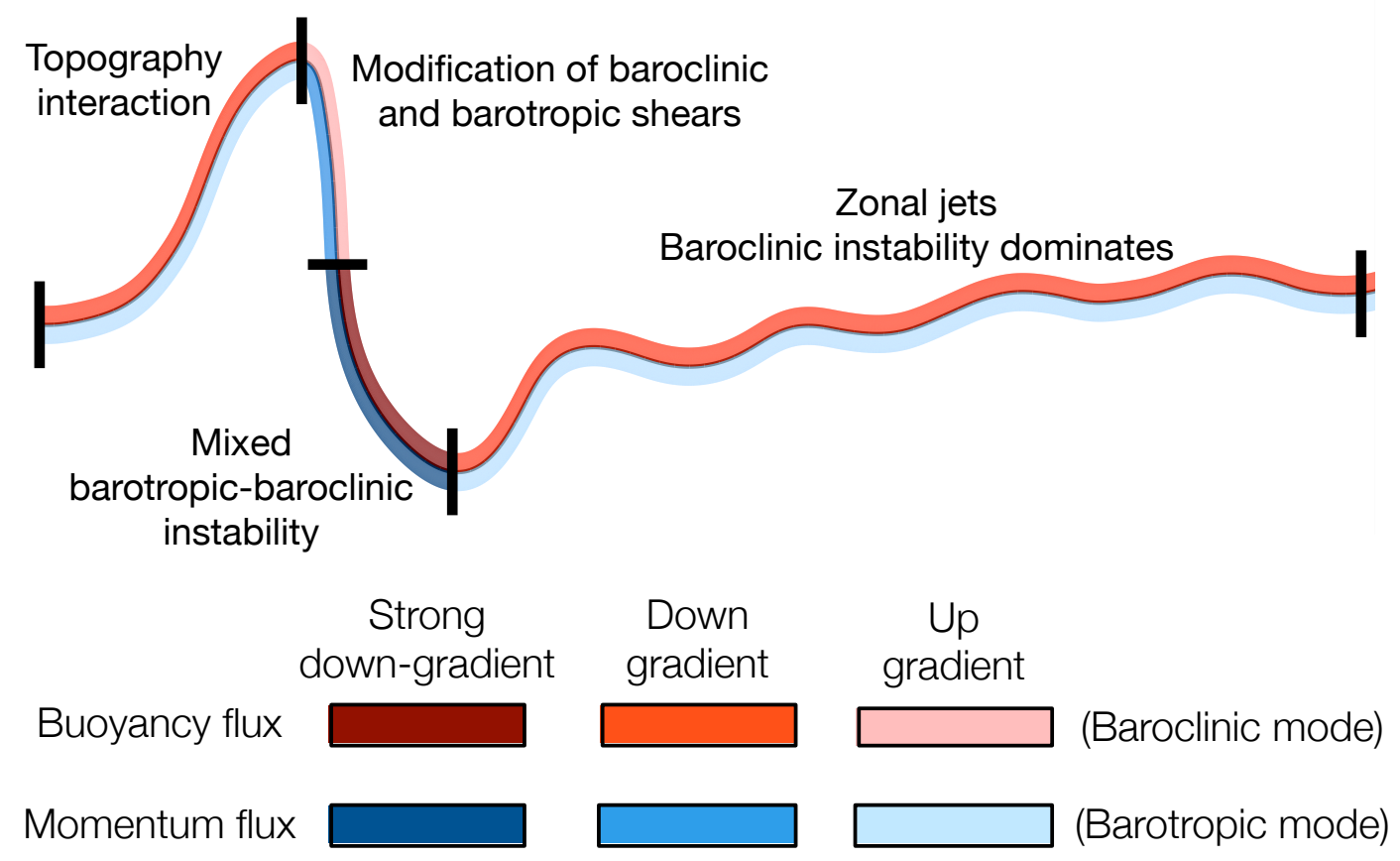

Figure B-10: Schematic summarizing the various regimes in the ACC-like channel; critically there are multiple regimes within the meander region itself. Colors indicate the direction of the eddy buoyancy and momentum fluxes. Upstream of the meander as the flow encounters the topography, the convergence of streamlines both in the horizontal and vertical leads to an increase in eddy anisotropy. Immediately downstream of the ridge crest, the strong rotation of flow direction leads to up-gradient buoyancy fluxes and up- and down-gradient momentum fluxes. The flow becomes barotropically and baroclinically unstable resulting in rapid generation of eddy kinetic energy (EKE). Downstream, zonal jets develop and baroclinic instability dominates with up-gradient momentum fluxes. 


\section{Bibliography}

Abernathey, R. P., and P. Cessi, Topographic Enhancement of Eddy Efficiency in Baroclinic Equilibration, Journal of Physical Oceanography, 44(8), 2107-2126, doi:10.1175/ JPO-D-14-0014.1, 2014.

Abernathey, R. P., J. Marshall, and D. Ferreira, The Dependence of Southern Ocean Meridional Overturning on Wind Stress, Journal of Physical Oceanography, 41(12), 2261-2278, doi:10.1175/JPO-D-11-023.1, 2011.

Ardyna, M., et al., Hydrothermal vents trigger massive phytoplankton blooms in the southern ocean, Nature communications, 10(1), 1-8, 2019.

Barthel, A., A. McC. Hogg, S. Waterman, and S. Keating, Jet-Topography Interactions Affect Energy Pathways to the Deep Southern Ocean, Journal of Physical Oceanography, 47(7), 1799-1816, doi:10.1175/jpo-d-16-0220.1, 2017.

Bischoff, T., and A. F. Thompson, Configuration of a Southern Ocean Storm Track, Journal of Physical Oceanography, 44 (12), 3072-3078, 2014.

Bishop, S. P., P. R. Gent, F. O. Bryan, A. F. Thompson, M. C. Long, and R. P. Abernathey, Southern Ocean Overturning Compensation in an Eddy-Resolving Climate Simulation, Journal of Physical Oceanography, 46 (5), 1575-1592, doi:10.1175/JPO-D-15-0177.1, 2016.

Böning, C. W., A. Dispert, M. Visbeck, S. Rintoul, and F. U. Schwarzkopf, The response of the Antarctic Circumpolar Current to recent climate change, Nature Geoscience, 1(12), 864-869, 2008.

Boyle, E. A., Quaternary deepwater paleoceanography, Science, 249(4971), 863, 1990.

Broecker, W. S., et al., The great ocean conveyor, Oceanography, 4(2), 79-89, 1991.

Chang, E. K. M., S. Lee, and K. L. Swanson, Storm track dynamics, J. Clim., 15, 2163-2183, 2002 .

Chapman, C. C., A. M. Hogg, A. E. Kiss, and S. R. Rintoul, The dynamics of southern ocean storm tracks, Journal of Physical Oceanography, 45(3), 884-903, 2015.

Charney, J. G., and A. Eliassen, A numerical method for predicting the perturbations of the middle latitude westerlies, Tellus, 1(2), 38-54, 1949.

Charney, J. G., and G. R. Flierl, Oceanic analogues of large-scale atmospheric motions, 1981. 
Chelton, D. B., R. A. DeSzoeke, M. G. Schlax, K. El Naggar, and N. Siwertz, Geographical Variability of the First Baroclinic Rossby Radius of Deformation, Journal of Physical Oceanography, 28(3), 433-460, doi:10.1175/1520-0485(1998)028<0433:gvotfb > 2.0.co;2, 1998.

Chen, R., G. R. Flierl, and C. Wunsch, A description of local and nonlocal eddy-mean flow interaction in a global eddy-permitting state estimate, Journal of Physical Oceanography, 44(9), 2336-2352, 2014.

Chen, R., A. F. Thompson, G. R. Flierl, and C. Wunsch, Energy partition and eddy-mean flow interatction in energetic oceanic regions: a time-dependent perspective, Journal of Physical Oceanography, Submitted, 2016.

Constantinou, N. C., A barotropic model of eddy saturation, Journal of Physical Oceanography, 48, doi:10.1175/JPO-D-17-0182.1, 2018.

Constantinou, N. C., and W. R. Young, Beta-plane turbulence above monoscale topography, Journal of Fluid Mechanics, 827, 415-447, doi:10.1017/jfm.2017.482, 2017.

Danabasoglu, G., J. C. Mcwilliams, and P. R. Gent, The Role of Mesoscale Tracer Transports, Science, pp. 1-4, 1994.

de Boer, A., and A. M. Hogg, Control of the glacial carbon budget by topographically induced mixing, Geophysical Research Letters, 2014.

Deacon, G., The hydrology of the southern ocean, 124 pp, Discovery Rep, 15, 1937.

Dijkstra, H. A., Characterization of the multiple equilibria regime in a global ocean model, Tellus A, 59(5), 695-705, 2007.

Downes, S. M., and A. M. Hogg, Southern ocean circulation and eddy compensation in cmip5 models, Journal of Climate, 26(18), 7198-7220, 2013.

Dufour, C. O., et al., Role of Mesoscale Eddies in Cross-Frontal Transport of Heat and Biogeochemical Tracers in the Southern Ocean, Journal of Physical Oceanography, 45(12), 3057-3081, 2015.

Farneti, R., et al., An assessment of Antarctic Circumpolar Current and Southern Ocean meridional overturning circulation during 1958-2007 in a suite of interannual CORE-II simulations, Ocean Modelling, 93, 84-120, doi:10.1016/j.ocemod.2015.07.009, 2015.

Ferrari, R., and M. Nikurashin, Suppression of eddy diffusivity across jets in the Southern Ocean, Journal of Physical Oceanography, 40(7), 1501-1519, 2010.

Ferrari, R., L.-P. Nadeau, D. P. Marshall, L. C. Allison, and H. L. Johnson, A model of the ocean overturning circulation with two closed basins and a reentrant channel, Journal of Physical Oceanography, 47(12), 2887-2906, 2017.

Ferreira, D., J. Marshall, and J.-M. Campin, Localization of deep water formation: Role of atmospheric moisture transport and geometrical constraints on ocean circulation, Journal of Climate, 23(6), 1456-1476, 2010. 
Ferreira, D., et al., Atlantic-Pacific Asymmetry in Deep Water Formation, Annual Review of Earth and Planetary Sciences, 46 (1), 327-352, doi:10.1146/annurev-earth-082517-010045, 2018.

Flierl, G. R., and J. Pedlosky, The Nonlinear Dynamics of Time-Dependent Subcritical Baroclinic Currents, Journal of Physical Oceanography, 37(4), 1001-1021, doi:10.1175/ jpo3034.1, 2007.

Forget, G., and D. Ferreira, Global ocean heat transport dominated by heat export from the tropical pacific, Nature Geoscience, 12(5), 351, 2019.

Forget, G., J.-M. Campin, P. Heimbach, C. Hill, R. Ponte, and C. Wunsch, ECCO version 4: an integrated framework for non-linear inverse modeling and global ocean state estimation, 2015.

Frölicher, T. L., J. L. Sarmiento, D. J. Paynter, J. P. Dunne, J. P. Krasting, and M. Winton, Dominance of the Southern Ocean in anthropogenic carbon and heat uptake in CMIP5 models, Journal of Climate, 28(2), 862-886, doi:10.1175/JCLI-D-14-00117.1, 2015.

Gent, P. R., and J. C. McWilliams, Isopycnal mixing in ocean circulation models, Journal of Physical Oceanography, 20(1), 150-155, 1990.

Gnanadesikan, A., A simple predictive model for the structure of the oceanic pycnocline, Science, 283(5410), 2077-2079, 1999.

Gnanadesikan, A., and R. W. Hallberg, On the Relationship of the Circumpolar Current to Southern Hemisphere Winds in Coarse-Resolution Ocean Models, Journal of Physical Oceanography, 30 (8), 2013-2034, doi:10.1175/1520-0485(2000)030<2013:otrotc > 2.0.co;2, 2000 .

Gnanadesikan, A., R. Kelson, and M. Sten, Flux Correction and Overturning Stability: Insights from a Dynamical Box Model, Journal of Climate, 2018.

Gray, A. R., K. S. Johnson, S. M. Bushinsky, S. C. Riser, J. L. Russell, L. D. Talley, R. Wanninkhof, N. L. Williams, and J. L. Sarmiento, Autonomous Biogeochemical Floats Detect Significant Carbon Dioxide Outgassing in the High-Latitude Southern Ocean, Geophysical Research Letters, 45(17), 9049-9057, doi:10.1029/2018GL078013, 2018.

Gruber, N., P. Landschützer, and N. S. Lovenduski, The variable southern ocean carbon sink, Annual Review of Marine Science, 11(1), 159-186, doi:10.1146/ annurev-marine-121916-063407, pMID: 30212259, 2019.

Gruber, N., et al., Oceanic sources, sinks, and transport of atmospheric co2, Global Biogeochemical Cycles, 23(1), 2009.

Hallberg, R., and A. Gnanadesikan, The role of eddies in determining the structure and response of the wind-driven southern hemisphere overturning: Results from the modeling eddies in the southern ocean (meso) project, Journal of Physical Oceanography, 36(12), $2232-2252,2006$.

Hallberg, R. W., and A. Gnanadesikan, An Exploration of the Role of Transient Eddies in Determining the Transport of a Zonally Reentrant Current, Journal of Physical Oceanography, 31(11), 3312-3330, doi:10.1175/1520-0485(2001)031<3312:AEOTRO > 2.0.CO;2, 2001. 
Haney, R. L., Surface thermal boundary condition for ocean circulation models, Journal of Physical Oceanography, 1(4), 241-248, 1971.

Hogg, A. M., M. P. Meredith, J. R. Blundell, and C. Wilson, Eddy heat flux in the Southern Ocean: Response to variable wind forcing, Journal of Climate, 21(4), 608-620, 2008.

Hogg, A. M., M. P. Meredith, D. P. Chambers, E. P. Abrahamsen, C. W. Hughes, and A. K. Morrison, Recent trends in the Southern Ocean eddy field, Journal of Geophysical Research: Oceans, 120(1), 257-267, 2015a.

Hogg, A. M. C., An Antarctic Circumpolar Current driven by surface buoyancy forcing, Geophysical Research Letters, 37(23), doi:10.1029/2010GL044777, 2010.

Hogg, A. M. C., M. P. Meredith, D. P. Chambers, E. P. Abrahamsen, C. W. Hughes, and A. K. Morrison, Recent trends in the Southern Ocean eddy field, Journal of Geophysical Research: Oceans, 120(1), 257-267, doi:10.1002/2014JC010470, 2015b.

Holmes, R. M., J. D. Zika, R. Ferrari, A. F. Thompson, E. R. Newsom, and M. H. England, Atlantic ocean heat transport enabled by indo-pacific heat uptake and mixing, Geophysical Research Letters, 2019.

Hoskins, B. J., and K. I. Hodges, New perspectives on the Northern Hemisphere winter storm tracks, J. Atmos. Sci., 59, 1041-1061, 2002.

Hoskins, B. J., and P. J. Valdes, On the existence of storm-tracks, J. Atmos. Sci., 47(15), 1854-1864, 1990.

Howard, E., A. McC. Hogg, S. Waterman, and D. P. Marshall, The Injection of Zonal Momentum by Buoyancy Forcing in a Southern Ocean Model, Journal of Physical Oceanography, 45(1), 259-271, doi:10.1175/JPO-D-14-0098.1, 2015.

Ito, T., M. Woloszyn, and M. Mazloff, Anthropogenic carbon dioxide transport in the southern ocean driven by ekman flow, Nature, 463(7277), 80-83, 2010.

James, I., Suppression of baroclinic instability in horizontally sheared flows, Journal of the Atmospheric Sciences, 44(24), 3710-3720, 1987.

James, I., and L. Gray, Concerning the effect of surface drag on the circulation of a baroclinic planetary atmosphere, Quarterly Journal of the Royal Meteorological Society, 112(474), 1231-1250, 1986.

Jansen, M. F., Glacial ocean circulation and stratification explained by reduced atmospheric temperature, Proceedings of the National Academy of Sciences, 114(1), 45-50, 2017.

Jansen, M. F., and L.-P. Nadeau, The Effect of Southern Ocean Surface Buoyancy Loss on the Deep-Ocean Circulation and Stratification, Journal of Physical Oceanography, 46(11), 3455-3470, 2016.

Johnson, G. C., and H. L. Bryden, On the size of the Antarctic Circumpolar Current, Deep-Sea Research, 36(1), 1-15, 1989.

Johnson, H. L., D. P. Marshall, and D. A. Sproson, Reconciling theories of a mechanically driven meridional overturning circulation with thermohaline forcing and multiple equilibria, Climate Dynamics, 29(7-8), 821-836, 2007. 
Jones, C. S., and P. Cessi, Interbasin transport of the meridional overturning circulation, Journal of Physical Oceanography, 46(4), 1157-1169, 2016.

Jones, C. S., and P. Cessi, Size Matters: Another Reason Why the Atlantic Is Saltier than the Pacific, Journal of Physical Oceanography, 47(11), 2843-2859, doi:10.1175/jpo-d-17-0075. $1,2017$.

Karsten, R. H., and J. Marshall, Testing theories of the vertical stratification of the Antarctic Circumpolar Current against observations, Dynamics of Atmospheres and Oceans, 36(13), 233-246, 2002.

Kaspi, Y., and T. Schneider, Downstream self-destruction of storm tracks, Journal of the Atmospheric Sciences, 68(10), 2459-2464, 2011.

Kaspi, Y., and T. Schneider, The role of stationary eddies in shaping midlatitude storm tracks, Journal of the Atmospheric Sciences, 70(8), 2596-2613, 2013.

Key, R. M., et al., Global ocean data analysis project, version 2 (glodapv2), 2015.

Killworth, P. D., Barotropic and baroclinic instability in rotating stratified fluids, Dynamics of Atmospheres and Oceans, 4(3), 143-184, 1980.

Klocker, A., J. R. Maddison, D. P. Marshall, and A. C. Naveira Garabato, Eddy-mean flow interaction in the Antartic Circumpolar Current, Journal of Physical Oceanography, p. submitted, 2016.

Kushner, P. J., and I. M. Held, Potential Vorticity Thickness Fluxes and Wave-Mean Flow Interaction, Journal of the Atmospheric Sciences, 56(7), 948-958, doi:10.1175/ 1520-0469(1999)056<0948:pvtfaw > 2.0.co;2, 2002.

Large, W. G., and S. Pond, Open Ocean Momentum Flux Measurements in Moderate to Strong Winds, Journal of Physical Oceanography, 11(3), 324-336, doi:10.1175/ 1520-0485(1981)011<0324:oomfmi>2.0.co;2, 2002.

Lauderdale, J. M., S. Dutkiewicz, R. G. Williams, and M. J. Follows, Quantifying the drivers of ocean-atmosphere co2 fluxes, Global Biogeochemical Cycles, 30 (7), 983-999, 2016.

Lauvset, S. K., et al., A new global interior ocean mapped climatology: The $1 \times 1$ glodap version 2, Earth System Science Data, 8, 325-340, 2016.

Lin, X., X. Zhai, Z. Wang, and D. R. Munday, Mean, variability, and trend of Southern Ocean wind stress: Role of wind fluctuations, Journal of Climate, 31(9), 3557-3573, doi:10.1175/JCLI-D-17-0481.1, 2018.

Lovenduski, N. S., N. Gruber, S. C. Doney, and I. D. Lima, Enhanced co2 outgassing in the southern ocean from a positive phase of the southern annular mode, Global Biogeochemical Cycles, 21(2), 2007.

Loving, J. L., and G. K. Vallis, Mechanisms for climate variability during glacial and interglacial periods, Paleoceanography, 20(4), 2005.

Lu, J., and K. Speer, Topography, jets, and eddy mixing in the Southern Ocean, Journal of Marine Research, 68(3-4), 479-502, 2010. 
Lumpkin, R., and K. Speer, Global Ocean Meridional Overturning, Journal of Physical Oceanography, 37(10), 2550-2562, doi:10.1175/JPO3130.1, 2007.

MacCready, P., and P. B. Rhines, Meridional Transport across a Zonal Channel: Topographic Localization, Journal of Physical Oceanography, 31(6), 1427-1439, doi:10.1175/ 1520-0485(2001)031<1427:MTAAZC > 2.0.CO;2, 2001.

Maher, P., et al., Model Hierarchies for Understanding Atmospheric Circulation, Reviews of Geophysics, p. 2018RG000607, doi:10.1029/2018RG000607, 2019.

Majkut, J. D., B. R. Carter, T. L. Frölicher, C. O. Dufour, K. B. Rodgers, and J. L. Sarmiento, An observing system simulation for southern ocean carbon dioxide uptake, Philosophical Transactions of the Royal Society A: Mathematical, Physical and Engineering Sciences, 372(2019), 20130,046, 2014.

Mak, J., D. P. Marshall, J. R. Maddison, and S. D. Bachman, Emergent eddy saturation from an energy constrained eddy parameterisation, Ocean Modelling, 112, 125-138, doi: 10.1016/j.ocemod.2017.02.007, 2017.

Mak, J., J. R. Maddison, D. P. Marshall, and D. R. Munday, Implementation of a Geometrically Informed and Energetically Constrained Mesoscale Eddy Parameterization in an Ocean Circulation Model, Journal of Physical Oceanography, 48(10), 2363-2382, doi:10.1175/JPO-D-18-0017.1, 2018.

Marotzke, J., P. Welander, and J. Willebrand, Instability and multiple steady states in a meridional-plane model of the thermohaline circulation, Tellus A: Dynamic Meteorology and Oceanography, 40(2), 162-172, 1988.

Marshall, D., Subduction of water masses in an eddying ocean, Journal of Marine Research, 55(2), 201-222, 1997.

Marshall, D. P., J. R. Maddison, and P. S. Berloff, A framework for parameterizing eddy potential vorticity fluxes, Journal of Physical Oceanography, 42(4), 539-557, 2012.

Marshall, D. P., M. H. Ambaum, J. R. Maddison, D. R. Munday, and L. Novak, Eddy saturation and frictional control of the Antarctic Circumpolar Current, Geophysical Research Letters, 44(1), 286-292, doi:10.1002/2016GL071702, 2017.

Marshall, G. J., Trends in the Southern Annular Mode from observations and reanalyses, Journal of Climate, 16(24), 4134-4143, doi:10.1175/1520-0442(2003)016<4134: TITSAM $>2.0 . \mathrm{CO} ; 2,2003$.

Marshall, J., and T. Radko, Residual-Mean Solutions for the Antarctic Circumpolar Current and Its Associated Overturning Circulation, Journal of Physical Oceanography, 33(11), 2341-2354, doi:10.1175/1520-0485(2003)033<2341:RSFTAC>2.0.CO;2, 2003.

Marshall, J., and G. Shutts, A note on rotational and divergent eddy fluxes, J. Phys. Oceanogr., 11(12), 1677-1680, 1981.

Marshall, J., and K. Speer, Closure of the meridional overturning circulation through Southern Ocean upwelling, Nature Geoscience, 5(3), 171-180, doi:10.1038/ngeo1391, 2012. 
Marshall, J., A. Adcroft, C. Hill, L. Perelman, and C. Heisey, A finite-volume, incompressible Navier Stokes model for studies of the ocean on parallel computers, Journal of Geophysical Research: Oceans, 102(C3), 5753-5766, 1997.

Masich, J., T. K. Chereskin, and M. R. Mazloff, Topographic form stress in the Southern Ocean state estimate, Journal of Geophysical Research: Oceans, 120(12), 7919-7933, 2015 .

McElroy, M., Marine biological controls on atmospheric co2 and climate, Nature, 302(5906), 328-329, 1983.

McWilliams, J. C., W. R. Holland, and J. H. Chow, A description of numerical antarctic circumpolar currents, Dynamics of Atmospheres and Oceans, 2(3), 213-291, doi:10.1016/ 0377-0265(78)90018-0, 1978.

Meredith, M. P., and A. M. C. Hogg, Circumpolar response of Southern Ocean eddy activity to a change in the Southern Annular Mode, Geophysical Research Letters, 33(16), L16,608, doi:10.1029/2006GL026499, 2006.

Meredith, M. P., A. C. Naveira Garabato, A. M. Hogg, and R. Farneti, Sensitivity of the overturning circulation in the southern ocean to decadal changes in wind forcing, Journal of Climate, 25(1), 99-110, 2012.

Morrison, A. K., A. M. C. Hogg, and M. L. Ward, Sensitivity of the Southern Ocean overturning circulation to surface buoyancy forcing, Geophysical Research Letters, 38(14), doi:10.1029/2011GL048031, 2011.

Munday, D. R., and X. Zhai, Sensitivity of Southern Ocean circulation to wind stress changes: Role of relative wind stress, Ocean Modelling, 95, 15-24, doi:10.1016/j.ocemod. 2015.08.004, 2015.

Munday, D. R., H. L. Johnson, and D. P. Marshall, Eddy Saturation of Equilibrated Circumpolar Currents, Journal of Physical Oceanography, 43(3), 507-532, doi:10.1175/ JPO-D-12-095.1, 2013.

Munday, D. R., H. L. Johnson, and D. P. Marshall, The role of ocean gateways in the dynamics and sensitivity to wind stress of the early Antarctic Circumpolar Current, $P a$ leoceanography, 30(3), 284-302, doi:10.1002/2014PA002675, 2015.

Munk, W. H., Abyssal recipes, in Deep Sea Research and Oceanographic Abstracts, vol. 13, pp. 707-730, Elsevier, 1966.

Munk, W. H., and E. Palmén, Note on the Dynamics of the Antarctic Circumpolar Current, Tellus, 3(1), 53-55, doi:10.3402/tellusa.v3i1.8609, 1951.

Nadeau, L.-P., and R. Ferrari, The Role of Closed Gyres in Setting the Zonal Transport of the Antarctic Circumpolar Current, Journal of Physical Oceanography, 45 (6), 1491-1509, doi:10.1175/JPO-D-14-0173.1, 2015.

Nadeau, L.-P., and D. N. Straub, Basin and Channel Contributions to a Model Antarctic Circumpolar Current, Journal of Physical Oceanography, 39(4), 986-1002, doi:10.1175/ 2008jpo4023.1, 2009. 
Nakamura, N., Momentum flux, flow symmetry, and the nonlinear barotropic governor, Journal of the Atmospheric Sciences, 50(14), 2159-2179, 1993.

Naveira Garabato, A. C., R. Ferrari, and K. L. Polzin, Eddy stirring in the Southern Ocean, Journal of Geophysical Research: Oceans (1978-2012), 116 (C9), 2011.

Newsom, E. R., and A. F. Thompson, Reassessing the role of the indo-pacific in the ocean's global overturning circulation, Geophysical Research Letters, 45(22), 12-422, 2018.

Nikurashin, M., and G. Vallis, A theory of deep stratification and overturning circulation in the ocean, Journal of Physical Oceanography, 41(3), 485-502, 2011.

Nikurashin, M., and G. Vallis, A theory of the interhemispheric meridional overturning circulation and associated stratification, Journal of Physical Oceanography, 42(10), 16521667, 2012.

Nilsson, J., P. L. Langen, D. Ferreira, and J. Marshall, Ocean basin geometry and the salinification of the atlantic ocean, Journal of Climate, 26(16), 6163-6184, doi:10.1175/ JCLI-D-12-00358.1, 2013.

Pedlosky, J., Geophysical Fluid Dynamics, New York and Berlin, Springer-Verlag, 1982., doi:10.1007/978-1-4612-4650-3, 1987.

Pedlosky, J., Simple models for local instabilities in zonally inhomogeneous flows, Journal of the Atmospheric Sciences, 46(12), 1769-1778, 1989.

Pedlosky, J., Geophysical Fluid Dynamics, Springer Science \& Business Media, 2013.

Pierrehumbert, R., Local and global baroclinic instability of zonally varying flow, Journal of the Atmospheric Sciences, 41(14), 2141-2162, 1984.

Plumb, R. A., and R. Ferrari, Transformed Eulerian-Mean Theory. Part I: Nonquasigeostrophic Theory for Eddies on a Zonal-Mean Flow, Journal of Physical Oceanography, 35(2), 165-174, doi:10.1175/JPO-2669.1, 2005.

Rahmstorf, S., On the freshwater forcing and transport of the Atlantic thermohaline circulation, Climate Dynamics, 12(12), 799-811, 1996.

Rahmstorf, S., J. E. Box, G. Feulner, M. E. Mann, A. Robinson, S. Rutherford, and E. J. Schaffernicht, Exceptional twentieth-century slowdown in Atlantic Ocean overturning circulation, Nature climate change, 5(5), 475, 2015.

Rivière, P., A. M. Treguier, and P. Klein, Effects of bottom friction on nonlinear equilibration of an oceanic baroclinic jet, Journal of Physical Oceanography, 34(2), 416-432, 2004.

Sallée, J.-B., R. J. Matear, S. R. Rintoul, and A. Lenton, Localized subduction of anthropogenic carbon dioxide in the southern hemisphere oceans, Nature Geoscience, 5(8), 579-584, 2012.

Schneider, T., The General Circulation of the Atmosphere, The Annual Review of Earth and Planetary Science, 10(4), 114-119, doi:10.1080/00221341108985661, 2006.

Sgubin, G., D. Swingedouw, S. Drijfhout, Y. Mary, and A. Bennabi, Abrupt cooling over the North Atlantic in modern climate models, Nature communications, 8, 14,375, 2017. 
Shakespeare, C. J., and A. M. Hogg, An Analytical Model of the Response of the Meridional Overturning Circulation to Changes in Wind and Buoyancy Forcing, Journal of Physical Oceanography, 42(8), 1270-1287, doi:10.1175/JPO-D-11-0198.1, 2012.

Shepherd, T. G., Mean motions induced by baroclinic instability in a jet, Geophys. Astrophys. Fluid Dynamics, 21, 35-72, doi:10.1080/03091928308210120org/10.1080/ $03091928308210120,1983$.

Solodoch, A., A. L. Stewart, and J. C. McWilliams, Baroclinic instability of axially symmetric flow over sloping bathymetry, Journal of Fluid Mechanics, 799, 2016.

Stewart, K., P. Spence, S. Waterman, J. Le Sommer, J.-M. Molines, J. Lilly, and M. England, Anisotropy of eddy variability in the global ocean, Ocean Modelling, 95, 53-65, 2015.

Stocker, T. F., et al., Climate change 2013: The physical science basis, 2013.

Stommel, H., Thermohaline convection with two stable regimes of flow, Tellus, 13(2), 224$230,1961$.

Straub, D. N., On the Transport and Angular Momentum Balance of Channel Models of the Antarctic Circumpolar Current, Journal of Physical Oceanography, 23(4), 776-782, doi:10.1175/1520-0485(1993)023<0776:OTTAAM>2.0.CO;2, 1993.

Talley, L. D., Descriptive Physical Oceanography: An Introduction, Academic press, 2011.

Tamarin, T., J. R. Maddison, E. Heifetz, and D. P. Marshall, A geometric interpretation of eddy reynolds stresses in barotropic ocean jets, Journal of Physical Oceanography, (2016), 2016.

Tamsitt, V., et al., Spiraling pathways of global deep waters to the surface of the southern ocean, Nature communications, 8(1), 1-10, 2017.

Thompson, A. F., and A. C. Naveira Garabato, Equilibration of the Antarctic Circumpolar Current by Standing Meanders, Journal of Physical Oceanography, 44 (7), 1811-1828, doi: 10.1175/JPO-D-13-0163.1, 2014.

Thompson, A. F., and K. J. Richards, Low frequency variability of Southern Ocean jets, J. Geophys. Res., p. in press, 2011.

Thompson, A. F., and J.-B. Sallée, Jets and topography: Jet transitions and the impact on transport in the Antarctic Circumpolar Current, Journal of Physical Oceanography, 42(6), 956-972, 2012.

Thompson, A. F., A. L. Stewart, and T. Bischoff, A multibasin residual-mean model for the global overturning circulation, Journal of Physical Oceanography, 46 (9), 2583-2604, 2016.

Thompson, D. W. J., and S. Solomon, Interpretation of Recent Southern Hemisphere Climate Change, Science, 296(5569), 895-899, doi:10.1126/science.1069270, 2002.

Thornalley, D. J., S. Barker, W. S. Broecker, H. Elderfield, and I. N. McCave, The deglacial evolution of North Atlantic deep convection, Science, 331 (6014), 202-205, 2011. 
Timmermann, A., H. Gildor, M. Schulz, and E. Tziperman, Coherent resonant millennialscale climate oscillations triggered by massive meltwater pulses, Journal of Climate, 16(15), 2569-2585, 2003.

Uchida, T., D. Balwada, R. Abernathey, C. J. Prend, E. Boss, and S. T. Gille, Southern ocean phytoplankton blooms observed by biogeochemical floats, Journal of Geophysical Research: Oceans, 124(11), 7328-7343, 2019.

Vallis, G. K., Atmospheric and Oceanic Fluid Dynamics: Fundamentals and large-scale circulation, Cambridge University Press, 2006.

Walin, G., The thermohaline circulation and the control of ice ages, Palaeogeography, palaeoclimatology, palaeoecology, 50(2-3), 323-332, 1985.

Ward, M. L., and A. M. Hogg, Establishment of momentum balance by form stress in a wind-driven channel, Oc. Mod., 40, 133-146, 2011.

Warren, B. A., Why is no deep water formed in the North Pacific?, Journal of Marine Research, 41(2), 327-347, 1983.

Waterman, S., and B. J. Hoskins, Eddy shape, orientation, propagation, and mean flow feedback in western boundary current jets, Journal of Physical Oceanography, 43(8), 16661690, 2013.

Waterman, S., and J. M. Lilly, Geometric decomposition of eddy feedbacks in barotropic systems, Journal of Physical Oceanography, 45 (4), 1009-1024, 2015.

Waterman, S. N., and S. R. Jayne, Eddy-mean flow interactions in the along-stream development of a western boundary current jet: An idealized model study, Journal of Physical Oceanography, 2011.

Weijer, W., W. P. de Ruijter, H. A. Dijkstra, and P. J. Van Leeuwen, Impact of interbasin exchange on the atlantic overturning circulation, Journal of Physical Oceanography, 29(9), 2266-2284, 1999.

Williams, P. D., P. L. Read, and T. W. Haine, Testing the limits of quasi-geostrophic theory: application to observed laboratory flows outside the quasi-geostrophic regime, Journal of Fluid Mechanics, 649, 187-203, 2010.

Williams, R. G., C. Wilson, and C. W. Hughes, Ocean and atmosphere storm tracks: The role of eddy vorticity forcing, Journal of Physical Oceanography, 37(9), 2267-2289, 2007.

Wolfe, C. L., and P. Cessi, Multiple regimes and low-frequency variability in the quasiadiabatic overturning circulation, Journal of Physical Oceanography, 45(6), 1690-1708, 2015 .

Youngs, M. K., and G. R. Flierl, Three-Dimensional Southern Ocean Overturning, $A G U$ Advances, In prep.

Youngs, M. K., A. F. Thompson, A. Lazar, and K. J. Richards, ACC Meanders, Energy Transfer, and Mixed Barotropic-Baroclinic Instability, Journal of Physical Oceanography, 47(6), 1291-1305, doi:10.1175/JPO-D-16-0160.1, 2017. 
Youngs, M. K., G. Flierl, and R. Ferrari, Role of Residual Overturning for the Sensitivity of Southern Ocean Isopycnal Slopes to Changes in Wind, Journal of Physical Oceanography, 49, 2019. 